\title{
Experimental quantum simulations of many-body physics with trapped ions
}

\author{
Ch Schneider ${ }^{1,2}$, Diego Porras ${ }^{3}$ and Tobias Schaetz ${ }^{1,2}$ \\ ${ }^{1}$ Max-Planck-Institut für Quantenoptik, Hans-Kopfermann-Straße 1, 85748 Garching, Germany \\ ${ }^{2}$ Albert-Ludwigs-Universität Freiburg, Physikalisches Institut, Hermann-Herder-Str. 3, 79104 Freiburg, \\ Germany \\ ${ }^{3}$ Departamento de Física Teórica I, Universidad Complutense, 28040 Madrid, Spain \\ E-mail: tobias.schaetz@mpq.mpg.de
}

Received 24 August 2009, in final form 10 August 2011

Published 17 January 2012

Online at stacks.iop.org/RoPP/75/024401

\begin{abstract}
Direct experimental access to some of the most intriguing quantum phenomena is not granted due to the lack of precise control of the relevant parameters in their naturally intricate environment. Their simulation on conventional computers is impossible, since quantum behaviour arising with superposition states or entanglement is not efficiently translatable into the classical language. However, one could gain deeper insight into complex quantum dynamics by experimentally simulating the quantum behaviour of interest in another quantum system, where the relevant parameters and interactions can be controlled and robust effects detected sufficiently well. Systems of trapped ions provide unique control of both the internal (electronic) and external (motional) degrees of freedom. The mutual Coulomb interaction between the ions allows for large interaction strengths at comparatively large mutual ion distances enabling individual control and readout. Systems of trapped ions therefore exhibit a prominent system in several physical disciplines, for example, quantum information processing or metrology. Here, we will give an overview of different trapping techniques of ions as well as implementations for coherent manipulation of their quantum states and discuss the related theoretical basics. We then report on the experimental and theoretical progress in simulating quantum many-body physics with trapped ions and present current approaches for scaling up to more ions and more-dimensional systems.
\end{abstract}

(Some figures may appear in colour only in the online journal)

\section{Contents}

1. Introduction

2. Tools required for experimental quantum simulations

2.1. Ion traps and Coulomb crystals

2.2. Ions

2.3. Basic operations

2.4. Initialization and readout

3. Theoretical excursion

3.1. Theoretical basics

3.2. $\hat{\sigma}_{x} / \hat{\sigma}_{y}$ interaction

3.3. Effective $\hat{\sigma}_{z} \otimes \hat{\sigma}_{z}$ interaction

3.4. Geometric phase gates

3.5. Quantum Ising Hamiltonian

4. Operations interpreted for experimental quantum simulations
4.1. Simulating the spin

4.2. Simulating the magnetic field

4.3. Simulating the spin-spin interaction

4.4. Geometric phase gate versus adiabatic quantum simulations

5. Towards simulating many-body physics

5.1. Proof-of-principle experiments on quantum spin Hamiltonians

5.2. Systems featuring many-body physics proposed for analogue quantum simulations

6. Scaling analogue quantum simulations in arrays of radio-frequency surface-electrode traps

6.1. One-dimensional radio-frequency

surface-electrode traps 


\subsection{Optimized two-dimensional arrays of radio-frequency surface-electrode traps \\ 6.3. Perspectives of our approach}

7. Scaling quantum simulations based on ions in optical lattices

7.1. Trapping of an ion in a dipole trap

7.2. Lifetime and coherence times of optically trapped ions

7.3. Towards ions and atoms in a common optical lattice

\section{Conclusions}

Acknowledgments

21 Appendix A. Normal modes and frequencies

Appendix B. Transformations of Pauli operators

Appendix C. Transformations of motional operators 26

Appendix D. Matrix elements of displacement operator

Appendix E. System of differential equations of the Rabi problem

Appendix F. Time evolution operator

\section{Introduction}

Simulations and deeper understanding of the dynamics of some tens of interacting spins are already intractable with the most powerful classical computers. For instance, the generic state of 50 spin- $1 / 2$ particles is defined by $2^{50}$ numbers and to describe its evolution a $2^{50} \times 2^{50}$ matrix has to be exponentiated [1]. Recently, one of the ten most powerful supercomputers, JUGENE in Jülich, was exploring this regime. The current record was set by simulating a system of 42 quantum bits (qubits), equivalent to 42 spin-1/2 particles [2, 3].

In any case, it will not help to increase the impressive classical calculation capabilities to simulate only slightly larger quantum systems. Each doubling of the computational power will just allow the addition of one spin/qubit to the system (approximately after two years, according to Moore's law [4]). Furthermore, simply pursuing this path of exponential growth in the computer's classical capabilities would require an exponential shrinking of its electronic components ${ }^{4}$. The structure size currently amounts to approximately $30 \mathrm{~nm}$, a distance spanned by roughly 100 atoms. If gifted engineers further miniaturize the sizes of their structures, 'currents' of a few electrons will 'flow' on 'wires' spanned by a few atoms only. As a consequence, quantum effects will have to be considered for future classical computers, leading to serious consequences. Electrons charging a capacitor, for example, currently realize a storage of logical information: a charged capacitor represents a 'one', a discharged capacitor a 'zero'. What, if the few electrons, classically well caught within the potential of the capacitor, follow their natural quantum mechanical paths and simply escape through the walls by tunnelling?

However, allowing for quantum effects in a controlled way might also be exploited as a feature. Richard Feynman originally proposed [5] using a well controlled quantum system to efficiently track problems that are very hard to address on classical computers and named the device a 'quantum computer' (QC). Nowadays his proposal can be seen closer to the description of a quantum simulator $(\mathrm{QS})^{5}$. In any case, his idea has been theoretically investigated and further developed

\footnotetext{
4 The electronic components are arranged in two dimensions and, only recently, the third dimension is exploited. However, sufficient cooling has to be provided.

5 Depending on the context, the abbreviations 'QC' and 'QS' may also stand for quantum computation and quantum simulation, respectively.
}

to the concept of a universal QC. Fulfilling a well-defined set of prerequisites, known as diVincenzo's criteria [6,7], should make possible running any classical and quantum algorithm by a stroboscopic sequence of operations. These have to act on single qubits, for example, changing their state, and on pairs of qubits performing changes on one qubit, conditional on the state of its mate.

Hundreds of groups worldwide work on many approaches in different fields of atomic, molecular and solid-state systems to realize their version of the envisioned QC. For a concise review see, for example, [8].

However, even assuming an ideal system and perfect operations will require the control of the order of $10^{3}$ logical qubits as a basis for translating any algorithm or the quantum dynamics of a complex system into a sequence of stroboscopic gate operations on a potential universal QC [9]. Residual decoherence will cause computational errors and must be minimized to allow for high operational fidelities ( $~ 99.99 \%-$ 99.9\%) [10]. At present, only then the errors could be overcome by quantum error correction, at the price of a reasonable but still tremendous overhead of ancilla qubits, approximately another 100 per logical qubit. In total, of the order of $10^{5}$ qubits are required. Even though there appear to be no fundamental obstacles for enhancing the fidelities of the operations and for scaling the size of the systems [11], there is still challenging technological development ahead. The realization of a universal QC is not expected within the next decades.

A shortcut via analogue QS has been taken into consideration [5] to allow deeper insight into the dynamics of quantum systems. 'Analogue' emphasizes that the dynamics of the system are not translated into an algorithm of gate operations on subsets of qubits. In contrast, a system of quantum particles is required, where (1) the initial state and its dynamics can be precisely controlled, (2) as many relevant parameters as possible manipulated and (3) the readout of the important characteristics of the final state performed in an efficient way. If the system's evolution was governed by a Hamiltonian suspected to account for the quantum effects of interest, we would be able to experimentally investigate the physics of interest isolated from disturbances, close to Feynman's original proposal. The requirements on the number of quantum particles and fidelities of operations for analogue QS are predicted to be substantially relaxed compared with QC [12]. However, it remains to be investigated which 
realistic assumption on different sources of decoherence in the particular system will lead to a sufficiently small impact on the dedicated QS [13]. It is predicted that QS are less prone to decoherence, for example, in simulating robust effects such as quantum phase transitions (QPT). Therefore, they do not require any precautions in contrast to $\mathrm{QC}$, which suffer from the costly overhead due to quantum error correction. It was even proposed to establish decoherence as an asset [1]. In this context, decoherence is not to be seen as a source of errors, as in the field of universal QC, but as a resource to simulate its natural counterpart. For example, decoherence is suspected to be responsible and required for enhanced efficiencies of (quantum) processes in biological systems at $T \sim 300 \mathrm{~K}$ $[14,15]$.

To discuss the different requirements for different analogue QS, we can distinguish between two categories of simulations. One category deals with problems where QS provide a simulated counterpart that allows intriguing questions that are not directly tractable in the laboratory to be experimentally addressed. Examples are highly relativistic effects such as Hawking and Unruh radiation or the zitterbewegung of a freely moving particle predicted by Dirac's equation (see also [12]). The second category of simulations deals with objectives that are (probably fundamentally) not accessible with classical computation, for example, the complex quantum dynamics of spins in solid-state systems, as mentioned above. A promising strategy is to initialize an analogue QS in a state that can be prepared easily in the system of choice according to step (1) introduced above. Evolving the system adiabatically by changing its parameters according to (2) allows a new state to be reached that is hard or impossible to reach otherwise, for example, via a QPT. The aim here is not to simulate the effects including all disturbances and peculiarities, because the analogue QS would then become as complex as the system to be simulated. The aim can be to investigate whether the simplified model still yields the effects observable in nature and, thereby, to gain a concise deeper understanding of their relevant ingredients. However, there remains room for the important discussion as to whether the specific dynamics emulate nature or simulate the implemented model (Hamiltonian) and whether the results allow the drawing of further conclusions.

In any case, it has to be emphasized that analogue QS are intrinsically not universal. That is, different realizations of a QS will allow the simulation of different systems. Even more important, different approaches for the identical models (Hamiltonians) might allow cross-checking of the validity of the QSs [16].

There are several systems proposed to implement analogue QS, offering different advantages [12] to address the physics in many-body systems. One of them consists of neutral atoms within optical lattices [17-20]. Another promising candidate is based on trapped ions [21-23], originally suggested by Cirac and Zoller in 1995 [24] in the context of QC. Trapped ions already compete at the forefront of many fields, were ultimate accuracy and precision is required, such as metrology (see, for example, [25]). Trapped ions offer unique operational fidelities, individual addressability and short- as well as long-range interactions due to Coulomb forces.

Many models of both categories of QS are promising candidates or already addressed by trapped ions. Examples for the first category are emanating from the fields of cosmology [26-28], relativistic dynamics [29-34], quantum field theory [35], quantum optics [36] including quantum walks as a potential tool for QSs [15, 37-40], chemistry [41], and biology $[42,43]$. For the second category, quantum spin Hamiltonians [21], Bose-Hubbard [22] and spin-boson [44] models were proposed to describe solid-state systems and their simulation would allow the observation and investigation of a rich variety of QPTs [45]. A summary and concise description of theoretical proposals on QS of both categories based on trapped ions and first experimental results up to the year 2008 can be found in [46].

This report aims to describe the current status of the field of experimental, analogue QS addressing many-body physics, its challenges and possible ways to address them. The first proof-of-principle experiment was achieved [47] and extended recently $[48,49]$ on a few trapped ions in linear radio-frequency (RF) traps. The main challenge for QS remains to scale up towards 50-100 ions or even beyond. A simulated system of this size would reach far beyond the regime accessible via classical computation and, even more importantly, allow open scientific questions to be addressed.

The report is organized as follows. In section 2 we introduce the tools available for QS by briefly summarizing the types of traps, different ions species and different technical implementations of the control of the electronic and motional degrees of freedom. In section 3 we derive the mathematical description based on $[21,50,51]$. We aim at extending the existing formalism to be directly applicable to more dimensions and individual trapping conditions envisioned in arrays of ions. We apply this formalism to a basic building block of QC, a two qubit phase gate on the radial modes measured in our group, and emphasize similarities and differences between the application of similar operations for analogue QS. This section is supplemented by a detailed appendix. In section 4, we interpret the interactions in the context of analogue QS, which should be sufficient for understanding the subsequent discussion of the experimental implementations without going through the details of section 3 . In section 5, we first depict the proof-of-principle experiments on a few trapped ions in linear RF traps. Based on the stateof-the-art capabilities we present in the second part of this section a summary of proposals to study many-body physics in a variety of solid-state systems. The two following sections are dedicated to two proposals aiming for scaling up the systems. In section 6 , we discuss potential realizations of a two-dimensional array of RF surface-electrode traps. They are conceptually similar to promising approaches in Penning traps $[50,52,53]$. We will also introduce an alternative approach based on ions in optical traps in section 7, thus, trying to combine the advantages of trapped ions and optical lattices. Finally, we conclude in section 8. 
(a)

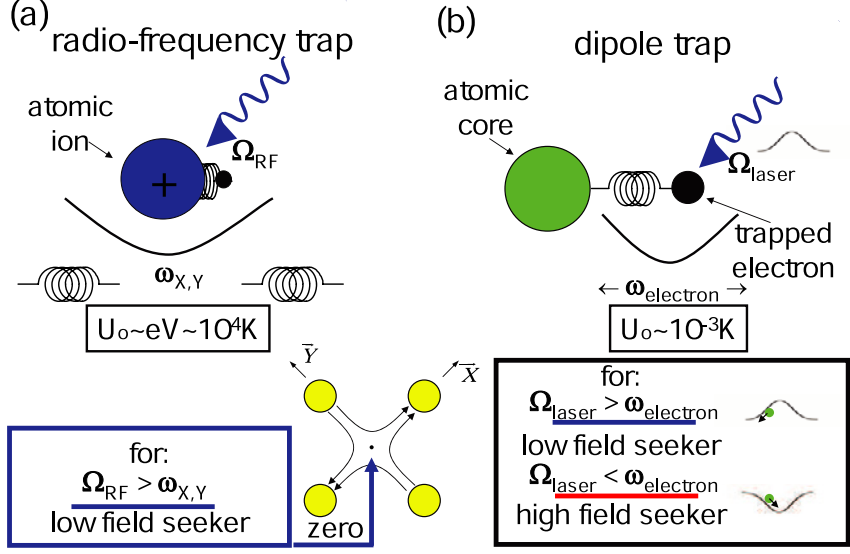

Figure 1. Two concepts for trapping charged particles. Both concepts require electromagnetic fields (blue sinusoidal arrow). (a) In RF traps, an RF field at frequency $\Omega_{\mathrm{RF}} /(2 \pi)$ applied to quadrupole electrodes (yellow circles) interacts with a charged atom directly. The time averaged confining pseudopotential allows the ion to oscillate at frequencies $\omega_{X / Y} /(2 \pi)$ approximately an order of magnitude smaller than $\Omega_{\mathrm{RF}} /(2 \pi)$. Since $\Omega_{\mathrm{RF}}>\omega_{X / Y}$, the trapping field can be understood as blue-detuned with respect to the 'resonance' frequency $\omega_{X / Y}$, consequently the ion will seek the field minimum in the centre of the quadrupole (field lines indicated by black arrows). Typical depths of the pseudopotential are of the order of $k_{\mathrm{B}} \times 10^{4} \mathrm{~K}$. (b) In optical traps, the optical field is typically applied via laser beams that provide an intensity dependent ac Stark shift of the electronic levels of the atom or ion. The frequency $\Omega_{\text {laser }}$ of the laser can be detuned blue (red) with respect to the relevant electronic resonance frequency $\omega_{\text {electron }}$ and therefore forces the atom/ion to seek low (high) fields. Typical depths of the pseudopotential are of the order of $k_{\mathrm{B}} \times 10^{-3} \mathrm{~K}$.

\section{Tools required for experimental quantum simulations}

In this section, we describe the requirements to implement analogue QS based on trapped ions. Most of these tools have been developed over the last decades, many for the purpose of quantum information processing (QIP) with the main focus on QC.

\subsection{Ion traps and Coulomb crystals}

Isolating and trapping of individual particles as well as the precise control of their motional (external) degrees of freedom is key for many high precision measurements. Several trapping concepts have been developed for and implemented with ions, such as RF traps [54], Penning traps [55] and optical traps [56]. The physics of these devices, for example, of RF traps and optical dipole traps, is closely related. Electromagnetic multipole fields act on the charge or induce electric dipole moments. The resulting forces on the particles lead in time average to a confining pseudopotential. The two concepts are compared in figure 1 .

However, there was a delay of more than a decade between trapping charged atoms in RF fields $[57,58]$ and trapping neutral particles with optical fields [59]. One explanation is that RF traps provide potential depths of the order of several $\mathrm{eV} \approx k_{\mathrm{B}} \times 10^{4} \mathrm{~K}$, while optical traps typically store particles up to $k_{\mathrm{B}} \times 10^{-3} \mathrm{~K}$ only. This discrepancy is mainly due to the (a) $>$

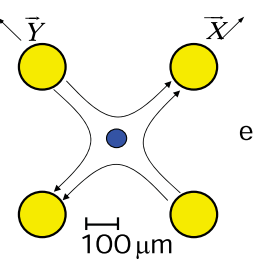

(b)

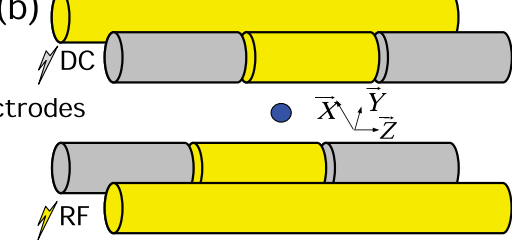

Figure 2. Schematic of the three-dimensional electrode geometry of a linear RF trap. (a) Cross section through the central quadrupole electrodes (yellow) providing the radial confinement for the ion (blue disk). (b) Side view, where segments (grey) are used to apply dc voltages providing a static potential well along the $Z$-axis. Combined with the radial $(X, Y)$ pseudopotential due to the RF field, a three-dimensional confinement is achieved. The ion is stored in ultra-high vacuum and is well protected against disturbances from the environment. However, the fairly open geometry allows access to the external (motional) and internal (electronic) degrees of freedom, for example, with focused laser beams.

comparatively large Coulomb force that $\mathrm{RF}$ fields can exert on charges. The RF field at typical frequencies $\Omega_{\mathrm{RF}} /(2 \pi)=$ $10 \mathrm{MHz}-100 \mathrm{MHz}$ directly acts on the massive ion. The related motional frequencies within the deep pseudopotential amount to a few MHz. Optical fields, in contrast, oscillate more than six orders of magnitude faster: too fast for the massive atomic core to follow. In a simplified picture, the optical field has to induce a dipole moment of the electron and the atomic core first to allow for a subsequent interaction of the dipole with the optical field. Similar to RF traps, the optical field results in a pseudopotential, which is close to identical for neutral atoms and charged ions [60].

Here we focus first on ions in linear RF traps. The concept for the radial confinement is depicted in figures $1(a)$ and 2. The RF field applied to two opposing electrodes of the quadrupole can provide a radially confining pseudopotential. Similar to a quadrupole mass filter, one can find voltages for given parameters (electrode geometry and mass/charge ratio of the ion species) that allow for stable confinement in two dimensions. Additional dc voltages add a static harmonic potential to complete the three-dimensional confinement that can be assumed to be harmonic. Dependent on the application, these dc voltages can be applied to electrodes realized as rings or needles along the axis or by a segmentation of the quadrupole electrodes (see figure $2(b)$ ). A confined ion will oscillate with frequency $\omega_{Z} /(2 \pi)$ along the trap axis and with frequencies $\omega_{X / Y} /(2 \pi)$ in the radial directions. The radial oscillation is superimposed by a fast oscillation at frequency $\Omega_{\mathrm{RF}} /(2 \pi)$ (so-called micromotion), which increases with increasing distance of the ion from the trap centre, such that the RF field does not vanish anymore.

Typical parameters for conventional setups are a minimal ion-electrode distance $h \sim 100 \mu \mathrm{m}-1000 \mu \mathrm{m}$ allowing for RF voltages of the order of $1000 \mathrm{~V}$.

Different laser cooling schemes can be applied to reduce the total energy of motion of the ion [51]. Doppler cooling [61-64] of several ions already allows a regime to be entered, where the kinetic energy $\left(k_{\mathrm{B}} T \sim \mathrm{mK}\right)$ of the ions becomes significantly smaller than the energy related to the mutual Coulomb repulsion. Hence, the ions cannot exchange their position anymore. A phase transition from the gaseous (liquid) 


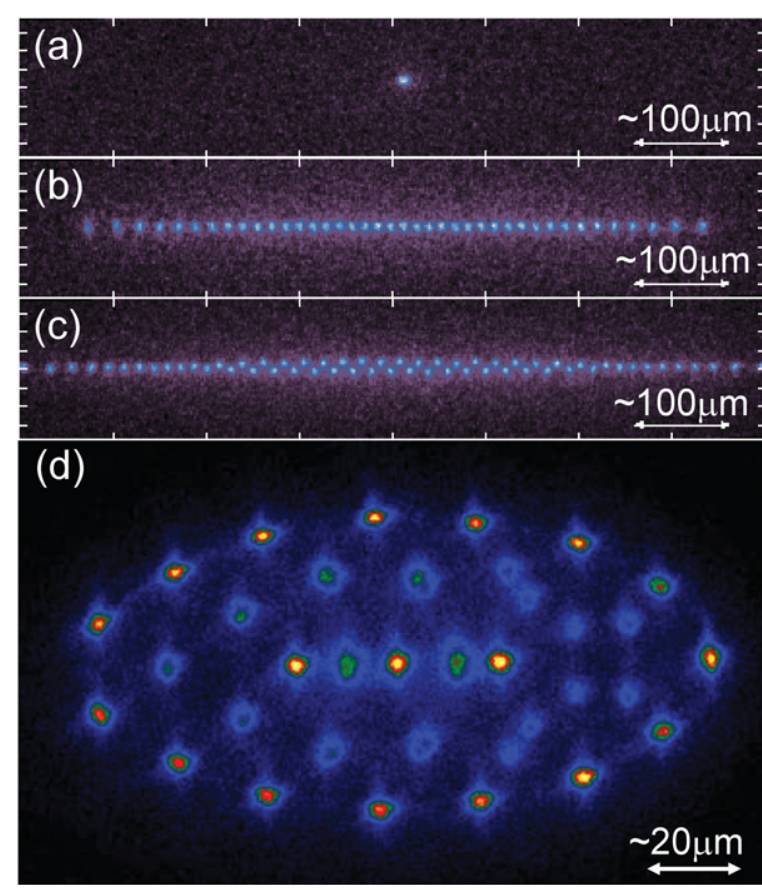

Figure 3. Fluorescence images of laser-cooled ions in a common confining potential of a linear RF trap (see figure 2), forming differently structured Coulomb crystals. (a) A single ion $\left(\mathrm{Mg}^{+}\right)$. (b) A linear chain of 40 ions at $\omega_{X / Y} \gg \omega_{Z}$. The axis of the chain coincides with the trap $Z$-axis, which is identically orientated in the rest of the images. $(c)$ A linear chain embedding a two-dimensional zigzag structure of 60 ions for $\omega_{X / Y}>\omega_{Z}$. (d) A three-dimensional structure of more than 40 ions at $\omega_{X / Y} \gtrsim \omega_{Z}$. The enhanced signal-to-noise ratio in $(d)$ is achieved by extended exposure. Structural phase transitions can be induced between one-, two- and three-dimensional crystals, for example by reducing the ratio of radial to axial trapping frequencies.

plasma to a crystalline structure occurs $[65,66]$. On the one hand, the resulting Coulomb crystals (see figure 3 ) provide many similarities with solid state crystals already partially explaining why Coulomb crystals appear naturally suited to simulate many-body physics. (1) The ions reside on individual lattice sites. (2) The motion of the ions (external degree of freedom) can be described easiest in terms of common motional modes with the related quanta being phonons. The phonons in Coulomb crystals allow long-range interactions to be mediated between the spins associated with the ions. In a different context, the phonons can also be interpreted as bosonic particles, for example, capable of tunnelling between lattice sites simulated by the ions (see also section 5.2). On the other hand, there are advantageous differences compared with solid-state crystals. (3) Coulomb crystals typically build up in ultra-high vacuum $\left(\left(10^{-9}-10^{-11}\right)\right.$ mbar $)$ and are very well shielded against disturbances from the environment, thus providing long coherence times. (4) Coulomb crystals feature lattice constants of a few micrometres (see figure 3 ), dependent on the trapping potential counteracting the mutual Coulomb repulsion. Compared with a solid, where distances are of the order of Angstroms $\left(10^{-10} \mathrm{~m}\right)$ the density of the structure in one dimension is reduced by five, in three dimensions by fifteen orders of magnitude. This allows for individual addressing of the ions and for individual preparation, control and readout of their electronic and motional states. (5) The Coulomb interaction between the charged ions is not shielded within the crystal, as in Coulomb crystals the charge of all ions has the same sign in contrast to ionic crystals in solid-state systems. However, it should be mentioned that, as opposed to quantum solids, the quantum statistics of the ions is not relevant due to the suppression of the mutual exchange processes.

It has to be pointed out that it is possible to deterministically achieve phase transitions between different structures of Coulomb crystals for large numbers of ions $[65,66]$. When the ratio of radial to axial confinement is reduced or the number of confined ions is increased, we observe the transition from a linear chain of ions via a twodimensional zigzag structure to a three-dimensional structure (see figures 3(b)-(d)).

Despite the unique conditions in Coulomb crystals in linear RF traps and the high fidelities of operations, current experimental approaches to QS (and QC) are still limited to a small number of ions. The approaches include of the order of ten ions arranged in a linear chain [49,67]. This is accomplished by choosing the radial confinement much stronger than the axial one. The linear chain orientates along the weakest $(Z)$ direction, where tiny oscillations of the cooled ions around the minimum of the pseudopotential $(X$ and $Y$ ) and thus micromotion still remains negligible.

For the purposes of a QC and QS, scaling to a larger number of spins and more dimensions while keeping sufficient control over all required degrees of freedom remains the challenge of the research field. Using longer linear chains confined in anharmonic axial potentials [68] might provide a way to reach a number of ions in the system that in principle already exceeds capabilities of a classical supercomputer. Another way might be the use of RF ring traps offering periodic boundary conditions for static Coulomb crystals $[65,66]$ and even (more-dimensional) crystalline beams of ions [69-71]. A microfabricated ring trap is currently being developed and fabricated at Sandia National Laboratories [72].

The two main limitations for further scaling of the number of ions in a common potential, from a practical point of view, are (1) the emergence of $3 N$ normal modes for $N$ ions plus their sum and difference frequencies that lead to an increasingly crowded phonon spectrum (already for each spatial dimension separately). Individual spectral components become difficult to identify and off-resonant couplings to 'spectator' transitions [50] are hard to avoid. However, under certain conditions, QSs are predicted to allow for coupling to all modes simultaneously, see for example [21]. (2) QSs based on ions in large, more-dimensional Coulomb crystals suffer from additional challenges, for example, intrinsic micromotion (due to the displacement from the minimum of the pseudopotential), an inhomogeneous ion spacing (due to space charge effects) and the coupling between modes of all three spatial dimensions.

One approach for scalability might be to generate a spinoff from the QIP community based on their new concept of a surface-electrode geometry for RF traps [73,74] (see figure 14). Currently, this design is tested with the aim to allow for networks of interconnected linear traps. This constitutes a promising possibility to realize the multiplex architecture of 
memory and processor traps for universal QC [75]. However, for QS we need a miniaturized array of traps allowing for moredimensional interactions, as discussed in section 6 .

It has to be emphasized that there are other concepts for trapping ions, a prominent one being Penning traps. Penning traps provide trapping potentials of similar parameters as RF traps. A strong, static magnetic field and a dc electric field yield a stable confinement of large, rotating Coulomb crystals. Storing many cool ions in a Penning trap, naturally provides, for example, a large triangular lattice of ions [76-78] that is also predicted to be well suited for QS [79]. Promising results are on their way [52,53]. Another challenging proposal for QS involves trapping ions optionally simultaneously with atoms in optical lattices [80] (see section 7).

\subsection{Ions}

A large variety of different atomic ions have already been used for the purpose of QIP. Every ion or, more specifically, every isotope has different properties, for example, regarding the level scheme or the charge-mass ratio, and thus can meet different requirements of a QS. However, they all have a single valence electron leading to an alkali-like level scheme. Most prominent are the earth alkali ions $\mathrm{Be}^{+}, \mathrm{Mg}^{+}, \mathrm{Ca}^{+}, \mathrm{Sr}^{+}$and $\mathrm{Ba}^{+}$. A similar electronic structure has $\mathrm{Zn}^{+}, \mathrm{Cd}^{+}$and $\mathrm{Hg}^{+}$, followed by $\mathrm{Yb}^{+}[81]$.

Typically, two electronic levels with sufficiently long coherence times are chosen as qubit or spin states $|\downarrow\rangle$ and $|\uparrow\rangle$, respectively. (In principle, however, the restriction to two states is not required and the use of up to 60 states has been proposed for (neutral) holmium [82].) The types of qubits can be divided into two classes: in optical qubits, the states are encoded in two states with a dipole-forbidden transition at an optical frequency. An example is ${ }^{40} \mathrm{Ca}^{+}$with $|\downarrow\rangle:=\left|S_{1 / 2}\right\rangle$ and $|\uparrow\rangle:=\left|D_{5 / 2}\right\rangle$. The lifetime of $|\uparrow\rangle$ is on the order of $1 \mathrm{~s}$, which defines the upper bound for its coherence time. In hyperfine/Zeeman qubits, two sublevels from the ground state manifold are chosen as $|\downarrow\rangle$ and $|\uparrow\rangle$. An applied magnetic field lifts the degeneracy within the manifolds of electronic levels to allow for spectrally resolving the dedicated states. The states of hyperfine/Zeeman qubits have extremely long lifetimes and coherence times on the order of minutes have been observed $[83,84]$. As an example for a hyperfine/Zeeman qubit, an excerpt of the level scheme of ${ }^{25} \mathrm{Mg}^{+}$is shown in figure 4. The transition frequencies in hyperfine/Zeeman qubits are in the microwave regime.

\subsection{Basic operations}

The quantized oscillation of the ions in the harmonically approximated potential of the trap gives rise to motional states, which are typically expressed in terms of Fock states $|n\rangle$. Independent of the choice of qubit we will require three different types of couplings to electronic states and/or motional states to assemble the toolbox for QC and QS based on trapped ions (for details see section 3).

(a) Coupling of the electronic states only $(|\downarrow\rangle|n\rangle \rightleftarrows|\uparrow\rangle|n\rangle)$. This operation can be used to implement Rabi flops between the electronic states and serves as a one-qubit gate

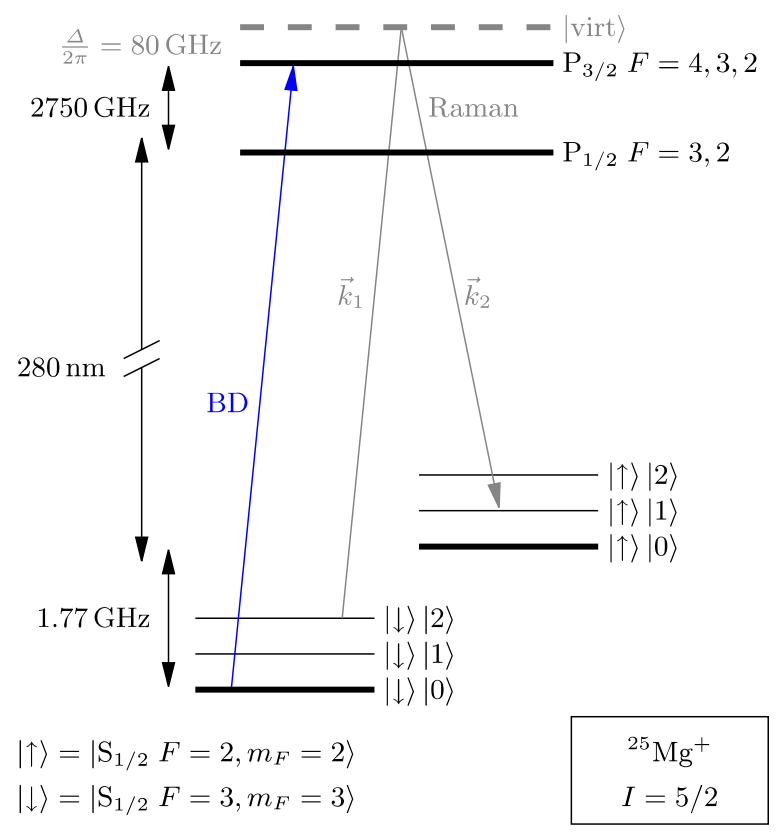

Figure 4. Excerpt of the level scheme of ${ }^{25} \mathrm{Mg}^{+}$as an example of a hyperfine qubit (not to scale). ${ }^{25} \mathrm{Mg}^{+}$has a nuclear spin of $I=5 / 2$ and thus a hyperfine-split ground state $\left(\mathrm{S}_{1 / 2}, F=3\right.$ and $\mathrm{S}_{1 / 2}$, $F=2$ ). By applying a static magnetic field of a few Gauss, the degeneracy of the Zeeman sublevels is lifted. The Doppler cooling laser (labelled 'BD') is $\sigma^{+}$polarized and detuned red by

$\Gamma / 2 \approx 2 \pi \times 20 \mathrm{MHz}$ from the cycling transition $\mathrm{S}_{1 / 2}, F=3$,

$M_{F}=3 \leftrightarrow \mathrm{P}_{3 / 2}, F=4, M_{F}=4$. Here, $\Gamma$ denotes the linewidth of the P levels. The level $|\downarrow\rangle:=\left|\mathrm{S}_{1 / 2}, F=3, M_{F}=3\right\rangle$ and the level $|\uparrow\rangle:=\left|\mathrm{S}_{1 / 2}, F=2, M_{F}=2\right\rangle$ are chosen as qubit states or (simulated) spin states, respectively. The ion is optically pumped into $|\downarrow\rangle$ during cooling. The electronic state is read out by a variant of 'BD', which is resonant on the cycling transition. Hence, an ion in the state $|\downarrow\rangle$ will fluoresce, while an ion in state $|\uparrow\rangle$ is off-resonant by almost $50 \Gamma$ and will remain dark. The motional states of one of the motional modes are indicated as 'ladders' on top of the electronic states. Two laser beams (labelled 'Raman') detuned by $\Delta$ from the $\mathrm{P}_{3 / 2}$ level can be used to drive two-photon stimulated-Raman transitions between $|\downarrow\rangle$ and $|\uparrow\rangle$. A flop on the first red sideband is indicated by the arrows from $|\downarrow\rangle|2\rangle \rightarrow|\uparrow\rangle|1\rangle$.

of a potential QC. In the context of QS it can be interpreted as simulated magnetic field (see also section 3.2).

(b) Coupling of the electronic and motional states $(|\downarrow\rangle|n\rangle \rightleftarrows$ $\left.|\uparrow\rangle\rangle\left|n^{\prime}\right\rangle\right)$. This operation can drive Rabi flops between electronic states and different motional states, for example on the first red $\left(n^{\prime}=n-1\right)$ or blue sideband $\left(n^{\prime}=n+1\right)$ (see also section 3.2). It can be used to create entanglement between the electronic and motional states and is an important ingredient for both sideband cooling and the readout of the motional state (see below).

(c) State-dependent forces (for example, $|\downarrow\rangle|n\rangle \rightarrow$ $|\downarrow\rangle|n+1\rangle)$. These forces lead to state-dependent displacements. They can be used for conditional interactions between multiple ions, which are exploited for quantum gates (see sections 3.3 and 3.4) or effective spin-spin interactions in the simulation of quantum spin Hamiltonians (see section 3.5).

Operations (a)-(c) can be realized for both classes of qubits in the optical regime and for hyperfine/Zeeman qubits additionally via microwave fields $[50,51]$. 

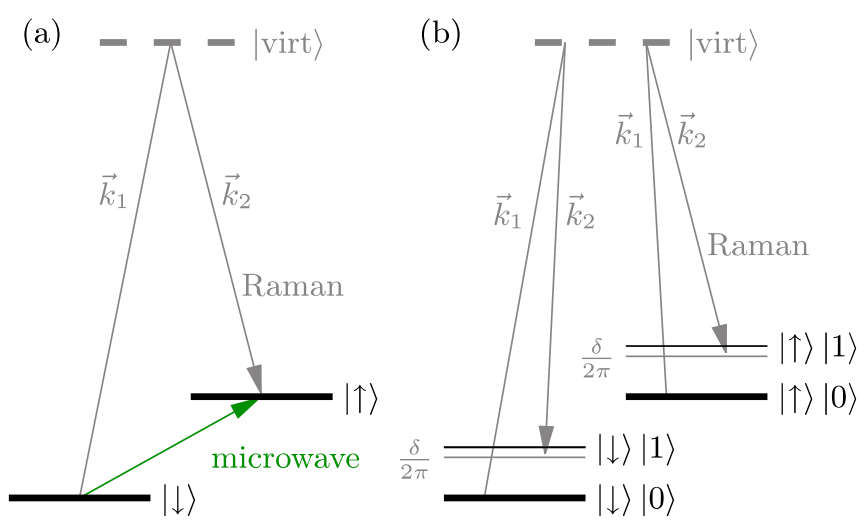

Figure 5. Implementations of different interaction types for hyperfine/Zeeman qubits. (a) An operation of type (a) can be implemented, for example, by two-photon stimulated-Raman transitions driven by a pair of laser beams (shown without motional dependence) or directly by a microwave field. These types of interactions can be used for single-qubit gates in QC and to simulate the effective magnetic field in the simulation of quantum spin Hamiltonians. (b) State-dependent forces (see type (c) in the text) can be created by two beams detuned by approximately the frequency of a motional mode. This interaction is used in the geometric phase gate for the displacement pulse [110,123] or in the simulation of the quantum Ising Hamiltonian to create the effective spin-spin interaction $[21,47]$.

Coupling via optical fields. Optical qubit states can be linked by a single, nearly resonant laser beam with frequency $\omega_{\mathrm{I}}$ and wave vector $\vec{k}_{\mathrm{I}}$. Due to the lifetime of the $|\uparrow\rangle$ state of $1 / \Gamma \sim 1 \mathrm{~s}$, the linewidth of the laser has to be very narrow $(\sim 1 \mathrm{~Hz})$. Operations (a) and (b) can be implemented directly (see section 3.2). State-dependent forces (c) can be provided by a bichromatic light field (see, for example, [85-87] and also section 3.3).

In hyperfine/Zeeman qubits, the single laser beam can be substituted by two beams with frequencies $\omega_{1}, \omega_{2}$ and wavevectors $\vec{k}_{1}, \vec{k}_{2}$ driving two-photon stimulated-Raman transitions. The beams are detuned by $\Delta \gg \Gamma$ from a third level, for example, a P level (compare figure 4) with a typical lifetime $1 / \Gamma$ of the order of few nanoseconds. In the mathematical treatment, this third level can be adiabatically eliminated for large detunings and the interaction gains the form of an interaction with a single beam of frequency $\omega_{\mathrm{I}}=$ $\left|\omega_{1}-\omega_{2}\right|$ and wavevector $\vec{k}_{\mathrm{I}}=\vec{k}_{1}-\vec{k}_{2}$. The requirement of a narrow linewidth holds only for the difference frequency $\omega_{\mathrm{I}}$, which can be fulfilled comparatively easily: the two beams can be generated from the same laser using acousto-optical modulators driven by a stable microwave source, while the requirements on the frequency stability of the laser are relaxed. For operation (a), the frequency $\omega_{\mathrm{I}}$ has to (approximately) meet the transition frequency of the qubit states (see figure $5(a)$ ). For operation (b), $\vec{k}_{\mathrm{I}}$ in addition must not vanish to achieve a sufficient momentum transfer to the ions, see figure 4 and section 3.2. Therefore, the two beams are typically orthogonal $\left(\left|\vec{k}_{\mathrm{I}}\right| \approx \sqrt{2}\left|\vec{k}_{1}\right|\right)$ or counter-propagating $\left(\left|\vec{k}_{\mathrm{I}}\right| \approx 2\left|\vec{k}_{1}\right|\right)$. The state-dependent forces (c) can be implemented by nearly resonant beams $\left(\omega_{\mathrm{I}} \approx 0\right)$ and beam geometries as for operation (b) (see figure 5(b), sections 3.3-3.5 and section 4).

The main technical drawback of using two-photon stimulated-Raman transitions is decoherence due to spontaneous emission after off-resonantly populating the third level. This limitation can be mitigated by increasing the detuning $\Delta$ and the intensities $I_{1 / 2}$ of the beams, since the interaction strength scales with $I_{1 / 2} / \Delta$, while the spontaneous emission rate scales with $I_{1 / 2} / \Delta^{2}$.

Coupling via microwave fields. Alternatively, transitions between the electronic states in hyperfine/Zeeman qubits can be driven laser-less by microwave fields. This allows the direct realization of operation (a). However, due to the comparatively long wavelength and the related small momentum transfer $\left(\left|\hbar \vec{k}_{\mathrm{I}}\right| \rightarrow 0\right)$, only negligible coupling to the motional modes can be achieved directly and additional efforts are required to provide operations (b) and (c) [88,89]: by applying a static magnetic field gradient along the axis of an ion chain, the transition frequency between $|\downarrow\rangle$ and $|\uparrow\rangle$ becomes sitedependent due to position-dependent Zeeman shifts. The ions can be individually addressed by applying microwave fields with these site-dependent frequencies $\omega_{\text {I }}$. In addition, this causes state-dependent forces as in the Stern-Gerlach experiment and allows for coupling to the motional modes. The main challenge here is to provide sufficiently large magnetic field gradients and to cope with state-dependent transition frequencies, if high operational fidelities are required (see, for example, [90]).

As an alternative to the static magnetic field gradients, alternating magnetic fields due to microwave currents in electrodes of surface-electrode traps (see section 6) have been proposed [91] and first promising results have been achieved $[92,93]$. Due to the small height of the ion above the electrode in this type of trap, a sufficiently large ac Zeeman shift can be generated, which can be treated analogously to the ac Stark shift created by laser beams in two-photon stimulated-Raman transitions discussed above. However, the small height above the electrodes leads to further challenges (compare section 6) and high microwave powers are required.

\subsection{Initialization and readout}

Initialization of motional and electronic states. The initialization into one of the qubit states, for example $|\downarrow\rangle$, can be achieved with near-unity efficiency by optical pumping [94]. Regarding the motional modes, the initialization includes Doppler cooling in all three dimensions leading to a thermal state with an average phonon number $\bar{n}$ of typically a few to ten quanta. This pre-cooling is required to reach the Lamb-Dicke regime (see section 3.2), where subsequent resolved sideband cooling [95-97] or cooling utilizing electromagnetically induced transparency can be applied $[98,99]$. These cooling schemes lead close to the motional ground state $|0\rangle$ ( $n=0$ with probability of $98 \%$ in [97]) of the dedicated modes.

Readout of electronic and motional states. We distinguish the two electronic states by observing state-dependent laser fluorescence. The dipole allowed transition to an excited state starting in the state $|\downarrow\rangle$ is driven resonantly (see the transition labelled 'BD' in figure 4) in a closed cycle completed by spontaneous emission back to the state $|\downarrow\rangle$ due to selection rules. For state $|\uparrow\rangle$ the detection laser is off-resonant. The ion 
therefore appears 'bright' for $|\downarrow\rangle$, while it remains 'dark' for $|\uparrow\rangle$ [100-103]. Typically, a few per mill of the scattered photons are detected by a photomultiplier tube or a CCD camera. The fidelity of this detection scheme has been shown experimentally to exceed $99.99 \%$ for averaged and even individual measurements [104, 105]. However, additional possibilities to enhance the detection efficiency, for example by methods developed for QC using ancilla qubits [106-108], cannot be applied to analogue QS, since all ions participate during the simulation. For the detection of the motional state, it can be mapped to the electronic state via an operation of type (b) and derived from the result of the spin state detection described above [109].

\section{Theoretical excursion}

The following calculations (section 3.1 and 3.2) are the mathematical description of the toolbox that is available for both QCs and QSs. Detailed discussions can be found, for example, in [50] or [51]. We summarize important equations in the following and extend the mathematical description to be applicable to scaled approaches of QSs, for example, two-dimensional arrays of ions in individual traps. We will continue with the description of an implementation of the effective spin-spin interaction for ions appearing in quantum spin Hamiltonians. In order to investigate it isolated from other interactions, we will first discuss it from the point of view of quantum gates [110] in sections 3.3 and 3.4. Finally, in section 3.5, we use all tools to derive and discuss the quantum Ising Hamiltonian based on [21] as an example. The mathematical descriptions will be required to pursue proposals described in section 5.2.

This section aims at deriving the mathematical treatment of the simulation of a quantum Ising Hamiltonian. It underlines the approximations and transformations applied in these calculations and discusses the related corrections compared with the ideal quantum Ising model, which grow in importance in regard to scaled systems described in section 6. Readers who are more interested in a pictorial description of the simulation of a quantum Ising Hamiltonian may skip this section and continue to section 4 .

\subsection{Theoretical basics}

In the following we consider two-level systems only. The Hamiltonian describing the energy of the electronic states of $N$ such systems is given by

$$
\hat{\mathcal{H}}_{\mathrm{e}}=\sum_{i=1}^{N} \frac{\hbar \omega_{\uparrow \downarrow}}{2} \hat{\sigma}_{z}^{(i)}+\underbrace{N \hbar \frac{\omega_{\uparrow}+\omega_{\downarrow}}{2}}_{=\text {const (omitted) }},
$$

where $\hbar \omega_{\uparrow / \downarrow}$ denote the energies of the states $|\downarrow\rangle$ and $|\uparrow\rangle$, respectively, $\omega_{\uparrow \downarrow}:=\omega_{\uparrow}-\omega_{\downarrow}$, and the operator $\hat{\sigma}_{z}^{(i)}$ the Pauli operator (compare appendix B, equation (B.1)) acting on the $i$ th ion.

The ions are considered to be trapped in a common harmonic potential or several individual potentials, which can be approximated to harmonic order. The corresponding Hamiltonian in terms of the normal modes of the oscillation reads

$$
\hat{\mathcal{H}}_{\mathrm{m}}=\sum_{m=1}^{3 N} \hbar \omega_{m}\left(\hat{a}_{m}^{\dagger} \hat{a}_{m}+\frac{1}{2}\right) .
$$

Here, $\hat{a}_{m}$ and $\hat{a}_{m}^{\dagger}$ are the annihilation and creation operators of the $m$ th mode, respectively, and $\omega_{m}$ the corresponding frequency. In the following, the constant terms $\hbar \omega_{m} / 2$ will also be omitted and the abbreviation $\hat{\mathcal{H}}_{0}:=\hat{\mathcal{H}}_{\mathrm{e}}+\hat{\mathcal{H}}_{\mathrm{m}}$ will be used.

An interaction of an ion with the electric field $\vec{E}$ of a laser beam is described by $-\hat{\vec{\mu}} \cdot \vec{E}(\vec{r}, t)$, where $\hat{\vec{\mu}}$ denotes the electric dipole operator for the transition $|\downarrow\rangle \leftrightarrow|\uparrow\rangle$ and $\vec{E}(\vec{r}, t)$ the field at the site of the ion. The Hamiltonian describing the interaction of the field with $N$ ions becomes

$$
\hat{\mathcal{H}}_{\mathrm{I}}=\sum_{i=1}^{N} \hbar \Omega_{\mathrm{I}}^{(i)}\left(\mathrm{e}^{\mathrm{i}\left(\vec{k}_{\mathrm{I}}^{(i)} \cdot \hat{\hat{r}}^{(i)}-\omega_{\mathrm{I}} t+\varphi_{\mathrm{I}}^{(i)}\right)}+\text { h.c. }\right) \hat{\kappa}^{(i)} .
$$

Here, $\Omega_{\mathrm{I}}^{(i)}=-\mu E^{(i)} / 2 \in \mathbb{R}$ is the interaction strength at site $i, \vec{k}_{\mathrm{I}}^{(i)}$ the wavevector at site $i, \hat{\vec{r}}^{(i)}$ the position of the $i$ th ion, $\omega_{\mathrm{I}}$ the frequency of the field and $\varphi_{\mathrm{I}}^{(i)}$ an additional phase. In the most general form, the operator $\hat{\kappa}^{(i)}$ can be expressed as a linear combination of Pauli operators $\hat{\sigma}_{x / y / z}^{(i)}$ and the identity operator $\hat{\mathbb{1}}^{(i)}$ (see appendix B):

$$
\hat{\kappa}^{(i)}:=\alpha_{0} \hat{\mathbb{1}}^{(i)}+\alpha_{1} \hat{\sigma}_{x}^{(i)}+\alpha_{2} \hat{\sigma}_{y}^{(i)}+\alpha_{3} \hat{\sigma}_{z}^{(i)},
$$

with the prefactors $\alpha_{j} \in \mathbb{R}$, which are determined by the polarization of the electric field and angular momenta of the states encoding $|\downarrow\rangle$ and $|\uparrow\rangle$ (see also section 3.4 for some examples).

The position operator $\hat{\vec{r}}^{(i)}$ in equation (3.3) is decomposed into the equilibrium position $\vec{x}_{0}^{(i)}$ and the displacement $\hat{\vec{x}}^{(i)}=$ $\hat{\vec{r}}^{(i)}-\vec{x}_{0}^{(i)}$. The terms $\vec{k}_{\mathrm{I}}^{(i)} \cdot \vec{x}_{0}^{(i)}$ give rise to a constant phase, which we absorb into $\varphi_{\mathrm{I}}^{(i)}+\vec{k}_{\mathrm{I}}^{(i)} \cdot \vec{x}_{0}^{(i)} \rightarrow \varphi_{\mathrm{I}}^{(i)}$.

The displacement of the ion from its equilibrium position $\hat{\vec{x}}^{(i)}$ is expressed in terms of the normal modes of motion

$$
\hat{\vec{x}}^{(i)}=\sum_{m=1}^{3 N}\left(b_{m, i} \hat{q}_{m} \vec{e}_{X}+b_{m, i+N} \hat{q}_{m} \vec{e}_{Y}+b_{m, i+2 N} \hat{q}_{m} \vec{e}_{Z}\right)
$$

where $b_{m, i}$ are the elements of an (orthogonal) transformation matrix (compare appendix A, equation (A.8)). Expressing the operators $\hat{q}_{m}$ of the normal modes in terms of the creation and annihilation operators yields

$$
\hat{q}_{m}=q_{m 0}\left(\hat{a}_{m}+\hat{a}_{m}^{\dagger}\right) \quad \text { with } \quad q_{m 0}:=\sqrt{\frac{\hbar}{2 M \omega_{m}}},
$$

where $M$ denotes the mass of one ion. Hence, the scalar product appearing in the Hamiltonian yields

$$
\vec{k}_{\mathrm{I}}^{(i)} \cdot \hat{\vec{x}}^{(i)}=\sum_{m=1}^{3 N} \eta_{m}^{(i)}\left(\hat{a}_{m}+\hat{a}_{m}^{\dagger}\right),
$$

where the Lamb-Dicke parameter of the $m$ th mode and $i$ th site has been introduced:

$\eta_{m}^{(i)}:=q_{m 0}\left(b_{m, i} \vec{k}_{\mathrm{I}}^{(i)} \cdot \vec{e}_{X}+b_{m, i+N} \vec{k}_{\mathrm{I}}^{(i)} \cdot \vec{e}_{Y}+b_{m, i+2 N} \vec{k}_{\mathrm{I}}^{(i)} \cdot \vec{e}_{Z}\right)$. 
To summarize, the interaction term of the Hamiltonian gains the form

$$
\hat{\mathcal{H}}_{\mathrm{I}}=\sum_{i=1}^{N} \hbar \Omega_{\mathrm{I}}^{(i)}\left(\mathrm{e}^{\mathrm{i}\left[\sum_{m=1}^{3 N} \eta_{m}^{(i)}\left(\hat{a}_{m}+\hat{a}_{m}^{\dagger}\right)-\omega_{\mathrm{I}} t+\varphi_{\mathrm{I}}^{(i)}\right]}+\text { h.c. }\right) \hat{\kappa}^{(i)} \text {. }
$$

The transformation into the interaction picture

$$
\hat{\mathcal{H}}_{\mathrm{I}}^{\prime}=\hat{U}_{0}^{\dagger} \hat{\mathcal{H}}_{\mathrm{I}} \hat{U}_{0} \quad \text { with } \quad \hat{U}_{0}:=\mathrm{e}^{-\mathrm{i} \hat{\mathcal{H}}_{0} t / \hbar}
$$

can be carried out for each site separately, hence

$$
\hat{\mathcal{H}}_{\mathrm{I}}^{(i)}=\hat{U}_{0}^{(i)^{\dagger}} \hat{\mathcal{H}}_{\mathrm{I}}^{(i)} \hat{U}_{0}^{(i)} \quad \text { with } \quad \hat{U}_{0}^{(i)}:=\mathrm{e}^{-\mathrm{i} \hat{\mathcal{H}}_{0}^{(i)} t / \hbar},
$$

where $\hat{\mathcal{H}}_{\mathrm{I}}^{(i)}$ is the Hamiltonian corresponding to the $i$ th ion and $\hat{\mathcal{H}}_{\mathrm{I}}=\sum_{i=1}^{N} \hat{\mathcal{H}}_{\mathrm{I}}^{(i)}$.

The operator $\hat{\kappa}^{(i)}$ (equation (3.4)) related to the electronic states reads as follows in the interaction picture (compare equation (B.15)):

$$
\begin{aligned}
\hat{\kappa}^{\prime(i)} & =\mathrm{e}^{\mathrm{i} \omega_{\uparrow \downarrow} t \hat{\sigma}_{z}^{(i)} / 2} \hat{\kappa}^{(i)} \mathrm{e}^{-\mathrm{i} \omega_{\uparrow \downarrow} t \hat{\sigma}_{z}^{(i)} / 2} \\
& =\frac{1}{2}\left[\alpha_{0} \hat{\mathbb{1}}^{(i)}+\left(\alpha_{1}+\frac{\alpha_{2}}{\mathrm{i}}\right) \mathrm{e}^{\mathrm{i} \omega_{\uparrow \downarrow} t} \hat{\sigma}_{+}^{(i)}+\alpha_{3} \hat{\sigma}_{z}^{(i)}\right]+\text { h.c. }
\end{aligned}
$$

Here, we introduced $\hat{\sigma}_{+}^{(i)}:=\hat{\sigma}_{x}^{(i)}+\mathrm{i} \hat{\sigma}_{y}^{(i)}$ and $\hat{\sigma}_{-}^{(i)}:=\hat{\sigma}_{x}^{(i)}-\mathrm{i} \hat{\sigma}_{y}^{(i)}$.

The terms in equation (3.9) containing the motional operators transform as follows (compare equation (C.10)):

$$
\begin{aligned}
& \mathrm{e}^{\mathrm{i} \omega_{m} t \hat{a}_{m}^{\dagger} \hat{a}_{m}} \mathrm{e}^{\mathrm{i} \eta_{m}^{(i)}\left(\hat{a}_{m}+\hat{a}_{m}^{\dagger}\right)} \mathrm{e}^{-\mathrm{i} \omega_{m} t \hat{a}_{m}^{\dagger} \hat{a}_{m}} \\
& =\exp \left(\mathrm{i} \eta_{m}^{(i)}\left[\hat{a}_{m} \mathrm{e}^{-\mathrm{i} \omega_{m} t}+\hat{a}_{m}^{\dagger} \mathrm{e}^{\mathrm{i} \omega_{m} t}\right]\right) .
\end{aligned}
$$

Hence, it yields the following expression for the complete Hamiltonian in the interaction picture:

$$
\begin{aligned}
\hat{\mathcal{H}}_{\mathrm{I}}^{\prime(i)}= & \hbar \Omega_{\mathrm{I}}^{(i)}\left\{\operatorname { e x p } \left(\mathrm { i } \left[\sum_{m=1}^{3 N} \eta_{m}^{(i)}\left(\hat{a}_{m} \mathrm{e}^{-\mathrm{i} \omega_{m} t}+\hat{a}_{m}^{\dagger} \mathrm{e}^{\mathrm{i} \omega_{m} t}\right)\right.\right.\right. \\
& \left.\left.\left.-\omega_{\mathrm{I}} t+\varphi_{\mathrm{I}}^{(i)}\right]\right)+ \text { h.c. }\right\} \hat{\kappa}^{\prime(i)} .
\end{aligned}
$$

At this point fast rotating terms which average out on short timescales are neglected (rotating wave approximation, RWA). For $\Omega_{\mathrm{I}}^{(i)} \ll \omega_{\uparrow \downarrow}$ we distinguish between two cases: In the first case, $\omega_{\mathrm{I}} \ll \omega_{\uparrow \downarrow}$, terms containing $\mathrm{e}^{ \pm \mathrm{i} \omega_{\uparrow \downarrow} t}$ are neglected (see figure 5(b) for an example of an implementation). (If $\alpha_{1}=\alpha_{2}=0$, nothing will change and the Hamiltonian will still be exact.) In the second case, $\left|\omega_{\mathrm{I}}-\omega_{\uparrow \downarrow}\right| \ll \omega_{\uparrow \downarrow}$, all terms but $\mathrm{e}^{ \pm \mathrm{i}\left(\omega_{\uparrow \downarrow}-\omega_{\mathrm{I}}\right) t}$ are neglected (see figure 5(a)):

$$
\begin{gathered}
\hat{\mathcal{H}}_{\mathrm{I}}^{(i)(\mathrm{RWA})}=\hbar \Omega_{\mathrm{I}}^{(i)} \exp \left(\mathrm { i } \left[\sum_{m=1}^{3 N} \eta_{m}^{(i)}\left(\hat{a}_{m} \mathrm{e}^{-\mathrm{i} \omega_{m} t}+\hat{a}_{m}^{\dagger} \mathrm{e}^{\mathrm{i} \omega_{m} t}\right)\right.\right. \\
\left.\left.-\omega_{\mathrm{I}} t+\varphi_{\mathrm{I}}^{(i)}\right]\right)\left(\alpha_{0} \hat{\mathbb{1}}^{(i)}+\alpha_{3} \hat{\sigma}_{z}^{(i)}\right)+\text { h.c. }
\end{gathered}
$$$$
\text { for } \omega_{\mathrm{I}} \ll \omega_{\uparrow \downarrow} \text {, }
$$

$$
\begin{aligned}
& \hat{\mathcal{H}}_{\mathrm{I}}^{(i)(\mathrm{RWA})}=\frac{\hbar}{2} \Omega_{\mathrm{I}}^{(i)} \exp \left(\mathrm { i } \left[\sum_{m=1}^{3 N} \eta_{m}^{(i)}\left(\hat{a}_{m} \mathrm{e}^{-\mathrm{i} \omega_{m} t}+\hat{a}_{m}^{\dagger} \mathrm{e}^{\mathrm{i} \omega_{m} t}\right)\right.\right. \\
& \left.\left.\quad-\left(\omega_{\mathrm{I}}-\omega_{\uparrow \downarrow}\right) t+\varphi_{\mathrm{I}}^{(i)}\right]\right)\left(\alpha_{1}+\frac{\alpha_{2}}{\mathrm{i}}\right) \hat{\sigma}_{+}^{(i)}+\text { h.c. } \\
& \quad \text { for }\left|\omega_{\mathrm{I}}-\omega_{\uparrow \downarrow}\right| \ll \omega_{\uparrow \downarrow} .
\end{aligned}
$$

\section{2. $\hat{\sigma}_{x} / \hat{\sigma}_{y}$ interaction}

The time evolution corresponding to $\hat{\mathcal{H}}_{\mathrm{I}}^{\prime(i)(\mathrm{RWA})}$ in equation (3.17) is involved. The time evolution is calculated for a single ion $i$ and a single motional mode $m$, for example, in [50,51]. As some simplifications (Lamb-Dicke regime, see below) are not always justified for experiments, we will summarize this calculation here. (The index $m$ is skipped in this section.)

In this case the Hamiltonian simplifies to

$$
\begin{aligned}
& \hat{\mathcal{H}}_{\mathrm{I}}^{\prime(\mathrm{RWA})}=\frac{\hbar}{2} \Omega_{\mathrm{I}} \exp \left(\mathrm{i}\left[\eta\left(\hat{a} \mathrm{e}^{-\mathrm{i} \omega t}+\hat{a}^{\dagger} \mathrm{e}^{\mathrm{i} \omega t}\right)-\left(\omega_{\mathrm{I}}-\omega_{\uparrow \downarrow}\right) t+\varphi_{\mathrm{I}}\right]\right) \\
& \quad \times\left(\alpha_{1}+\frac{\alpha_{2}}{\mathrm{i}}\right) \hat{\sigma}_{+}+\text {h.c. }
\end{aligned}
$$

Writing the state vector in the basis of electronic states $|s\rangle$ and motional Fock states $|n\rangle$,

$$
|\psi(t)\rangle=\sum_{s \in\{\downarrow, \uparrow\}} \sum_{n} c_{s, n}(t)|s, n\rangle,
$$

the Schrödinger equation yields

$$
\mathrm{i} \hbar \dot{c}_{s^{\prime}, n^{\prime}}(t)=\sum_{s \in\{\downarrow, \uparrow\}} \sum_{n}\left\langle s^{\prime}, n^{\prime}\left|\hat{\mathcal{H}}_{\mathrm{I}}^{\prime(\mathrm{RWA})}\right| s, n\right\rangle c_{s, n}(t) .
$$

Matrix elements of the Hamiltonian vanish for $s^{\prime}=s$. We obtain for the non-vanishing matrix elements $[95,111]$ (compare appendix D)

$$
\begin{aligned}
\left\langle\uparrow, n^{\prime}\left|\hat{\mathcal{H}}_{\mathrm{I}}^{\prime(\mathrm{RWA})}\right| \downarrow, n\right\rangle=\frac{\hbar}{2} \Omega_{\mathrm{I}} \mathrm{e}^{\mathrm{i}\left(-\left(\omega_{\mathrm{I}}-\omega_{\uparrow \downarrow}\right) t+\varphi_{\mathrm{I}}\right)}\left(\alpha_{1}+\frac{\alpha_{2}}{\mathrm{i}}\right) \\
\times\left\langle n^{\prime}\left|\hat{D}\left(\mathrm{i} \eta \mathrm{e}^{\mathrm{i} \omega t}\right)\right| n\right\rangle\left\langle\uparrow\left|\hat{\sigma}_{+}\right| \downarrow\right\rangle \\
=\hbar \Omega_{n^{\prime}, n}\left(\alpha_{1}+\frac{\alpha_{2}}{\mathrm{i}}\right) \mathrm{i}^{\left|n^{\prime}-n\right|} \mathrm{e}^{\mathrm{i}\left(\left[\left(n^{\prime}-n\right) \omega-\left(\omega_{\mathrm{I}}-\omega_{\uparrow \downarrow}\right)\right] t+\varphi_{\mathrm{I}}\right)},
\end{aligned}
$$

where $\hat{D}(\lambda):=\mathrm{e}^{\lambda \hat{a}^{\dagger}-\lambda^{*} \hat{a}}$ denotes the displacement operator and

$$
\Omega_{n^{\prime}, n}:=\Omega_{\mathrm{I}} \mathrm{e}^{-\eta^{2} / 2} \eta^{\left|n^{\prime}-n\right|} \sqrt{\frac{n_{<} !}{n_{>} !}} L_{n_{<}}^{\left(\left|n^{\prime}-n\right|\right)}\left(\eta^{2}\right) .
$$

Here, $L_{n}^{(\alpha)}(x)$ are the associated Laguerre polynomials, $n_{<}:=$ $\min \left(n^{\prime}, n\right)$, and $n_{>}:=\max \left(n^{\prime}, n\right)$. Analogously, we obtain $\left\langle\downarrow, n\left|\hat{\mathcal{H}}_{\mathrm{I}}^{\prime(\mathrm{RWA})}\right| \uparrow, n^{\prime}\right\rangle=\left\langle\uparrow, n^{\prime}\left|\hat{\mathcal{H}}_{\mathrm{I}}^{\prime(\mathrm{RWA})}\right| \downarrow, n\right\rangle^{*}$

We define $\delta:=\left(\omega_{\mathrm{I}}-\omega_{\uparrow \downarrow}\right)-\left(n^{\prime}-n\right) \omega$. For small detunings $|\delta| \ll \omega$ and interaction strengths $\left|\Omega_{n^{\prime}, n}\right| \ll \omega$ (resolved sideband regime), we apply an RWA neglecting terms rotating faster than $\mathrm{e}^{ \pm \mathrm{i} \delta t}$. Equation (3.20) can then be solved for each subset $\left|n^{\prime}, \uparrow\right\rangle$ and $|n, \downarrow\rangle$ separately:

$\dot{c}_{\uparrow, n^{\prime}}(t)=-\mathrm{i} \Omega_{n^{\prime}, n}\left(\alpha_{1}+\frac{\alpha_{2}}{\mathrm{i}}\right) \mathrm{i}^{\left|n^{\prime}-n\right|} \mathrm{e}^{-\mathrm{i}\left(\delta t-\varphi_{\mathrm{I}}\right)} c_{\downarrow, n}(t)$,

$\dot{c}_{\downarrow, n}(t)=-\mathrm{i} \Omega_{n^{\prime}, n}\left(\alpha_{1}+\frac{\alpha_{2}}{\mathrm{i}}\right)^{*}(-\mathrm{i})^{\left|n^{\prime}-n\right|} \mathrm{e}^{\mathrm{i}\left(\delta t+\varphi_{\mathrm{I}}\right)} c_{\uparrow, n^{\prime}}(t)$.

The solution of the system of differential equations yields Rabi oscillations between the states $|\downarrow, n\rangle \leftrightarrow\left|\uparrow, n^{\prime}\right\rangle$ (compare appendix E): 


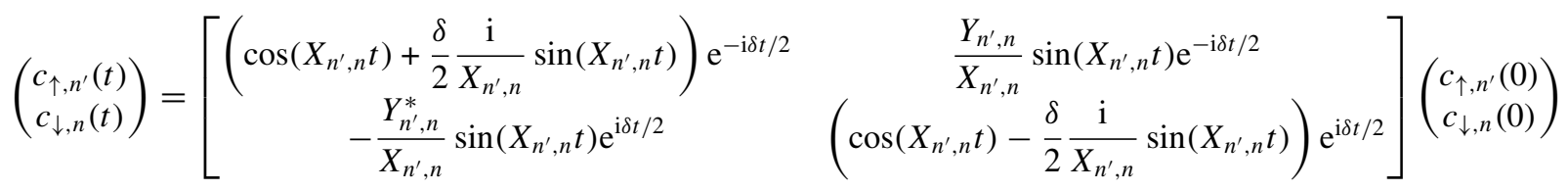

with $Y_{n^{\prime}, n}:=-\mathrm{i} \Omega_{n^{\prime}, n}\left(\alpha_{1}+\alpha_{2} / \mathrm{i}\right) \mathrm{i}^{\left|n^{\prime}-n\right|} \mathrm{e}^{\mathrm{i} \varphi_{\mathrm{I}}}$ and $X_{n^{\prime}, n}:=$ $\sqrt{\left(\delta^{2} / 4\right)+\left|Y_{n^{\prime}, n}\right|^{2}}$.

In the Lamb-Dicke regime, $\eta\left\langle\left(\hat{a}+\hat{a}^{\dagger}\right)^{2}\right\rangle^{1 / 2} \ll 1$, equation (3.22) can be expanded to first order in $\eta$ :

$\Omega_{n-1, n}^{(\mathrm{LDR})}=\Omega_{\mathrm{I}} \eta \sqrt{n} \quad$ (first red sideband),

$\Omega_{n, n}^{(\mathrm{LDR})}=\Omega_{\mathrm{I}} \quad$ (carrier),

$\Omega_{n+1, n}^{(\mathrm{LDR})}=\Omega_{\mathrm{I}} \eta \sqrt{n+1} \quad$ (first blue sideband).

Successive red sideband transitions $|\downarrow\rangle|n\rangle \rightarrow|\uparrow\rangle|n-1\rangle$ followed by dissipative repumping to $|\downarrow\rangle|n-1\rangle$ with high probability are routinely used for sideband cooling close to the motional ground state $|n=0\rangle$ [95-97].

If the Lamb-Dicke parameter becomes effectively zero, the motional dependence will vanish (see equations (3.26) and (3.28)). The only remaining transition is the carrier transition equation (3.27) affecting the electronic states only. This is the case, for example, for two-photon stimulated-Raman transitions with co-propagating beams or for microwave driven transitions in hyperfine qubits, where $\vec{k}_{\mathrm{I}} \approx 0$. In systems with more than one ion, the ions will not be motionally coupled. That is why equation (3.25) also holds for each site separately in such systems. order in the Lamb-Dicke parameters $\eta_{m}^{(i)}$. A subsequent RWA neglecting terms rotating faster than $\mathrm{e}^{ \pm \mathrm{i} \delta_{m} t}$ with $\delta_{m}:=\omega_{\mathrm{I}}-\omega_{m}$ yields

$$
\begin{aligned}
& \hat{\mathcal{H}}_{\mathrm{I}}^{(i)(\mathrm{LDR})}=\hbar \Omega_{\mathrm{I}}^{(i)} \mathrm{e}^{\mathrm{i}\left(-\omega_{\mathrm{I}} t+\varphi_{\mathrm{I}}^{(i)}\right)} \\
& \times\left[1+\mathrm{i} \sum_{m=1}^{3 N} \eta_{m}^{(i)}\left(\hat{a}_{m} \mathrm{e}^{-\mathrm{i} \omega_{m} t}+\hat{a}_{m}^{\dagger} \mathrm{e}^{\mathrm{i} \omega_{m} t}\right)\right] \\
& \times\left(\alpha_{0} \hat{\mathbb{1}}^{(i)}+\alpha_{3} \hat{\sigma}_{z}^{(i)}\right)+\text { h.c. } \\
& \Rightarrow \hat{\mathcal{H}}_{\mathrm{I}}^{(i)(\mathrm{LDR})(\mathrm{RWA})}=\mathrm{i} \hbar \Omega_{\mathrm{I}}^{(i)} \sum_{m=1}^{3 N} \eta_{m}^{(i)} \mathrm{e}^{\mathrm{i}\left(-\delta_{m} t+\varphi_{\mathrm{I}}^{(i)}\right)} \hat{a}_{m}^{\dagger} \\
& \quad \times\left(\alpha_{0} \hat{\mathbb{1}}^{(i)}+\alpha_{3} \hat{\sigma}_{z}^{(i)}\right)+\text { h.c. }
\end{aligned}
$$

Note that equation (3.32) breaks up into a sum over terms that depend on only one mode $m$ and one site $i$ each.

With the excursion in appendix $F$ the total time evolution operator in the interaction picture reads

$$
\begin{aligned}
\hat{U}_{\mathrm{I}}^{\prime(\mathrm{LDR})(\mathrm{RWA})}\left(t, t_{0}\right)= & \exp \left(\mathrm{i}\left[\sum_{i=1}^{N} \sum_{m=1}^{3 N} \frac{\Omega_{\mathrm{I}}^{(i)} \eta_{m}^{(i)}}{\delta_{m}}\left(\mathrm{e}^{-\mathrm{i} \delta_{m}\left(t-t_{0}\right)}-1\right) \mathrm{e}^{-\mathrm{i} \delta_{m} t_{0}} \mathrm{e}^{\mathrm{i} \varphi_{\mathrm{I}}^{(i)}} \hat{a}_{m}^{\dagger}\left(\alpha_{0} \hat{\mathbb{1}}^{(i)}+\alpha_{3} \hat{\sigma}_{z}^{(i)}\right)+\text { h.c. }\right]\right) \\
& \times \exp \left(-\mathrm{i} \sum_{i=1}^{N} \sum_{j=1}^{N} \sum_{m=1}^{3 N} \frac{\Omega_{\mathrm{I}}^{(i)} \Omega_{\mathrm{I}}^{(j)} \eta_{m}^{(i)} \eta_{m}^{(j)}}{\delta_{m}^{2}}\left(\alpha_{0} \hat{\mathbb{1}}^{(i)}+\alpha_{3} \hat{\sigma}_{z}^{(i)}\right) \otimes\left(\alpha_{0} \hat{\mathbb{1}}^{(j)}+\alpha_{3} \hat{\sigma}_{z}^{(j)}\right)\right. \\
& \left.\times\left[\delta_{m}\left(t-t_{0}\right) \cos \left(\varphi_{\mathrm{I}}^{(i)}-\varphi_{\mathrm{I}}^{(j)}\right)-\sin \left(\delta_{m}\left(t-t_{0}\right)-\left(\varphi_{\mathrm{I}}^{(i)}-\varphi_{\mathrm{I}}^{(j)}\right)\right)\right]\right) .
\end{aligned}
$$

Equation (3.25) simplifies for resonant carrier transitions $(\delta=0)$ and a pure $\hat{\sigma}_{x}$ interaction $\left(\alpha_{1}=1\right.$ and $\left.\alpha_{2}=0\right)$ :

$$
\left(\begin{array}{l}
c_{\uparrow, n}(t) \\
c_{\downarrow, n}(t)
\end{array}\right)=\hat{R}(\vartheta, \varphi)\left(\begin{array}{l}
c_{\uparrow, n}(0) \\
c_{\downarrow, n}(0)
\end{array}\right),
$$

where

$$
\hat{R}(\vartheta, \varphi):=\left(\begin{array}{cc}
\cos (\vartheta / 2) & -\mathrm{ie}^{\mathrm{i} \varphi} \sin (\vartheta / 2) \\
-\mathrm{ie}^{-\mathrm{i} \varphi} \sin (\vartheta / 2) & \cos (\vartheta / 2)
\end{array}\right),
$$

$\vartheta:=2 \Omega_{n, n} t$ and $\varphi:=\varphi_{\mathrm{I}}$. The rotation matrix $\hat{R}(\pi / 2, \varphi)$ describes a $\pi / 2$-pulse and $\hat{R}(\pi, \varphi)$ a $\pi$-pulse with phase $\varphi$.

\subsection{Effective $\hat{\sigma}_{z} \otimes \hat{\sigma}_{z}$ interaction}

We will now discuss the case of equation (3.16) with $\alpha_{1}=$ $\alpha_{2}=0$. Hence, we omit the superscript of the Hamiltonian indicating an RWA. In the Lamb-Dicke regime, $\eta_{m}^{(i)}\left\langle\left(\hat{a}_{m}+\right.\right.$ $\left.\left.\hat{a}_{m}^{\dagger}\right)^{2}\right\rangle^{1 / 2} \ll 1$, the Hamiltonian can be expanded to first
The interaction described by equation (3.33) can be interpreted as follows: the first exponential function has the form of a displacement operator $\hat{D}(\lambda)=\mathrm{e}^{\lambda \hat{a}^{\dagger}-\lambda^{*} \hat{a}}$, which leads to a displacement of a coherent state by $\lambda$ in phase space. Due to the $\left(\mathrm{e}^{-\mathrm{i} \delta_{m}\left(t-t_{0}\right)}-1\right)$ proportionality of the exponent, the trajectory for a coherent state of each mode describes a circle in phase space (or a straight line in the limit $\delta_{m}=0$ ). The coherent state returns to its initial position at times $T_{m}=2 \pi l / \delta_{m}$ with $l \in \mathbb{N}$, where the exponent vanishes. The second exponential can be expanded into a $\hat{\sigma}_{z} \otimes \hat{\sigma}_{z}$ interaction, a $\hat{\sigma}_{z}$ interaction and a global phase. The $\hat{\sigma}_{z} \otimes \hat{\sigma}_{z}$ terms give rise to a geometric phase, which increases in time $t$, and the $\hat{\sigma}_{z}$ terms lead to a dynamic phase $[112,113]$. The area in phase space enclosed by the trajectory is proportional to these phases.

\subsection{Geometric phase gates}

The collective interaction of multiple ions with the same laser(s) has been proposed for the implementation of quantum 
gates [85, 114-117]. These gates are described in the $z$ basis by equation (3.33) and have been first implemented in $[110,118]$. Mølmer-Sørensen gates can be mathematically treated analogously in a rotated basis and are described in detail in [86, 87]. Implementations are reported in [119-122].

We will exemplarily discuss geometric phase gates based on the $\hat{\sigma}_{z} \otimes \hat{\sigma}_{z}$ terms in equation (3.33) in the following. They offer excellent tools to investigate a pure $\hat{\sigma}_{z} \otimes \hat{\sigma}_{z}$ interaction required for the simulation of more involved Hamiltonians such as quantum spin Hamiltonians. The geometric phase gates also allow the discussion of the $\hat{\sigma}_{z} \otimes \hat{\sigma}_{z}$ interaction in a familiar frame, while for the quantum Ising Hamiltonian a canonical transformation is introduced, which leads to a more involved dressed-state picture (see section 3.5).

The interaction according to the Hamiltonian can be implemented $[47,110,123]$ by stimulated-Raman transitions driven by two beams with wavevectors $\vec{k}_{1}, \vec{k}_{2}$ and difference frequency close to a (several) motional mode(s) (see figure $5(b))$. On average the differential ac Stark shift between the levels $|\downarrow\rangle$ and $|\uparrow\rangle$ caused by the two beams can be compensated by choosing appropriate polarizations of the beams. Still, on short timescales $\sim 2 \pi / \delta_{m}$ the ions experience a state-dependent force that leads to the above displacement in the phase spaces of the corresponding modes.

In the original implementation of the geometric phase gate [110] two ${ }^{9} \mathrm{Be}^{+}$ions are used. The state-dependent forces amount to $\vec{F}_{\downarrow}=-2 \vec{F}_{\uparrow}$. This means that the operators $\left(\alpha_{0} \hat{\mathbb{1}}^{(i)}+\right.$ $\left.\alpha_{3} \hat{\sigma}_{z}^{(i)}\right)$ have diagonal elements 1 and -2 , which is fulfilled for $\alpha_{0}=-1 / 2$ and $\alpha_{3}=3 / 2$. The effective wavevectors $\vec{k}_{\mathrm{I}}^{(1)}=\vec{k}_{\mathrm{I}}^{(2)}=\vec{k}_{1}-\vec{k}_{2}$ point along the axis of the linear trap and the laser beams are detuned by $\delta_{\mathrm{STR}}=2 \pi \times 26 \mathrm{kHz}$ from the stretch (STR) mode. The effect of the centre-ofmass $(\mathrm{COM})$ mode can be neglected $\left(\delta_{\mathrm{COM}} \approx 100 \delta_{\mathrm{STR}}\right)$. The ions are placed at the same phase of the stimulated-Raman interaction $\left(\varphi_{\mathrm{I}}^{(1)}=\varphi_{\mathrm{I}}^{(2)}=0\right)$.

For $t=T_{\mathrm{g}}=2 \pi / \delta_{\mathrm{STR}}$ the time evolution operator equation (3.33) simplifies to

$$
\begin{aligned}
& \hat{U}_{\mathrm{I}}^{\prime(\mathrm{LDR})(\mathrm{RWA})}\left(T_{\mathrm{g}}, 0\right) \approx \exp \left(-2 \pi \mathrm{i} \sum_{i=1}^{2} \sum_{j=1}^{2} \frac{(-1)^{i-j} \Omega_{\mathrm{I}}^{2} \eta_{\mathrm{STR}}^{2}}{\delta_{\mathrm{STR}}^{2}}\right. \\
& \left.\times\left[\alpha_{3}^{2} \hat{\sigma}_{z}^{(i)} \otimes \hat{\sigma}_{z}^{(j)}+\alpha_{0} \alpha_{3}\left(\hat{\sigma}_{z}^{(i)}+\hat{\sigma}_{z}^{(j)}\right)\right]\right)
\end{aligned}
$$

where we have used $\eta_{\mathrm{STR}}:=\eta_{\mathrm{STR}}^{(1)}=-\eta_{\mathrm{STR}}^{(2)}$ and neglected the global phase arising from the $\hat{\mathbb{1}}^{(i)} \otimes \hat{\mathbb{1}}^{(j)}$ terms. The sequence of the gate is similar to the one in figure 7 , but without the second displacement pulse $\hat{D}_{2}$. Ideally, the initial state $|\psi\rangle=|\downarrow \downarrow\rangle\left|n_{\mathrm{COM}}=0, n_{\mathrm{STR}}=0\right\rangle$ is rotated to $1 / 2(|\downarrow \downarrow\rangle+|\downarrow \uparrow\rangle+|\uparrow \downarrow\rangle+|\uparrow \uparrow\rangle)\left|n_{\mathrm{COM}}=0, n_{\mathrm{STR}}=0\right\rangle$ by the first $\hat{R}(\pi / 2, \pi / 2)$ pulse (the phase $\varphi$ of the first pulse can be chosen arbitrarily). The only non-vanishing contributions arise from the $\hat{\sigma}_{z} \otimes \hat{\sigma}_{z}$ terms for the $|\downarrow \uparrow\rangle$ and $|\uparrow \downarrow\rangle$ states, which gain a geometric phase

$$
\Phi_{\mathrm{STR} \downarrow \uparrow / \uparrow \downarrow}=-2 \pi \times 4 \frac{\Omega_{\mathrm{I}}^{2} \eta_{\mathrm{STR}}^{2}}{\delta_{\mathrm{STR}}^{2}} \alpha_{3}^{2} .
$$

(a)

(b)

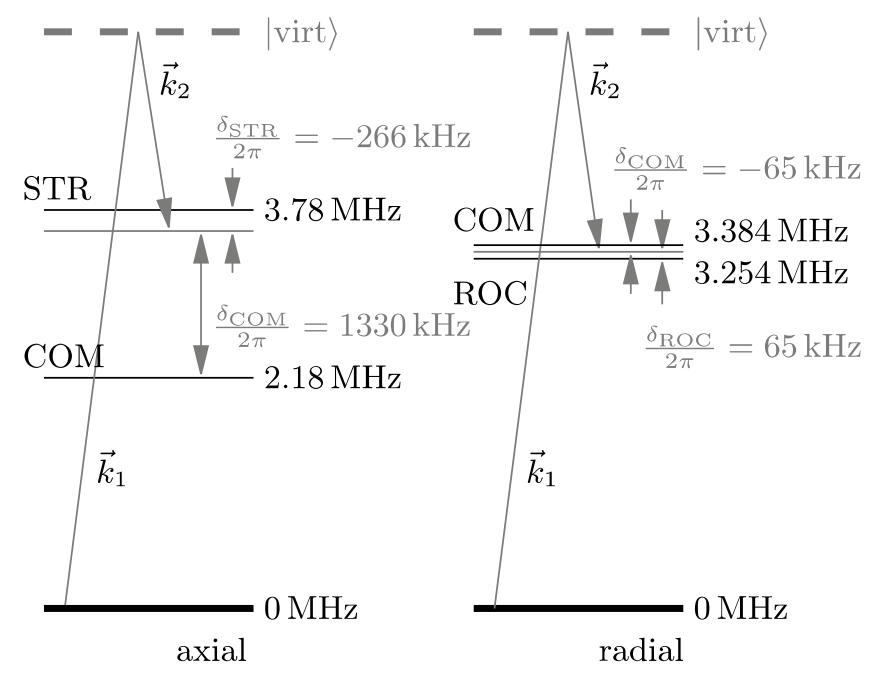

Figure 6. Comparison between parameters of geometric phase gates [110] with two ions using the axial motional modes and radial motional modes. (a) The parameters correspond to the gate from [123]. The axial centre-of-mass (COM) and stretch (STR) mode have a large frequency difference $(2 \pi \times 1.6 \mathrm{MHz})$. The detuning of the Raman beams from the STR mode amounts to $\delta_{\mathrm{STR}}=-2 \pi \times 266 \mathrm{MHz}$. That is why the main contribution to the differential geometric phase between $|\downarrow \downarrow\rangle /|\uparrow \uparrow \uparrow\rangle$ and $|\downarrow \uparrow\rangle /|\uparrow \downarrow\rangle$ is due to a (single) loop in the phase space of the STR mode. However, as already suggested in [110], the detuning from the COM mode is chosen to be an integer multiple of the detuning from the STR mode $\left(\delta_{\mathrm{COM}}=-5 \times \delta_{\mathrm{STR}}\right)$. Hence, there is no entanglement left between the electronic and motional modes at the gate duration

$T_{\mathrm{g}}=\left|2 \pi / \delta_{\mathrm{STR}}\right|=3.75 \mu \mathrm{s}$. (Note that the spin-echo sequence is not included in $T_{\mathrm{g}}$.) (b) The parameters correspond to a phase gate on two of the radial motional modes. The radial centre-of-mass (COM) and rocking (ROC) mode have a comparatively small frequency difference of only $2 \pi \times 130 \mathrm{kHz}$. The detunings from both modes are chosen to have the same absolute values resulting in (approximately) equal contributions to the acquired geometric phase from both modes. The gate duration according to the original implementation would amount to $T_{\mathrm{g}}=\left|2 \pi / \delta_{\mathrm{STR}}\right|=15.4 \mu \mathrm{s}$. As the displacement pulse is repeated in the second gap of the spin-echo sequence (compare figure 7) to cancel dynamic phases (compare [118] and see text), the duration increases by an additional factor of two.

By choosing appropriate beam intensities and thus $\Omega_{\mathrm{I}}$, these phases equal $\Phi_{\mathrm{STR} \downarrow \uparrow / \uparrow \downarrow}=-\pi / 2$. The subsequent $\hat{R}(\pi, \pi / 2)$ and $\hat{R}(\pi / 2, \pi / 2)$ pulses lead to the final Bell state $|\tilde{\psi}\rangle=$ $1 / \sqrt{2}(|\downarrow \downarrow\rangle+i|\uparrow \uparrow\rangle)$, which is achieved experimentally with a fidelity of $F=97 \%$ [110].

A similar implementation of the geometric phase gate is reported in [123] based on two ${ }^{25} \mathrm{Mg}^{+}$ions. The statedependent forces amount to $\vec{F}_{\downarrow}=-3 / 2 \vec{F}_{\uparrow}\left(\alpha_{0}=-1 / 4\right.$ and $\left.\alpha_{3}=5 / 4\right)$. Furthermore, the detuning from the STR mode amounts to $\delta_{\mathrm{STR}}=-2 \pi \times 266 \mathrm{kHz}$ and simultaneously the detuning from the COM mode $\delta_{\mathrm{COM}}=-2 \pi \times 1330 \mathrm{kHz}$ (compare figure 6(a)). Hence, the effect of the COM mode is also exploited for the gate. As the detuning from the COM mode is chosen to be an integer multiple of the detuning from the STR mode $\left(\delta_{\mathrm{COM}}=-5 \delta_{\mathrm{STR}}\right)$, the first exponential in equation (3.33) still becomes unity for the gate duration of $T_{\mathrm{g}}=\left|2 \pi / \delta_{\mathrm{STR}}\right|$. (In other words, all circular trajectories in 
all phase spaces return to their initial position for $T_{\mathrm{g}}$.) As a result, there is no entanglement left between the electronic and motional states.

Analogous to equation (3.35), but considering $\delta_{\mathrm{STR}}<0$ and $\delta_{\mathrm{COM}}>0$ for the detunings and $\eta_{\mathrm{COM}}^{(1)}=\eta_{\mathrm{COM}}^{(2)}$ for the Lamb-Dicke parameters of the COM mode, the geometric phases yield

$$
\begin{aligned}
& \Phi_{\mathrm{STR} \downarrow \uparrow / \uparrow \downarrow}=2 \pi \times 4 \frac{\Omega_{\mathrm{I}}^{2} \eta_{\mathrm{STR}}^{2}}{\delta_{\mathrm{STR}}^{2}} \alpha_{3}^{2}, \\
& \Phi_{\mathrm{COM} \downarrow \downarrow / \uparrow \uparrow}=-2 \pi\left|\frac{\delta_{\mathrm{COM}}}{\delta_{\mathrm{STR}}}\right| \times 4 \frac{\Omega_{\mathrm{I}}^{2} \eta_{\mathrm{COM}}^{2}}{\delta_{\mathrm{COM}}^{2}} \alpha_{3}^{2} .
\end{aligned}
$$

By adjusting the beam intensities appropriately the differential phase between $|\downarrow \downarrow\rangle /|\uparrow \uparrow\rangle$ and $|\downarrow \uparrow\rangle /|\uparrow \downarrow\rangle$ can be adjusted to fulfil $\Phi_{\mathrm{STR} \downarrow \uparrow / \uparrow \downarrow}-\Phi_{\mathrm{COM} \downarrow \downarrow / \uparrow \uparrow}=\pi / 2$. As $\Phi_{\mathrm{STR} \downarrow \uparrow / \uparrow \downarrow}$ has the opposite sign compared with $\Phi_{\mathrm{COM} \downarrow \downarrow / \uparrow \uparrow}$, the geometric phase gate makes use of two motional modes simultaneously.

However, some of the dynamic phases from the COM mode do not vanish:

$$
\tilde{\Phi}_{\mathrm{COM} \downarrow \downarrow / \uparrow \uparrow}= \pm 2 \pi\left|\frac{\delta_{\mathrm{COM}}}{\delta_{\mathrm{STR}}}\right| \times 8 \frac{\Omega_{\mathrm{I}}^{2} \eta_{\mathrm{COM}}^{2}}{\delta_{\mathrm{COM}}^{2}} \alpha_{0} \alpha_{3} .
$$

These phases have an absolute value of $2 \alpha_{0} / \alpha_{3}$ of the geometric phase from the COM mode and lead to a small deviation from the ideal state at the end of the gate.

Compared with the original implementation in [110], the gate is speeded up by approximately a factor of 10 and the fidelity $F$ for the Bell state exceeds $95 \%$. (Note that the duration of the spin-echo sequence is not included in $T_{\mathrm{g}}$, because its rotations could be much faster and empty gaps can in principle be removed.)

The radial motional modes are interesting, because they are similar to the normal modes in systems of individual traps for each ion (compare section 6), which are promising candidates for scalable systems in quantum simulations. To investigate the differences between the axial and radial modes of motion the geometric phase gate with ${ }^{25} \mathrm{Mg}^{+}$is performed on a pair of radial modes (see also [122] for a Mølmer-Sørensen gate performed on the radial modes).

The detunings from the COM and ROC mode (abbreviation for 'rocking' mode, the equivalent to the STR mode in terms of the axial motional modes) are chosen to have the same absolute values $\delta_{\mathrm{COM}}=-\delta_{\mathrm{ROC}}=2 \pi \times 65 \mathrm{kHz}$ (see figure 6). The geometric phases acquired on each motional mode are basically the same as in equation (3.36), where 'STR' has to be replaced by 'ROC', and equation (3.37). (However, the signs change due to a change of the signs of the detunings.) The contributions to the total differential geometric phase between $|\downarrow \downarrow\rangle /|\uparrow \uparrow\rangle$ and $|\downarrow \uparrow\rangle /|\uparrow \downarrow\rangle$ due to the COM and ROC mode are (approximately) equal now. However, the dynamic phase (analogous to equation (3.38)) arising from the COM mode can no longer be neglected.

The pulse scheme of the geometric phase gate is modified by adding a second displacement pulse in the second gap of the spin-echo sequence (see figure 7 and compare [118]). The intensities of the beams are now adjusted for differential geometric phases due to each displacement pulse of

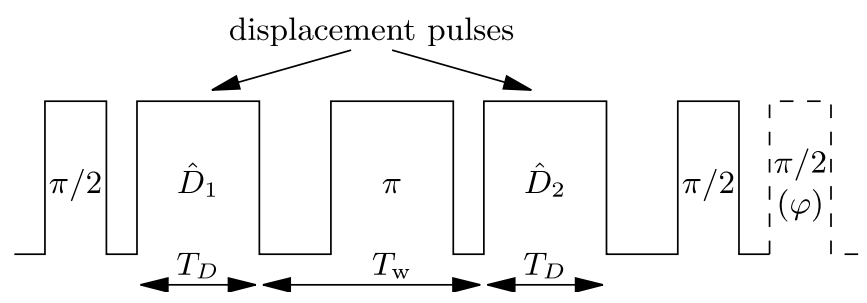

Figure 7. Pulse scheme of the geometric phase gate. It consists of a spin-echo sequence $(\hat{R}(\pi / 2, \pi / 2), \hat{R}(\pi, \pi / 2), \hat{R}(\pi / 2, \pi / 2)$ pulses $)$ with a displacement pulse (labelled ' $\hat{D}_{1}$ ') in the first gap of the spin-echo sequence for the original implementation of the phase gate [110]. For the gate on the radial modes of motion a second displacement pulse (labelled ' $\hat{D}_{2}$ ') is introduced to cancel dynamic phases from $\hat{D}_{1}$ due to different absolute values of the forces on $|\uparrow\rangle$ and $|\downarrow\rangle$ (compare [118]). For all gates the duration of each displacement pulse is chosen to be $T_{D}=\left|2 \pi / \delta_{\mathrm{STR} / \mathrm{ROC}}\right|$ such that each displacement pulse leads to a closed loop in each phase space. Hence, the total gate duration amounts to $T_{\mathrm{g}}=T_{D}$ for the original implementation and $T_{\mathrm{g}}=2 T_{D}$ for the gate on the radial modes of motion. (Note that the spin-echo sequence is not included in $T_{\mathrm{g}}$.) The dashed $\hat{R}(\pi / 2, \pi / 2+\varphi)$ analysis pulse is added for the measurement of the gate fidelity (see figure 9 ).

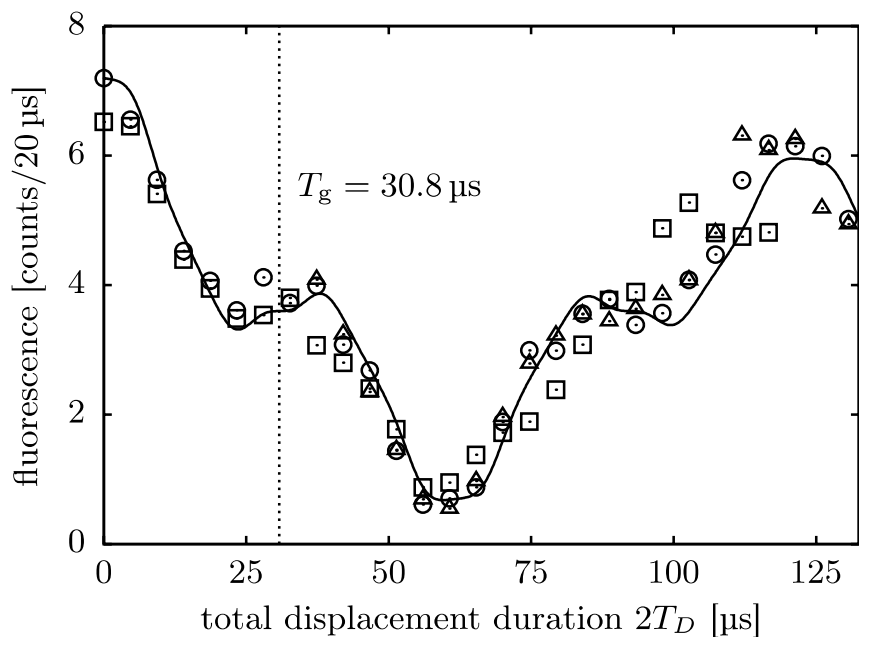

Figure 8. Total fluorescence from the two ions as a function of the total displacement duration $2 T_{D}$ (compare figure 7 ). The detected fluorescence signal from both ions amounts to approximately 7 counts $/ 20 \mu$ s for state $|\downarrow \downarrow\rangle$ and close to zero for $|\uparrow \uparrow\rangle$. The duration between the displacement pulses is chosen to be $T_{\mathrm{w}}=\left|2 \pi / \delta_{\mathrm{COM} / \mathrm{ROC}}\right|$ in the experiment (compare figure 7). At $T_{\mathrm{g}} \approx 30.8 \mu$ s the state $|\psi\rangle \approx(|\uparrow \uparrow\rangle+\mathrm{i}|\downarrow \downarrow\rangle)\left|n_{\mathrm{COM}}=0, n_{\mathrm{ROC}}=0\right\rangle$ is prepared. Each data point represents the average of 400 measurements (squares and triangles) and 200 measurements (circles), respectively. The statistical errors are on the order of the size of the symbols. The curve is based on a fit of the time evolution of equation (3.33) with an additional empirical exponential decay to mimic decoherence effects. The only fit parameters are the fluorescence for $|\downarrow \downarrow\rangle$ amounting to 7.2 counts $/ 20 \mu$ s and the decay constant $\tau \approx 290 \mu \mathrm{s}$. The gate serves as an experimental reference for the isolated interaction strength and is not optimized to provide the highest gate fidelity.

$\Phi_{\mathrm{COM} \downarrow \downarrow / \uparrow \uparrow}-\Phi_{\mathrm{ROC} \downarrow \uparrow / \uparrow \downarrow}=\pi / 4$. While the geometric phases of both displacement pulses add up to $\pi / 2$, the dynamic phases cancel each other, as the $\pi$ pulse of the spin-echo sequence interchanges $|\downarrow \downarrow\rangle \leftrightarrow|\uparrow \uparrow\rangle$ (and $|\downarrow \uparrow\rangle \leftrightarrow|\uparrow \downarrow\rangle$ ). Additionally, the more symmetric pulse scheme enhances the robustness 


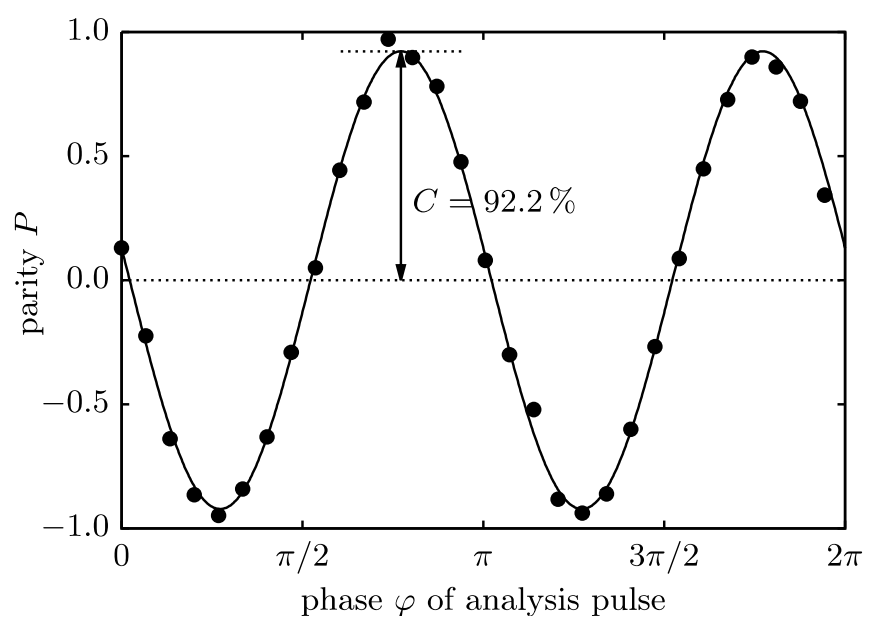

Figure 9. Parity measurement after the geometric phase gate on two radial modes of motion with two ions. The parity is defined as $P:=P_{\downarrow \downarrow}+P_{\uparrow \uparrow}-\left(P_{\uparrow \downarrow}+P_{\downarrow \uparrow}\right)$, where $P_{s, s^{\prime}}$ denotes the population of the electronic state $\left|s, s^{\prime}\right\rangle$ with $s, s^{\prime} \in\{\downarrow, \uparrow\}$. It is measured as a function of the phase $\varphi$ of the analysis pulse $\hat{R}(\pi / 2, \pi / 2+\varphi)$ (compare figure 7). Each data point represents the mean of 2500 measurements. The contrast $C=92.2 \%$ is determined from the fitted curve. Considering the populations $P_{\downarrow \downarrow}+P_{\uparrow \uparrow}>98 \%$ for the entangled state we obtain a Bell state fidelity $F>95 \%$.

of the gate against uncompensated differential ac Stark shifts between $|\downarrow\rangle$ and $|\uparrow\rangle$.

The total fluorescence from the two ions as a function of the total duration of the displacements $2 T_{D}$ is shown in figure 8 . The gate duration due to the smaller detunings and the second displacement pulse is more than a factor of eight longer than for the gate in [123]. Still, the fidelity exceeds $95 \%$ (see figure 9).

\subsection{Quantum Ising Hamiltonian}

Above we have introduced $\hat{\sigma}_{z} \otimes \hat{\sigma}_{z}$ interactions that are used in quantum gates. In the following we will present a slightly different approach, in which Ising spin-spin interactions are continuously induced by means of optical forces.

The spin-spin interaction as proposed in [21] and experimentally realized in the simulation of a quantum Ising Hamiltonian in [47] is identical to the interaction $\hat{\mathcal{H}}_{\mathrm{I}}$ described in section 3.3. (Note that a similar proposal involving the same mathematics is given in [23].) However, the quantum Ising Hamiltonian contains an additional (simulated) magnetic field pointing in the $x$-direction. We will adapt our notation in this section and split the total interaction Hamiltonian into the following terms: $\hat{\mathcal{H}}_{\mathrm{S}}$ denotes the term that generates the spin-spin interaction and $\hat{\mathcal{H}}_{\mathrm{M}}$ denotes the term leading to the simulated magnetic field. The index ' $\mathrm{I}$ ' of the frequencies $\Omega_{\mathrm{I}}$ and $\omega_{\mathrm{I}}$, etc is changed to ' $\mathrm{S}$ ' or ' $\mathrm{M}$ ' accordingly in the respective terms. The complete interaction is described by the Hamiltonian $\hat{\mathcal{H}}_{\mathrm{I}}=\hat{\mathcal{H}}_{\mathrm{S}}+\hat{\mathcal{H}}_{\mathrm{M}}$. In the following, we will first derive the spin-spin interaction Hamiltonian from $\hat{\mathcal{H}}_{\mathrm{S}}$ focusing on an Ising interaction $\left(\hat{\sigma}_{z} \otimes \hat{\sigma}_{z}\right.$ only). Afterwards, we will discuss the magnetic field term $\hat{\mathcal{H}}_{\mathrm{M}}$ and its effect.

The derivation of the quantum Ising Hamiltonian [21, 124] involves a slightly different interaction picture compared with section 3.1 by substituting $\hat{\mathcal{H}}_{0}$ with $\hat{\mathcal{H}}_{\varnothing}$ :

$$
\begin{aligned}
\hat{\mathcal{H}}_{0} & =\hat{\mathcal{H}}_{\mathrm{e}}+\hat{\mathcal{H}}_{\mathrm{m}} \\
= & \underbrace{\hat{\mathcal{H}}_{\mathrm{e}}+\sum_{m=1}^{3 N} \hbar \omega_{\mathrm{s}} \hat{a}_{m}^{\dagger} \hat{a}_{m}}_{=: \hat{\mathcal{H}}_{\emptyset}} \underbrace{-\sum_{m=1}^{3 N} \hbar \delta_{m} \hat{a}_{m}^{\dagger} \hat{a}_{m}}_{=: \hat{\mathcal{H}}_{\delta}} .
\end{aligned}
$$

The term $\hat{\mathcal{H}}_{\delta}$ is added to the interaction Hamiltonian.

To retrieve the representation of $\hat{\mathcal{H}}_{\mathrm{S}}$ in the newly defined interaction picture,

$$
\hat{\mathcal{H}}_{\mathrm{S}}^{\prime}:=\hat{U}_{\emptyset}^{\dagger} \hat{\mathcal{H}}_{\mathrm{S}} \hat{U}_{\emptyset} \quad \text { with } \quad \hat{U}_{\emptyset}:=\mathrm{e}^{-\mathrm{i} \hat{\mathcal{H}}_{\emptyset} t / \hbar}
$$

we adapt the calculations from sections 3.1 and 3.3 accordingly: the frequencies in the transformation equation (3.14) are changed to $\omega_{m} \rightarrow \omega_{\mathrm{S}}$. As a result, the substitution $\mathrm{e}^{ \pm \mathrm{i} \omega_{m} t} \rightarrow \mathrm{e}^{ \pm \mathrm{i} \omega_{\mathrm{S}} t}$ has to be applied to equation (3.15) (and subsequent equations) and $\mathrm{e}^{ \pm \mathrm{i} \delta_{m} t} \rightarrow 1$ to equation (3.32). Hence, $\hat{\mathcal{H}}_{\mathrm{S}}$ reads in the new interaction picture (including the expansion to first order in the Lamb-Dicke parameters and the RWA):

$$
\begin{gathered}
\hat{\mathcal{H}}_{\mathrm{S}}^{\prime(\mathrm{LDR})(\mathrm{RWA})}=\sum_{i=1}^{N} \sum_{m=1}^{3 N} \mathrm{i} \hbar \Omega_{\mathrm{S}}^{(i)} \eta_{m}^{(i)} \mathrm{e}^{\mathrm{i} \varphi_{\mathrm{S}}^{(i)}} \hat{a}_{m}^{\dagger} \\
\times\left(\alpha_{0} \hat{\mathbb{1}}^{(i)}+\alpha_{3} \hat{\sigma}_{z}^{(i)}\right)+\text { h.c. }
\end{gathered}
$$

However, the full Hamiltonian in the interaction picture now also involves

$$
\hat{\mathcal{H}}_{\delta}^{\prime}=\hat{U}_{\emptyset}^{\dagger} \hat{\mathcal{H}}_{\delta} \hat{U}_{\varnothing}=\hat{\mathcal{H}}_{\delta},
$$

where the transformation is the identity, because trivially $\left[\hat{\mathcal{H}}_{\varnothing}, \hat{\mathcal{H}}_{\delta}\right]=0$.

To gain the form of a spin-spin interaction, we apply a canonical transformation (compare [21]) to the Hamiltonian. We want to note that an adiabatic elimination of phonons would yield the same effective description of the system; however, the canonical transformation provides a systematic method to also calculate the corrections to the ideal quantum Ising model:

$$
\hat{\mathcal{H}}_{\mathrm{S}}^{\prime \prime(\mathrm{LDR})(\mathrm{RWA})}+\hat{\mathcal{H}}_{\delta}^{\prime \prime}:=\hat{U}_{\mathrm{c}}\left(\hat{\mathcal{H}}_{\mathrm{S}}^{\prime(\mathrm{LDR})(\mathrm{RWA})}+\hat{\mathcal{H}}_{\delta}\right) \hat{U}_{\mathrm{c}}^{\dagger}
$$

with

$$
\hat{U}_{\mathrm{c}}:=\exp \left(-\sum_{i=1}^{N} \sum_{m=1}^{3 N} \frac{1}{\hbar \delta_{m}}\left[\hat{\xi}_{m}^{(i)} \hat{a}_{m}^{\dagger}-\hat{\xi}_{m}^{(i) \dagger} \hat{a}_{m}\right]\right)
$$

and

$$
\hat{\xi}_{m}^{(i)}:=\mathrm{i} \hbar \Omega_{\mathrm{S}}^{(i)} \eta_{m}^{(i)} \mathrm{e}^{\mathrm{i} \varphi_{\mathrm{S}}^{(i)}}\left(\alpha_{0} \hat{\mathbb{1}}^{(i)}+\alpha_{3} \hat{\sigma}_{z}^{(i)}\right) .
$$

Using the calculations from appendix $G$, the transformed Hamiltonian reads

$$
\begin{aligned}
\hat{\mathcal{H}}_{\mathrm{S}}^{\prime \prime(\mathrm{LDR})(\mathrm{RWA})}+\hat{\mathcal{H}}_{\delta}^{\prime \prime}=\sum_{i=1}^{N} \sum_{j=1}^{N} \sum_{m=1}^{3 N} \frac{1}{\hbar \delta_{m}} \hat{\xi}_{m}^{(i)} \otimes \hat{\xi}_{m}^{(j) \dagger}+\hat{\mathcal{H}}_{\delta} \\
=\hbar \sum_{i=1}^{N} \sum_{j=1}^{N} \sum_{m=1}^{3 N} \frac{\Omega_{\mathrm{S}}^{(i)} \Omega_{\mathrm{S}}^{(j)} \eta_{m}^{(i)} \eta_{m}^{(j)}}{\delta_{m}} \mathrm{e}^{\mathrm{i}\left(\varphi_{\mathrm{S}}^{(i)}-\varphi_{\mathrm{S}}^{(j)}\right)} \\
\times\left(\alpha_{0} \hat{\mathbb{1}}^{(i)}+\alpha_{3} \hat{\sigma}_{z}^{(i)}\right) \otimes\left(\alpha_{0} \hat{\mathbb{1}}^{(j)}+\alpha_{3} \hat{\sigma}_{z}^{(j)}\right)+\hat{\mathcal{H}}_{\delta} .
\end{aligned}
$$


The Hamiltonian can be expanded into a pure $\hat{\sigma}_{z} \otimes \hat{\sigma}_{z}$ interaction, a 'bias' term with $\hat{\sigma}_{z}$ interaction, and a constant term that can be neglected. The 'bias' term acts as a longitudinal magnetic field and leads to a deviation from the quantum Ising model. At first glance, this is not desired and it will be treated as an error in the following discussion. However, by including a 'bias' term in a controlled way we could also explore an extended phase diagram with the longitudinal field as an additional parameter.

We want to stress the similarity between the spinspin interaction according to equation (3.46) and the $\hat{\sigma}_{z} \otimes$ $\hat{\sigma}_{z}$ interaction discussed in section 3.3. The canonical transformation has the form of a displacement operator and looks very similar to the first exponential function in equation (3.33) (except for the time dependence of the latter). The similarity to the geometric phase term in equation (3.33) can be best seen comparing the time evolution operators. As the Hamiltonian in equation (3.46) is time-independent, the time evolution simply reads

$$
\begin{aligned}
\hat{U}_{\mathrm{S}}^{\prime \prime(\mathrm{LDR})(\mathrm{RWA})}\left(t, t_{0}\right) \times \hat{U}_{\delta}\left(t, t_{0}\right) & \\
= & \exp \left(-\mathrm{i} \sum_{i=1}^{N} \sum_{j=1}^{N} \sum_{m=1}^{3 N} \frac{\Omega_{\mathrm{S}}^{(i)} \Omega_{\mathrm{S}}^{(j)} \eta_{m}^{(i)} \eta_{m}^{(j)}}{\delta_{m}^{2}}\left(\alpha_{0} \hat{\mathbb{1}}^{(i)}+\alpha_{3} \hat{\sigma}_{z}^{(i)}\right)\right. \\
& \left.\otimes\left(\alpha_{0} \hat{\mathbb{1}}^{(j)}+\alpha_{3} \hat{\sigma}_{z}^{(j)}\right) \times \delta_{m}\left(t-t_{0}\right) \mathrm{e}^{\mathrm{i}\left(\varphi_{\mathrm{S}}^{(i)}-\varphi_{\mathrm{S}}^{(j)}\right)}\right) \\
& \times \exp \left(\mathrm{i} \sum_{m=1}^{3 N} \delta_{m}\left(t-t_{0}\right) \hat{a}_{m}^{\dagger} \hat{a}_{m}\right) .
\end{aligned}
$$

Before we can apply the easier time evolution of equation (3.47), in which electronic states are decoupled from motional states, the state vector $|\psi\rangle$ has to be transformed from the original picture to $|\psi\rangle^{\prime \prime}:=\hat{U}_{\mathrm{c}}|\psi\rangle$. As $\hat{U}_{\mathrm{c}}$ depends on the electronic state, the transformation will in general lead to an entangled state and the canonical transformation can be interpreted as dressed-state picture (electronic states 'dressed' with motional states). As the states for the simulation of the quantum Ising Hamiltonian are prepared in the original (undressed) picture, but the Hamiltonian acts in the dressed picture, an error is introduced into the simulation (see, for example, [125]). However, as long as the effect due to $\hat{U}_{\mathrm{c}}$ is small $\left(\left|\Omega_{\mathrm{S}}^{(i)} \eta_{m}^{(i)} \alpha_{l} / \delta_{m}\right| \ll 1\right)$, we can use the approximation $|\psi\rangle^{\prime \prime} \approx|\psi\rangle$. In terms of the geometric phase gate this corresponds to the case when the circles in phase space are small and the entanglement between electronic and motional states can be neglected at any time.

The same holds for the measurements of observables: they are performed in the original (undressed) picture, in which electronic states are entangled with the motional states, and in general a further error is introduced in the simulation. However, the measurement of the states is typically insensitive to the motional states and involves a projection to one of the electronic states, for example, the $|\downarrow\rangle$ state: $\hat{P}^{(i)}:=|\downarrow\rangle^{(i)}\left\langle\left.\downarrow\right|^{(i)}\right.$. As $\left[\hat{U}_{\mathrm{c}}, \hat{P}^{(i)}\right]=0$, the projector does not change under the canonical transformation and the readout of $\hat{\sigma}_{z}$ eigenstates (without any rotations of the bases applied beforehand) does not introduce further errors.
The form of the 'bias' term proportional to $\hat{\sigma}_{z}$ can be simplified in the case of a linear Paul trap with equal Rabi frequencies $\Omega_{\mathrm{S}}^{(i)}$ and equal phases $\varphi_{\mathrm{S}}^{(i)}$ for all ions: The sum over $j$ extends over the Lamb-Dicke parameters $\eta_{m}^{(j)}$ only. This sum is non-zero only for centre-of-mass modes, for which the $\eta_{m}^{(j)}$ are additionally independent of the site $j$. Hence, the three sums simplify to a sum over $\hat{\sigma}_{z}^{(i)}$ with constant prefactor [21]:

$$
2 \Omega_{\mathrm{S}}^{2} \hbar N \alpha_{0} \alpha_{3}\left(\sum_{m \in\{\mathrm{c} . \mathrm{m} .\}} \frac{\eta_{m}^{2}}{\delta_{m}}\right) \sum_{i=1}^{N} \hat{\sigma}_{z}^{(i)} .
$$

However, this simplification does not necessarily hold for twodimensional arrays of individual traps for each ion.

In the following, we will discuss the magnetic field term, which originates from a $\hat{\sigma}_{x}$ interaction described by equation (3.17) (with $\alpha_{1}=1$ and $\alpha_{2}=0$ ). In principle, we have to apply the substitution $\mathrm{e}^{ \pm \mathrm{i} \omega_{m} t} \rightarrow \mathrm{e}^{ \pm \mathrm{i} \omega_{\mathrm{s}} t}$ due to the new interaction picture here, too. However, we consider a magnetic field term without motional dependence in the following $\left(\eta_{m}^{(i)}=0\right.$, compare section 3.2) and thus the terms containing the motional creation/annihilation operators vanish:

$$
\hat{\mathcal{H}}_{\mathrm{M}}^{\prime(\mathrm{RWA})}=\sum_{i=1}^{N} \frac{\hbar}{2} \Omega_{\mathrm{M}}^{(i)} \mathrm{e}^{\mathrm{i}\left(-\left(\omega_{\mathrm{M}}-\omega_{\uparrow \downarrow}\right) t+\varphi_{\mathrm{M}}^{(i)}\right)} \hat{\sigma}_{+}^{(i)}+\text { h.c. }
$$

The canonical transformation can be rewritten as

$$
\hat{U}_{\mathrm{c}}=\exp \left(\mathrm{i} \sum_{i=1}^{N} \hat{h}^{(i)}\left(\alpha_{0} \hat{\mathbb{1}}^{(i)}+\alpha_{3} \hat{\sigma}_{z}^{(i)}\right)\right)
$$

with the Hermitian operator

$$
\hat{h}^{(i)}:=\sum_{m=1}^{3 N}\left[\zeta_{m}^{(i)} \hat{a}_{m}^{\dagger}+\zeta_{m}^{(i) \dagger} \hat{a}_{m}\right]
$$

and

$$
\zeta_{m}^{(i)}:=-\frac{\hbar \Omega_{\mathrm{S}}^{(i)} \eta_{m}^{(i)} \mathrm{e}^{\mathrm{i} \varphi_{\mathrm{S}}^{(i)}}}{\hbar \delta_{m}}
$$

Trivially, the commutator $\left[\hat{h}^{(i)}, \hat{\sigma}_{+}^{(i)}\right]=0$. The canonical transformation of $\hat{\mathcal{H}}_{\mathrm{M}}^{\prime(\mathrm{RWA})}$ (see equation (3.49)) is thus equivalent to a transformation of the $\hat{\sigma}_{ \pm}^{(i)}$ operator as in equations (B.16) and (B.17):

$$
\begin{aligned}
& \hat{\mathcal{H}}_{\mathrm{M}}^{\prime \prime(\mathrm{RWA})}=\hat{U}_{\mathrm{c}} \hat{\mathcal{H}}_{\mathrm{M}}^{\prime(\mathrm{RWA})} \hat{U}_{\mathrm{c}}^{\dagger} \\
& =\sum_{i=1}^{N} \frac{\hbar}{2} \Omega_{\mathrm{M}}^{(i)} \mathrm{e}^{\mathrm{i}\left(-\left(\omega_{\mathrm{M}}-\omega_{\uparrow \downarrow}\right) t+\varphi_{\mathrm{M}}^{(i)}\right)} \mathrm{e}^{2 \mathrm{i} \alpha_{3} \hat{h}^{(i)}} \hat{\sigma}_{+}^{(i)}+\text { h.c. }
\end{aligned}
$$

The expansion to first order in $\zeta_{m}^{(i)}$ (and thus to first order in $\left.\hat{\eta}_{m}^{(i)}\right)$ yields

$$
\begin{aligned}
\hat{\mathcal{H}}_{\mathrm{M}}^{\prime \prime(\mathrm{RWA})} & \approx \sum_{i=1}^{N} \frac{\hbar}{2} \Omega_{\mathrm{M}}^{(i)} \mathrm{e}^{\mathrm{i}\left(-\left(\omega_{\mathrm{M}}-\omega_{\uparrow \downarrow}\right) t+\varphi_{\mathrm{M}}^{(i)}\right)} \\
& \times\left(1+2 \mathrm{i} \alpha_{3} \sum_{m=1}^{3 N}\left[\zeta_{m}^{(i)} \hat{a}_{m}^{\dagger}+\zeta_{m}^{(i) \dagger} \hat{a}_{m}\right]\right) \hat{\sigma}_{+}^{(i)}+\text { h.c. } \\
= & : \hat{\mathcal{H}}_{\mathrm{M}}^{\prime(\mathrm{RWA})}+\hat{\mathcal{H}}_{\mathrm{E}}^{\prime \prime}
\end{aligned}
$$


The magnetic field term after the canonical transformation deviates to order $\Omega_{\mathrm{S}}^{(i)} \eta_{m}^{(i)} \alpha_{3} / \delta_{m}$ due to $\hat{\mathcal{H}}_{\mathrm{E}}^{\prime \prime}$ from the pure $\hat{\sigma}_{x}$ interaction $\hat{\mathcal{H}}_{\mathrm{M}}^{\prime(\mathrm{RWA})}$. This introduces an additional error in the simulation. If the condition $\left|\Omega_{\mathrm{S}}^{(i)} \eta_{m}^{(i)} \alpha_{l} / \delta_{m}\right| \ll 1$ is met, it can be small or even negligible and we, effectively, will obtain the desired magnetic field term.

To summarize, the complete Hamiltonian is obtained by adding equation (3.46) and equation (3.54). It consists of a spin-spin interaction term and a simulated magnetic field pointing in the $x$-direction, which add up to the ideal quantum Ising Hamiltonian. Assuming a resonant interaction for the simulated magnetic field $\left(\omega_{\mathrm{M}}-\omega_{\uparrow \downarrow}=0\right)$ and neglecting the phases $\left(\varphi_{\mathrm{S}}^{(i)}=\varphi_{\mathrm{M}}^{(i)}=0\right)$, the quantum Ising part can be written as

$$
\begin{aligned}
\hat{\mathcal{H}}_{\text {QIsing }} & :=\hat{\mathcal{H}}_{\mathrm{B}}+\hat{\mathcal{H}}_{\mathrm{J}} \\
& =\sum_{i=1}^{N} B_{x}^{(i)} \hat{\sigma}_{x}^{(i)}+\sum_{i=1}^{N} \sum_{\substack{j=1 \\
j \neq i}}^{N} J^{(i, j)} \hat{\sigma}_{z}^{(i)} \otimes \hat{\sigma}_{z}^{(j)},
\end{aligned}
$$

where

$$
B_{x}^{(i)}:=\hbar \Omega_{\mathrm{M}}^{(i)} \quad \text { and } \quad J^{(i, j)}:=\hbar \sum_{m=1}^{3 N} \frac{\Omega_{\mathrm{S}}^{(i)} \Omega_{\mathrm{S}}^{(j)} \eta_{m}^{(i)} \eta_{m}^{(j)}}{\delta_{m}} \alpha_{3}^{2} .
$$

(Note that the superscripts of $B_{x}^{(i)}$ and $J^{(i, j)}$ indicating the site will be omitted in the following sections, if the interaction strengths for all ions are equal.)

Depending on the sign of the detunings $\delta_{m}$, the effective spin-spin interaction can be of ferromagnetic $\left(J^{(i, j)}<0\right)$ or antiferromagnetic $\left(J^{(i, j)}>0\right)$ nature. The range and spatial structure of the spin-spin interactions can be partially controlled by choosing the absolute values of the detunings $\delta_{m}$. There are two different limits in which the interaction can be shaped in a very controlled way. On the one hand, if the laser is tuned close to the frequency of a given mode $m^{\prime}\left(\left|\delta_{m \neq m^{\prime}}\right| \gg\right.$ $\left.\left|\delta_{m^{\prime}}\right| \forall m\right)$, then $J^{(i, j)}$ is a long-range interaction, whose spatial dependence is governed by the phonon wavefunction corresponding to $m^{\prime}$, as can be seen directly in equation (3.56). On the other hand, if all the motional modes contribute to the spin-spin coupling $\left(\left|\delta_{m}-\delta_{m^{\prime}}\right| \ll\left|\delta_{m^{\prime}}\right| \forall m, m^{\prime}\right)$, the interaction shows a power-law decay $J^{(i, j)} \propto 1 /|i-j|^{3}[21,124]$. This can be understood by the partial interference between the contributions from each mode's wavefunction.

Furthermore, the freedom in the choice of the coupling strengths $\Omega_{\mathrm{S}}^{(i)}$ for the individual ions as well as the trapping geometry and laser direction going into $\eta_{m}^{(i)}$ allows us to shape the individual coupling strengths $J^{(i, j)}$. This opens up the possibility of simulating a rich variety of models beyond the quantum Ising Hamiltonian, such as spin-frustrated systems [48] (see section 5.1). An illustration of the normal modes of motion and an example of the effective spin-spin couplings in a surface-electrode trap similar to the traps discussed in section 6 is given in figure 10 .

In addition to the quantum Ising part discussed above the complete Hamiltonian consists of the following terms that

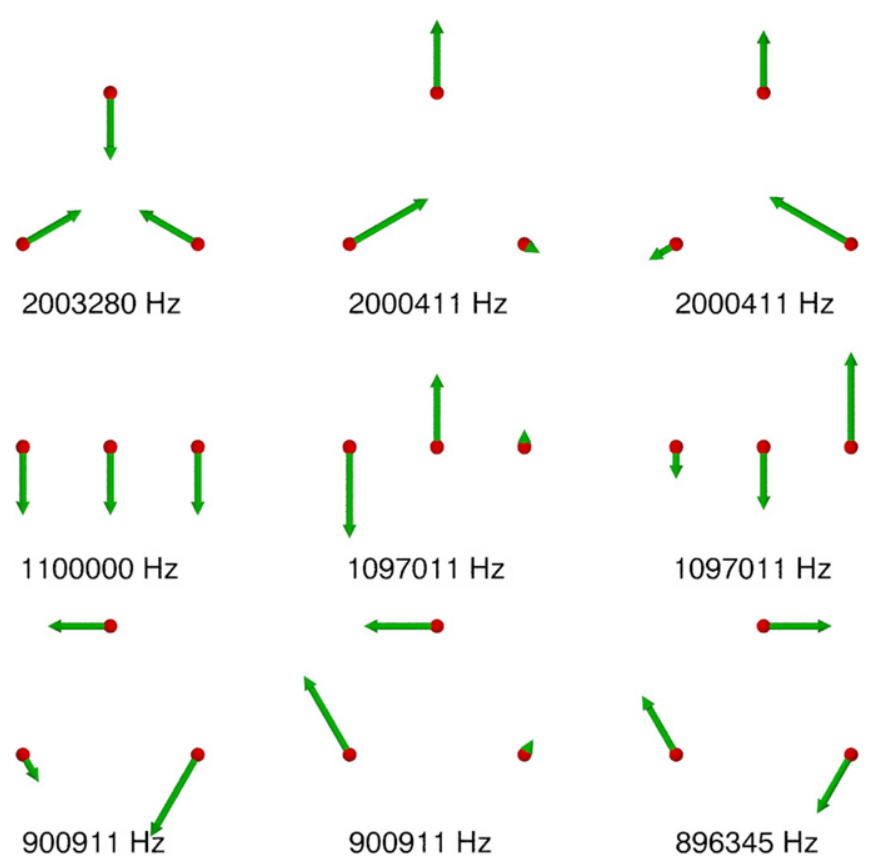

Figure 10. Example for normal modes in a surface-electrode trap geometry similar to the one discussed in section 6. It consists of three trapping zones (red spheres) arranged in a triangle at mutual distances of $40 \mu \mathrm{m}$. Each trapping zone has a (bare) frequency of $2 \pi \times 2 \mathrm{MHz}$ corresponding to the vibration towards the centre of the triangle, $2 \pi \times 1.1 \mathrm{MHz}$ for the out-of-plane vibration and $2 \pi \times 0.9 \mathrm{MHz}$ for the third, perpendicular direction. The eigenvectors (green arrows) of the nine normal modes for an ion of mass $m=25$ amu in each potential minimum are shown (first and last row: top view for in-plane modes; middle row: side view for out-of-plane modes; see appendix A for details of the calculation). The labels denote the frequencies corresponding to the modes. The (coupled) frequencies make up three triplets close to each bare frequency; two frequencies in each triplet are degenerate. For $\hat{k}_{\mathrm{I}}^{(i)}=\hat{k}_{\mathrm{I}}$ pointing in the direction corresponding to the (bare) $2 \pi \times 2 \mathrm{MHz}$ vibration of one of the trapping zones,

$\omega_{\mathrm{I}} \approx 2 \pi \times 1.725 \mathrm{MHz}$, and equal $\Omega_{\mathrm{I}}^{(i)}=\Omega_{\mathrm{I}}$ we can obtain effective spin-spin couplings $J^{(1,2)}=J^{(1,3)}=-J^{(2,3)} \sim 2 \pi \times 1 \mathrm{kHz}$, which would allow for the evolution to a frustrated spin state. We want to note that the out-of-plane modes must be tilted in an implementation to allow for three-dimensional Doppler cooling. This can, for example, be achieved by applying dc voltages to appropriate electrodes (compare section 6). These dc fields could also be used to achieve/raise the degeneracy of the bare frequencies in the individual potential minima and, hence, switch on/off motional couplings between the ions.

lead to a deviation from the ideal model (constant terms are omitted):

$$
\hat{\mathcal{H}}_{\text {Error }}=2 \sum_{i=1}^{N} \sum_{j=1}^{N} \frac{\alpha_{0}}{\alpha_{3}} J^{(i, j)} \hat{\sigma}_{z}^{(i)}+\hat{\mathcal{H}}_{\delta}+\hat{\mathcal{H}}_{\mathrm{E}}^{\prime \prime} .
$$

The first 'bias' term is further discussed in the context of the experimental realization, see [47] and section 5.1. The second term $\hat{\mathcal{H}}_{\delta}$ can be interpreted as an energy offset, which cancels by applying an appropriate redefinition of the energy scale. As mentioned above, the last term leads to only a small or even negligible error for $\left|\Omega_{\mathrm{S}}^{(i)} \eta_{m}^{(i)} \alpha_{l} / \delta_{m}\right| \ll 1$. For a more detailed discussion of the errors in the simulation of quantum spin Hamiltonians we refer the reader to [125]. 
We want to emphasize that $\left[\hat{\mathcal{H}}_{\mathrm{B}}, \hat{\mathcal{H}}_{\mathrm{J}}\right] \neq 0$, such that the time evolution of the total quantum Ising Hamiltonian $\hat{\mathcal{H}}_{\text {QIsing }}$ cannot be simply described by the time evolutions of $\hat{\mathcal{H}}_{\mathrm{B}}$ and $\hat{\mathcal{H}}_{\text {J separately. }}$

\section{Operations interpreted for experimental quantum simulations}

To realize a QS for a quantum spin Hamiltonian, we have to (1) simulate the spin, provide (2) its initialization and (3) the interaction of this 'spin' with a simulated magnetic field, (4) realize an interaction between several spins (spin-spin interaction) and (5) allow for efficient detection of the final spin state. Additional diversity for QS arises by the capability of precise initialization, control and readout of the motional states.

The mathematical derivation and description of the individual operations have been described in section 3. In this section, we explain in a simplified pictorial way the related generic building blocks in terms of an adiabatic QS of a quantum spin Hamiltonian within a linear chain of ions. No specific ion species or trapping concept are required. A well-suited system to illustrate the generic requirements and to investigate the feasibility of QS in ion traps is given by the quantum Ising Hamiltonian (see equation (3.55)). We want to note that the building blocks already suffice to implement a whole family of quantum spin Hamiltonians.

However, the toolbox for QS is substantially larger (see also section 5.2). (1) Phonons do not have to be restricted to mediate interactions in QC and QS: they were also proposed to simulate bosons, for example atoms in the Bose-Hubbard model [22] or charged particles [126]. (2) Topological defects in the zigzag structure of two-dimensional Coulomb crystals (see figure 13) are proposed to simulate solitons [127].

\subsection{Simulating the spin}

The mutual distance between the ions/spins in linear RF traps is typically of the order of several micrometres (see figure 3). Therefore, the direct interaction between their electronic states remains negligible, which is advantageous, because the related interaction strength could hardly be tuned or even switched off. Therefore, the spin-1/2 states are implemented like qubit states (see section 2.2).

\subsection{Simulating the magnetic field}

Implementing an artificial spin allows us to shape artificial fields to implement a precisely controllable interaction and related dynamics between the 'spin' and the 'field'. To simulate an effective magnetic field, the two electronic states $|\downarrow\rangle$ and $|\uparrow\rangle$ are coupled via electro-magnetic radiation (see section 2.3, operation (a)). The related coherent oscillation of the state population between the two levels can be described in terms of Rabi flopping. In the Bloch sphere picture, the tip of the electronic state vector rotates during one flop continuously from state $|\downarrow\rangle$ to $|\uparrow\rangle$ and vice versa. For continuous coupling this can be interpreted as the precession of a spin exposed to a perpendicular magnetic field.

The rotation matrix in equation (3.30) exactly describes this interaction with a single spin (see also sections 3.2 and 3.4). For example, if we start with $|\downarrow\rangle$ and apply a pulsed rotation $\hat{R}(\pi / 2, \pi / 2)$, we will obtain an eigenstate of $\hat{\sigma}_{x}$, which is abbreviated by $|\rightarrow\rangle:=1 / \sqrt{2}(|\downarrow\rangle+|\uparrow\rangle)$. In the Bloch picture, this corresponds to a $90^{\circ}$ rotation of the Bloch vector around the $y$-axis, such that it will point in the direction of the $x$-axis. Continuing with a second identical rotation we just flip the spin to $|\uparrow\rangle$ as if we applied $\hat{R}(\pi, \pi / 2)$ or a $180^{\circ}$ rotation around the $y$-axis, respectively. However, we can replace the second operation by $\hat{R}(\pi / 2,0)$, which corresponds to a rotation around the $x$-axis. As the state $|\rightarrow\rangle$ is an eigenstate of $\sigma_{x}$ it will not be affected.

Stroboscopic rotations have been introduced in section 3.4 to implement single-qubit gates for a QC. Continuous versions of these single-qubit operations can be interpreted in the context of analogue QS as simulated magnetic field (first term of equation (3.55)).

\subsection{Simulating the spin-spin interaction}

Let us first discuss the implementation of a basic spin-spin interaction close to the original proposal in [21]: two ions are confined in a linear RF trap and a standing wave provides statedependent dipole forces. The ions are located at the same phases $\left(\varphi^{(i)}=0\right)$, such that ions in different spin states are pulled/pushed in opposite directions.

If both ions are in the same spin state, they will be pulled in the same direction. Hence, their mutual distance and mutual Coulomb energy, respectively, remain unchanged. However, if the two spins are in different states, one ion will be pulled and the other one pushed. Their mutual distance and as a result their mutual Coulomb energy will change. This is exactly the essence of a spin-spin interaction, where the energy corresponding to a spin state depends on the states of its neighbours. To interpret interactions as ferromagnetic or antiferromagnetic it is advantageous to consider the mutual Coulomb energy in longer chains of spins (see figure 11).

The technical realization in [47] avoids the difficulties arising from standing waves and resonantly enhances the interaction strengths by implementing the spin-spin interactions with stimulated-Raman transitions as in the case of quantum gates [124] (see section 3.4). In a pictorial interpretation, the standing waves are replaced by 'walking' waves and instead of static displacements we obtain driven oscillations of the ions. However, the mathematical description yields exactly the same spin model in an appropriately chosen frame (see section 3.5). The sign of $J$ can additionally be changed by choosing a different sign for the detunings $\delta_{m}$ from the modes (see equation (3.56)).

\subsection{Geometric phase gate versus adiabatic quantum simulations}

It might be helpful to emphasize the differences and similarities of the interactions in QC and analogue QS: to realize a 
(a)
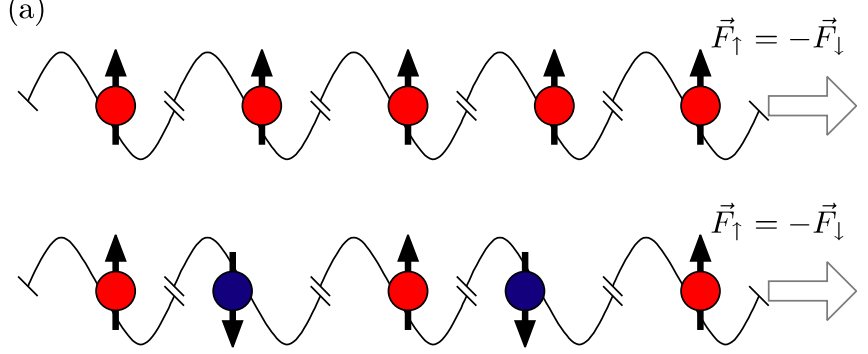

(b)
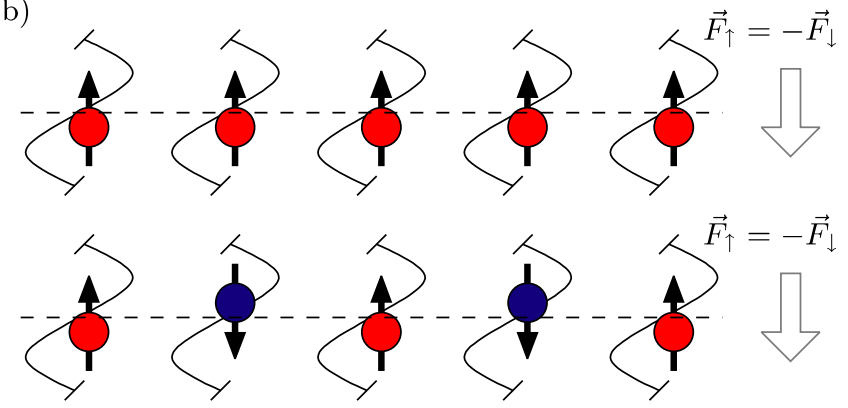

Figure 11. Ion chains superimposed by standing waves providing state-dependent forces in the $(a)$ axial direction and $(b)$ radial direction. All ions are placed at the same phases of the standing waves. (a) If all spins are in the same state, the ions will all be shifted in the same direction without changing their mutual Coulomb energy. However, if every second spin is in the opposite spin state, distances between neighbouring ions will be alternately increased and decreased and the mutual Coulomb energy is increased due to its $1 / d$ dependence. Here $d$ denotes the distance between neighbouring spins. The ferromagnetic order is energetically preferred, such that $J<0$ for this interaction. (b) A chain of ions with the same spin states will again only be displaced and the mutual Coulomb energy will not change. For alternating spin states the distances between neighbouring ions will increase, such that the mutual Coulomb energy will be decreased. (Note that this should not to be confused with the structural zigzag phase-transition (see figure 3), where the radial displacements are typically orders of magnitude larger and spin-independent.) The antiferromagnetic order is energetically preferred, such that $J>0$.

phase gate operation on the radial modes of two qubits, as described in section 3.4, typically one or two isolated modes of motion are selected. The small detuning from the modes is chosen to obtain comparatively large interaction strengths and thus motional excitations. For ions being initialized in the motional ground state the displacements in the respective phase space(s) lead to an average phonon number $\bar{n} \sim 1$ at the point of maximal motional excitation and to a significant entanglement between electronic and motional states. However, this entanglement vanishes at the end of the gate and there will be an (maximal) entanglement between the qubit states only (see section 3.4).

In contrast, we consider an adiabatic evolution according to the quantum Ising Hamiltonian in the case of analogue QS (see section 5.1 for the experimental protocol). We have to make sure that the entanglement between electronic and motional states remains small at any time during the simulation (see discussion of errors in section 3.5). Additionally, running the simulation on many spins simultaneously will result in contributions from many motional modes simultaneously. As a result, a large detuning from all modes has to be chosen, such that the difference of the radial frequencies can be neglected and a net effect from all modes remains. Choosing the right parameters allows the simulation of spinspin interactions of different strength, different signs and even range of interaction [21].

Furthermore, a scan of the duration of the displacement pulses $T_{\mathrm{D}}$ in geometric phase gates leads to a periodic evolution from $|\downarrow \downarrow\rangle$ to $|\uparrow \uparrow\rangle$ and vice versa (see figure 8).

In contrast, the distinct contributions $\left(\hat{\mathcal{H}}_{\mathrm{B}}\right.$ and $\left.\hat{\mathcal{H}}_{\mathrm{J}}\right)$ of the quantum Ising Hamiltonian are not stroboscopically alternated but applied simultaneously. As mentioned in section 3.5, the time evolution according to the quantum Ising Hamiltonian is not simply the time evolution according to $\hat{\mathcal{H}}_{\mathrm{B}}$ and $\hat{\mathcal{H}}_{\mathrm{J}}$ separately. As a consequence, applying the spin-spin interaction for a longer duration and/or increasing its strength does not alter the state anymore.

\section{Towards simulating many-body physics}

In the first part of this section we want to assemble the building blocks described above to illustrate how an analogue QS of a quantum spin Hamiltonian can be implemented. For this purpose, we will describe the realization of first proof-ofprinciple experiments on the quantum Ising Hamiltonian (see equation (3.55)). In the second part we aim to summarize, to the best of our knowledge, the existing proposals addressing many-body physics with the described and available toolbox.

\subsection{Proof-of-principle experiments on quantum spin Hamiltonians}

First, we will describe the basic implementation of the experimental protocol on the axial modes for the case of two spins [47], as illustrated in figure 12. Subsequently, we will emphasize the differences and additional information explored in $[48,49]$. For the details on the individual experimental parameters we refer to these references.

For the case of two spins, the protocol has been realized following five steps. (1) The two ions are initialized by Doppler cooling, sideband cooling and optical pumping (see section 2.4) in the state $|\downarrow \downarrow\rangle\left|n_{\mathrm{STR}}=0\right\rangle$. (2) Both spins are prepared by a common $\hat{R}(\pi / 2, \pi / 2)$ rotation in the $\hat{\sigma}_{x}^{(i)}$ eigenstate $|\rightarrow \rightarrow\rangle\left|n_{\mathrm{STR}}=0\right\rangle$. (3) An effective magnetic field of amplitude $B_{x}$ is applied equivalent to a continuous $\hat{R}\left(2 \Omega_{\mathrm{M}} t, 0\right)$ rotation (see equations (3.55) and (3.56)). At this step, the state $|\rightarrow \rightarrow\rangle\left|n_{\mathrm{STR}}=0\right\rangle$ represents the ground state of the first term of the quantum Ising Hamiltonian in equation (3.55) that can be 'easily' prepared. Note that the rotation is slightly off-resonant to mimic an additional $\hat{\sigma}_{z}$ interaction counteracting the 'bias' field (see also [47]). (4) The effective spin-spin interaction $J$ is ramped up adiabatically with respect to the timescale $1 / \Omega_{\mathrm{M}}$ defined by the simulated magnetic field, until $|J| \gg B_{x}$. The system adiabatically evolves into its new ground state, which is an equal superposition of the two energetically preferred states of the ferromagnetic order: $1 / \sqrt{2}(|\downarrow \downarrow\rangle+|\uparrow \uparrow\rangle)$. (5) Finally, both interactions are switched off. The readout of the final spin state is performed by state-dependent detection. This 


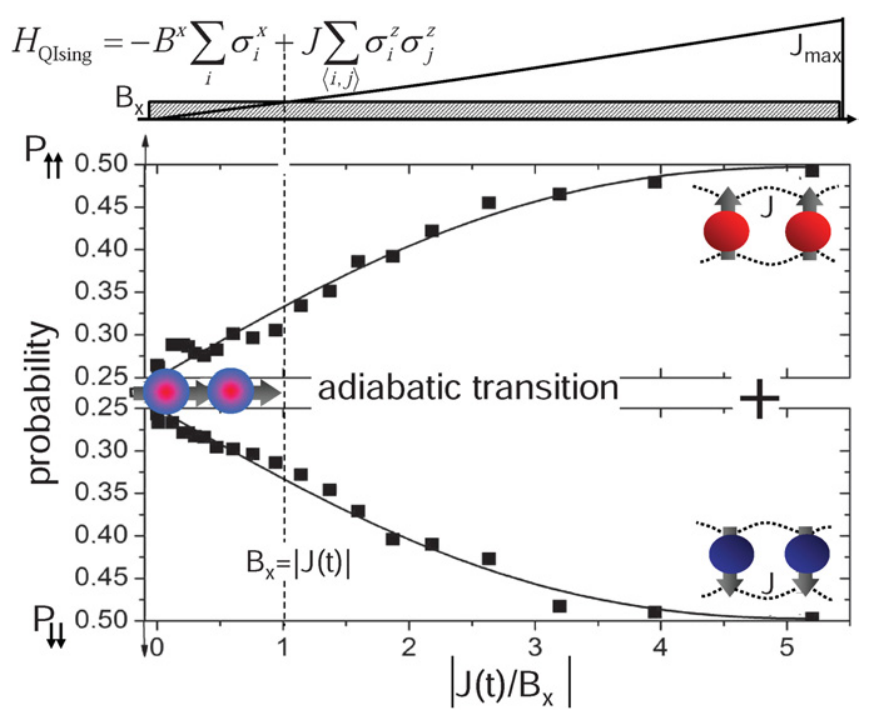

Figure 12. Probability of finding two spins in either of the states $|\downarrow \downarrow\rangle$ or $|\uparrow \uparrow\rangle$ after an adiabatic QS of the quantum Ising Hamiltonian as a function of $\left|J / B_{x}\right|$, starting within paramagnetic order. The experimental protocol (top) consists of the interactions applied simultaneously including an adiabatic increase in $|J|$ to transfer the system from the former ground state $|\rightarrow \rightarrow\rangle$ to the new one (bottom). We achieve a maximal probability of

$P_{\uparrow \uparrow}=P_{\downarrow \downarrow}=(49 \pm 1) \%$ to observe one of the states $|\downarrow \downarrow\rangle$ and $|\uparrow \uparrow\rangle$ corresponding to a ferromagnetic order and define the quantum magnetization to be equal to $P_{\uparrow \uparrow}+P_{\downarrow \downarrow}=(98 \pm 2) \%$. We derive the fidelity for the entangled state $1 / \sqrt{2}(|\downarrow \downarrow\rangle+|\uparrow \uparrow\rangle)$ to approximately $F=0.88$ by a parity measurement (compare [47] and section 3.4).

projects the spin state to one out of the four eigenstates of the measurement basis $(|\downarrow \downarrow\rangle,|\downarrow \uparrow\rangle,|\uparrow \downarrow\rangle,|\uparrow \uparrow\rangle)$. Steps (1)-(5) are repeated many times to obtain the populations related to these states.

To investigate the degree of entanglement of the final spin state, an additional parity measurement is performed as in the case of the geometric phase gates (see section 3.4). The populations of $|\downarrow \downarrow\rangle$ and $|\uparrow \uparrow\rangle$ as a function of $|J| / B_{x}$ and the entanglement fidelity are summarized in figure 12.

The experimentally observed entanglement of the final states confirms that the transition from paramagnetic to ferromagnetic order is not caused by thermal fluctuations that drive thermal phase transitions, but by the so-called quantum fluctuations $[45,128]$ driving QPTs in the thermodynamic limit at zero temperature. In this picture tunnelling processes induced by $B_{x}$ coherently couple the degenerate states $|\uparrow\rangle$ and $|\downarrow\rangle$ with an amplitude $\propto B_{x} /|J|$. For $N$ spins the amplitude for the tunnelling process between $\left|\Psi_{N \uparrow}\right\rangle=|\uparrow \uparrow \ldots \uparrow\rangle$ and $\left|\Psi_{N \downarrow}\right\rangle=|\downarrow \downarrow \ldots \downarrow\rangle$ is proportional to $\left(B_{x} /|J|\right)^{N}$, since all $N$ spins must be flipped. In the thermodynamic limit $(N \rightarrow \infty)$ the system is predicted to undergo a QPT at $|J|=B_{x}$. At values $|J|>B_{x}$ the tunnelling between $\left|\Psi_{\infty \uparrow}\right\rangle$ and $\left|\Psi_{\infty \downarrow}\right\rangle$ is completely suppressed. In our case of a finite system $\left|\Psi_{2 \uparrow}\right\rangle$ and $\left|\Psi_{2 \downarrow}\right\rangle$ remain coupled and the sharp QPT is smoothed into a gradual change from paramagnetic to ferromagnetic order (see figure 12).

It has to be noted that the performance of such a simulation on a large number of spins in a one-dimensional chain requires several technical improvements. Recently, a group at the
University of Maryland pioneered a substantial step for scaling by investigating the emergence of magnetism in the quantum Ising model using up to nine ions [49]. To achieve these results they mediated the interactions via the radial modes of motion [21, 122] (see also section 3.4). Furthermore, they implemented the effective spin-spin interactions in a rotated frame using Mølmer-Sørensen interactions [85,87] on robust hyperfine clock states. Thereby, they do not depend on the phases $\varphi^{(i)}$ of the laser beams at the sites of the ions. To perform an adiabatic transition, the simulated magnetic field has been adiabatically turned off, while the effective spin-spin interaction remains constant.

Their results allow much more than simply increasing the number of spins: they enter a new regime of intriguing questions. The crossover of the quantum magnetization [47] from paramagnetic to ferromagnetic order is sharpening as the number of ions is increased from two to nine, 'prefacing the expected QPT in the thermodynamic limit' [49]. Even though the results can still be calculated on a classical computer, they provide a possibility to critically benchmark QS aiming for only slightly larger systems.

Increasing the number of ions to three and adapting the individual spin-spin interactions including their signs allows spin frustration to be addressed in the smallest possible magnetic network [48]. Spin frustration of the ground state can be pictorially understood in a two-dimensional triangular spin lattice featuring antiferromagnetic spin-spin interactions. Here, it becomes impossible for neighbouring ions to have pairwise opposite states. Classically, two ions will adopt different states, while the state of the third one is undetermined. During an adiabatic evolution of the quantum mechanical system (starting from the paramagnetic order) nature will choose a superposition of all degenerate states, leading to massive entanglement in a spin-frustrated system. In the realization of the experiment, the three ions are still trapped in a one-dimensional chain. However, almost complete control over the amplitudes and signs of $J^{(1,2)}, J^{(2,3)}, J^{(3,1)}$ is gained by coupling to particular collective modes of motion and choosing appropriate detunings [48].

It has to be mentioned that for an increased number of spins the energetic gap between ground and excited states further shrinks and the requirement on adiabaticity enforces longer simulation durations related to a longer exposure to decohering disturbances. Still, as mentioned in section 1, the influence of decoherence might destroy the entanglement within the system, but this might not be relevant for the observable of interest. Here it will be crucial to investigate the role of the decoherence effects with respect to the specific analogue QS.

With respect to digital (stroboscopic) QS it should be emphasized that no quantum error correction is required for proof-of-principle experiments on a few ions. Promising results of a stroboscopic version of the simulation of the quantum Ising Hamiltonian with two spins have been shown recently [129]. 


\subsection{Systems featuring many-body physics proposed for} analogue quantum simulations

Condensed matter met atomic, molecular and optical physics not so long ago, when trapping techniques for ultracold neutral atoms and ions allowed experimentalists to generate lattices and crystals, where models from solid-state physics may be implemented. Combining the fields has led to a very rich interdisciplinary research activity, as well as to several misunderstandings between scientists looking at the same system from different points of view. In the particular case of trapped ion experiments, the outlook for quantum simulation of many-body models is very exciting, but some knowledge on the details of this physical system is required to understand both the limitations and the amazing possibilities of this setup.

In the following we review many-body models that have the potential to be simulated with trapped ions. There have been several contributions both from theory and experiments to this research line. Most of them share the common feature that they are inspired by known models from condensed matter physics, but their implementation with trapped ions turns out to lead to a rich variety of new physical phenomena, which may even require new theoretical paradigms that go beyond the conventional ones in the solid state. The three main reasons for this are (1) trapped ion experiments are naturally performed in a non-equilibrium regime, whereas solid-state physics typically deals with thermal equilibrium, (2) trapped ion systems may in principle be controlled and measured at the single particle level, (3) ion crystals are typically mesoscopic systems, in the sense that they may reach a number of particles (spins, phonons, etc) large enough to show emergent many-body physics, but still finite size effects are important. All those peculiarities have to be kept in mind, since they provide us with unique features for analogue QS.

\subsubsection{Quantum spin models. Following the experimental} advances in QIP, the most natural degree of freedom to be used for QS seems to be the electronic states for spins and the phonons to mediate their mutual interactions. However, one has to identify conditions where interesting phenomena arise, such as, for example, quantum critical phases. This has already lead to the promising proof-of-principle experiments discussed above.

A unique feature that we can exploit with trapped ions is the fact that the effective spin-spin interactions can be implemented showing a dipolar decay, $J^{(i, j)} \propto 1 /|i-j|^{3}$. In the case of the Ising interaction, the cubic dependence does not change the critical universality class of the model, as shown, for example, by the numerical calculations in [125]. However, even in this case, long-range entanglement is induced by the long-range interaction, which is absent in conventional nearest-neighbour quantum Ising chains. On the other hand, when considering other interacting schemes, such as the $X X Z$-Hamiltonian, the dipolar interaction may lead to the formation of quasi-crystalline phases of spin excitations [130]. Furthermore, the simulation of the hexagonal Kitaev model with ions in an optimized, two-dimensional surface-electrode trap has been proposed [131].
Moreover, intrinsic properties of the trapped ion crystal, such as the linear-zigzag transition, have been demonstrated to be a QPT of the universality class of the Ising model in a transverse field [132, 133].

Several pieces have been added to the toolbox of quantum simulation, which definitely allow us to explore physics beyond conventional solid-state paradigms. For example, a theoretical proposal has been presented to implement models, whose ground states show topological features [134]. Also, methods to implement three-body spin-spin interactions have been designed, see [135]. Finally, dissipation in trapped ion systems has been proved to be useful to engineer quantum phases that arise as steady-state of dissipative processes [136]. The many-body physics of dissipative systems is a much more unexplored area than equilibrium properties, even for theorists. For that reason, adding dissipation to quantum magnetism opens an exciting perspective for trapped ions.

5.2.2. Interacting boson models. A variety of exciting quantum many-body systems may also be simulated using the collective motional degrees of freedom (phonons) to realize models of interacting bosons. In particular, whenever the motional coupling between ions is small compared with the trapping frequency, the phonon number is conserved and becomes a good quantum number to characterize the quantum state of the system. This principle was introduced and exploited in [22] to show that the physics of radial modes in Coulomb chains is effectively described by a Bose-Hubbard model. Vibrational couplings between two ions, say 1 and 2 , induced by the Coulomb interaction, have a typical form $\propto \hat{x}^{(1)} \hat{x}^{(2)}$, where $\hat{x}^{(i)}$ is the ion displacement operator. Under the approximation of phonon number conservation, those terms become tunnelling couplings of the form $\left(\hat{a}_{1}^{\dagger} \hat{a}_{2}+\right.$ h.c. $)$. The same idea applies to quartic anharmonicities of the trap, which yield Hubbard interactions, $\left(\hat{a}_{m}^{\dagger} \hat{a}_{m}\right)^{2}$. Anharmonicities may be induced and controlled with optical forces, as shown in [22]. This analogy between phonons and interacting bosons opens an exciting avenue of research, where experiments might be relevant even with a single ion, realizing a single anharmonic quantum oscillator.

The ground state of those phonon-Hubbard models in Coulomb chains was extensively studied in [137], where it was shown that phonon Luttinger liquid phases may arise. Very recent experiments indeed show the tunnelling of phonons between ions trapped by different potentials, realizing thus an important step towards the use of phonons for quantum simulation [138,139]. Exploiting phonon tight-binding models has also been shown to allow the implementation of models with disorder showing Anderson localization [140], as well as synthetic gauge potentials using periodic driving of the trap frequencies, see [126]. Using dipole forces acting on ions confined in a microtrap array (see section 6), motional couplings can be controlled such that phonons simulating charged particles experience synthetic gauge fields.

5.2.3. Spin-boson models. The natural convergence of the proposals presented above leads to the quantum simulation of 
spin-boson models. This is a paradigmatic model for quantum impurities in solids, which typically describes a single spin coupled to a continuous bath of harmonic oscillators with a power-law spectral density. Surprisingly, the coupling of the electronic levels of a single ion to the axial phonons of a Coulomb chain yields a spin-boson model with a quasi-ohmic spectral density [44]. The physics to be simulated here is equivalent to some celebrated models in condensed matter physics, such as the Kondo effect. The finite size effects that are intrinsic of trapped ion systems turn out to yield features beyond the conventional physics of these models, in particular quantum revivals associated with the reflection of vibrational waves along the chain. Quite recently, it has been proposed to study a situation in which spins and phonons are coupled, in such a way that a Jaynes-Cummings-Hubbard model is simulated [141]. In this model phonons follow a tight-binding Hamiltonian and, in addition, they are locally coupled to spins. The system has been shown to undergo a superfluid-Mott insulator QPT.

\subsubsection{Inhomogeneous many-body models: impurities and} topological defects. The tools for QS in ion traps are not restricted to electronic and motional degrees of freedom only. It has been proposed to exploit impurities in the Coulomb crystal. On the one hand, for example, by embedding ion(s) of a different species (different mass) into the crystal and taking advantage of the altered spectrum of the modes and scattering of phonons [142] and the option to include larger simulated spins $(S>1 / 2)$ [143]. On the other hand, by creating localized topological defects within the more dimensional structure of the Coulomb crystal (see figure 13). In [127] it was suggested to induce a structural phase transition from a linear chain of ions (see figure $3(b)$ ) to a zigzag structure (see figure $3(c)$ ), for example, by lowering the radial confinement. Changing the parameters in a non-adiabatic way (fast compared with the phonons mediating information within the crystal) should cause independent domains of 'zigzag' and 'zagzig' structure, respectively. At their clash, topological defects were predicted and have recently been observed (see figure 13). The number of the created defects should scale according to the Kibble-Zurek prediction [144-146]. The defects themselves can be interpreted as solitons [127]. Solitons are defined as localized solutions of nonlinear systems, which depend essentially on nonlinearity. Such solitons have a unique spectrum of frequencies with modes which are localized to the soliton and whose frequency is separated by a gap from the other phonons. A quantum mechanical time evolution of these modes was calculated numerically and it is expected to remain coherent for hundreds of oscillations [147]. QS could allow us to explore their potential applications for QIP [127] as well as the quantum behaviour of these 'objects' themselves. Solitons appear in all branches of the natural sciences and have been extensively investigated in solid-state systems [148]. Among others, classical solitons were observed in waveguide arrays $[149,150]$ and Bose-Einstein condensates (BECs) [151], where they are mean field solutions. Discrete solitons were investigated in the Frenkel-Kontorova (FK) model $[152,153]$, which describes chains of coupled particles interacting with a local nonlinear potential. In a different

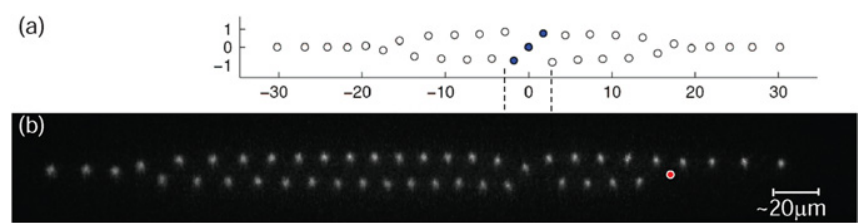

Figure 13. Topological defects in two-dimensional Coulomb crystals (compare figure 3(c) for a comparable crystal without defects). Changing the experimental parameters non-adiabatically during a structural phase transition from a linear chain of ions to a zigzag structure, the order within the crystal breaks up in domains, framed by topologically protected defects that are suited to simulate solitons. (a) Numerical simulations for 33 ions predicting a localized topological defect at the position of the marked (blue) ions. (Courtesy of Benni Reznik and Haggai Landa.) (b) CCD image of 45 laser cooled $\mathrm{Mg}^{+}$ions providing clear evidence of the topological defect indicated by the zigzag-zagzig transition. The crystal contains a non-fluorescing molecular ion $\left(\mathrm{MgH}^{+}\right)$at the red mark. (Courtesy of Günther Leschhorn and Steffen Kahra of the group at MPQ.)

realization, a variant of the FK model can also be realized in the ion trap by adding an optical lattice to a linear chain [154-156].

The important requirement to address any of these intriguing models will be to increase the number of ions and the dimensionality of the system. Trapping ions in twodimensional arrays would allow the study of hard-core boson phases, showing the effect of frustration, quantum spin liquid phases and quantum states with chiral ordering [157]. Two approaches for scaling will be described in more detail in the following sections.

\section{Scaling analogue quantum simulations in arrays of radio-frequency surface-electrode traps}

One possible way to overcome the limitations on scalability of trapped ions in a common potential well (see section 2.1) is to store them in an array of individual RF traps.

\subsection{One-dimensional radio-frequency surface-electrode traps}

Conventional RF traps with their three-dimensional geometry of electrodes (see figure 2) individually fabricated with conventional machining were unique 'masterpieces' with unique characteristics.

In 2005 and 2006, a group at NIST pioneered the miniaturization of RF traps by projecting the electrodes onto a surface $[73,74]$ (see figure 14(a)), very similar to chip traps for neutral atoms [158]. Introducing photolithographic techniques for the trap fabrication opened up exceptional precision and the production of small series of identical traps, see for example $[159,160]$. Within these linear RF surface-electrode traps, motional ground state cooling was achieved at a height of the ion over the electrode surface of $h=40 \mu \mathrm{m}$ and with a comparatively small motional heating rate of the order of 1 quantum $\mathrm{ms}^{-1}$ [74].

Motional heating rates scale with $\sim h^{-4}$ [161]. The exact heating mechanisms are not yet fully understood and the groups 
(a)

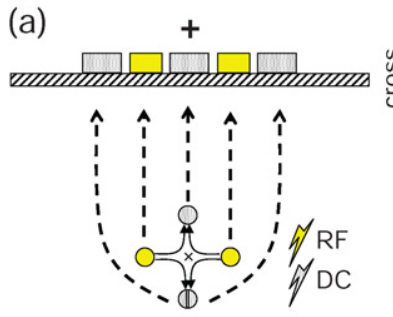

(b)

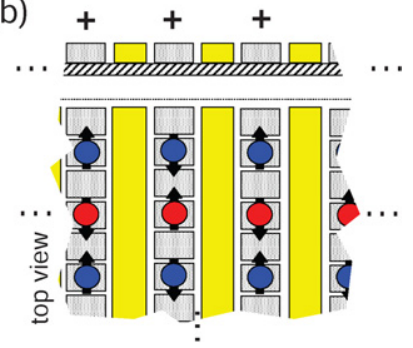

Figure 14. Schematic to illustrate the projection of the electrodes of the RF (yellow) and dc (shaded) electrodes on a surface as a way to scale towards two-dimensional arrays of ions. The black crosses indicate the positions of the minima of the pseudopotentials. (a) Cross section of the electrodes of a conventional linear RF trap with three-dimensional geometry and the electrode structures projected onto a surface. The dashed arrows point at the new location of the electrodes, the white areas represent isolating gaps. (b) Cross section (upper part) and top view (lower part) of the stripe electrodes. It has been proposed to concatenate several linear RF surface-electrode traps as depicted in $(a)$ as a basic unit to span a two-dimensional array of ions [164] (red and blue disks representing ions in opposite spin states). For sufficiently small mutual ion distances and decoherence rates of the ions, this is an approach to scale analogue QS.

at NIST, in Berkeley, at MIT and others are currently putting a lot of effort into further investigations. However, the groups at MIT [162], NIST and the University of Maryland demonstrated a significant reduction of the heating rates in cryogenic (surface electrode) traps for QC purposes (see also [50]). The inverse of these heating rates has long been compared with typical operational durations of a $\mathrm{QC}$ of tens of microseconds (see also section 3.4).

For scaling towards a universal QC it might suffice to interconnect linear ion traps via junctions on a twodimensional surface to a network of one-dimensional traps [163], realizing the 'multiplex ion trap architecture' [75]. That is, ions are proposed to be shuttled between processor and memory traps only interacting in the processor traps. This would allow the subdivision of the large total number of ions into small groups in many individual traps and to reduce the local requirements to a technically manageable effort. One-dimensional RF surface-electrode traps with more than 150 individual dc electrodes and several junctions have been realized [163], allowing ions at moderate heating rates to be shuttled.

In addition, the opportunity arose to deliver identical traps to different groups. One example is the linear RF surface-electrode trap (denoted by 'Sandia Linear Trap' in the following) [159], which was designed by the groups in Oxford, Innsbruck and Sandia National Laboratories. The latter fabricated a small series of identical replicas. The traps have been tested in several laboratories and the individually measured trapping parameters are in good agreement with the design values. Publications are in preparation by the groups at Oxford and Sandia (see also [159]).

It has to be emphasized that pursuing the multiplex approach for scaling universal QC is not applicable to the proposed analogue QS, where the ensemble of spins is supposed to evolve uniformly as a whole.

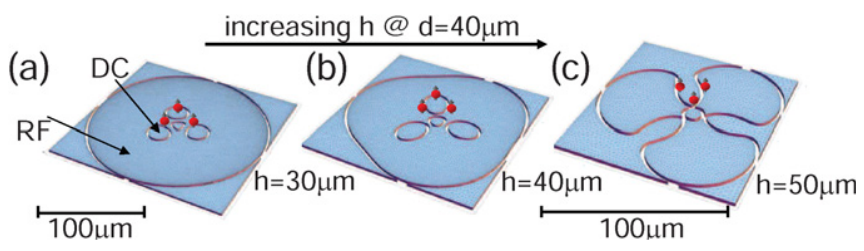

Figure 15. Illustration of the optimization results for the electrode structure for a basic triangular lattice with respect to the height of the ions above the traps at constant mutual ion distance. The white gap isolates RF and dc patches. The three red disks symbolize three ions at a constant distance of $d=40 \mu \mathrm{m}$, hovering above the surface at a height of $(a) h=30 \mu \mathrm{m},(b) h=40 \mu \mathrm{m}$ and $(c)$ $h=50 \mu \mathrm{m}$. (Courtesy of Roman Schmied.)

\subsection{Optimized two-dimensional arrays of radio-frequency surface-electrode traps}

Shortly after the invention of RF surface-electrode traps it was proposed to concatenate linear traps sufficiently close, such that the ions experience mutual Coulomb interaction in two dimensions [164] (see figure 14(b)). However, for a real two-dimensional lattice at sufficiently small and uniform ion distances of $d \leqslant 40 \mu \mathrm{m}$ in two dimensions, this proposal requires the ions to approach the disturbing surface to $h \leqslant$ $d / 2=20 \mu \mathrm{m}$ [73].

Schmied et al implemented a method to calculate the global optimum of the electrode shapes for arbitrary trap locations and curvatures (originally only for periodic boundary conditions) [165]. The gaps between neighbouring electrodes were neglected. The authors exemplarily optimized a trap array with comparatively stiff horizontal confinement.

The idea of optimizing electrode structures can also be used for designing traps for analogue QSs with partially converse requirements. In a collaboration of R. Schmied, NIST, Sandia National Laboratories, and us, such a surfaceelectrode trap has been designed and is currently in fabrication. The trap will provide three trapping zones arranged in a triangle (similar to figure 15) and is intended as a first step towards larger arrays of ions. For this purpose, the optimization method was extended to finite-sized traps.

It has to be emphasized that there are currently several proposals and approaches for arrays of surface-electrode traps mainly for QC. Groups in Berkeley and Innsbruck aim at trap arrays with individually controlled RF electrodes. They have the advantage of selectively lowerable trap frequencies for individual traps and thus increasable interaction strengths between ions in different traps, while especially the height of the ions above the surface can be larger and the trap depth of other traps can be sustained [166] (see also the discussion in the following subsections). This approach can in principle be extended to quasi-micromotion-free shuttling of ions in arrays of RF traps [167] at the expense of precise control of the RF voltage for each RF electrode. Another proposal suggests individual coils to be included for each trap to allow for laser-less interactions mainly for QS [168]. Different trap geometries specifically for QSs have been designed by a group in Sussex [169]. Arrays of Penning traps with surface electrodes are advanced by the groups at Imperial College [170] and the University of Mainz [171]. 
In the following subsections we discuss the optimization goals for a surface-electrode trap for an analogue QS, their implications and the perspectives for scalability of this approach.

6.2.1. Maximization of interaction strengths. The crucial prerequisite for QSs is to maximize the interaction strength (see section 3). As opposed to the QC case with multiple ions in a common potential, this has to be achieved in QS for ions in individual potentials. The increased mutual ion distances in arrays of individual traps substantially reduces the strength of the effective spin-spin interaction. It has be taken into account that the conditional forces have a limited strength, for example, because the laser power is limited or the assumptions in the theoretical model impose constraints as for the quantum Ising Hamiltonian (see section 3.5). However, a reduced stiffness of the individual potentials compared with the example [165] (trap frequencies on the order of $2 \pi \times 20 \mathrm{MHz}$ ) results in larger displacements of the ions by the same forces. This is related to an increased mutual Coulomb energy and thus larger interaction strengths. Still, a lower bound for the trap frequencies (on the order of $2 \pi \times 1 \mathrm{MHz}$ for $\mathrm{Mg}^{+}$) is imposed by the constraints for efficient ground state cooling.

6.2.2. Minimization of decoherence. The ions will inevitably approach the disturbing electrode surfaces, if the distance between the individual traps is reduced. We now reinvest the reduced requirements on the stiffness of the horizontal confinement to increase the height of the ions above the surface $h$ keeping the mutual ion distances $d$ constant. Some results for the scenario of a basic triangular lattice are depicted in figure 15, which demonstrates the adapted shape of the electrodes due to different optimization goals. Note that the influence from electrodes of neighbouring traps increases for an increased height $h$. The optimization allows for an increase in the height by more then a factor of 2 , still maintaining realistic trapping parameters (see below). Hence, the related motional heating rates (in units of energy per time) are expected to be reduced by more than an order of magnitude. In addition, the increased $h$ should help to protect the electrodes from the high intensity of the laser beams parallel to the electrode surfaces.

We additionally include required isolating gaps between electrodes into subsequent simulations to deduce deviations in the resulting trapping potential [172] (see figure 15). The influence of the gaps turned out to be negligible for the example shown in figure 15, however, for further miniaturized traps these influences will grow in importance due to technically limited gap sizes.

6.2.3. Maximization of the lifetime of trapped ions. The reduced frequencies and increased height above the surface come at the price of a reduced trap depth. First, sufficiently deep potentials have to be provided to assure adequate loading rates out of thermal atomic beams, preferably via efficient photoionization $[173,174]$. Second, sufficient lifetimes for many ions within the potentials of scaled traps have to be achieved. Currently, the average lifetime in a room
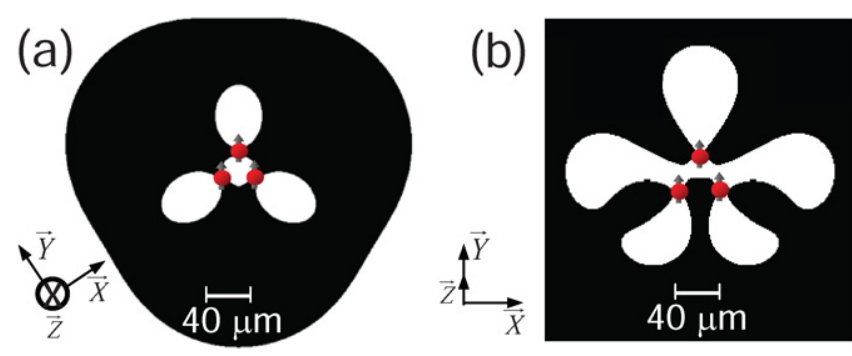

Figure 16. Electrode structures for basic triangular lattices with different orientation and tilt of the principal axes. Red disks symbolize ions trapped in the potential minima for parameters comparable to those in figure $15(b)$. (a) One principal axis points in the vertical direction with the $X$ - and $Y$-axis lying in the horizontal plane of the electrodes. The $X$-principal axis of each trap points towards the centre of the triangle. $(b)$ The respective principal axes of all traps point in the same direction and additionally the $Z$-axis is tilted with respect to the surface by more than $10^{\circ}$, which results in a different symmetry of the electrodes. The tilt of the $Z$-axis is essential to reach all spatial degrees of freedom with laser beams restricted to a plane parallel to the electrodes. (Courtesy of Roman Schmied.)

temperature surface-electrode trap exceeds one hour (for the Sandia Linear Trap operated in our laboratory).

Deeper trapping potentials for surface traps were already achieved by a conductive mesh with controlled voltage $(85 \%$ light transmittance) a few millimetres above the electrode surface [175]. It has also been successfully tested for the Sandia Linear Trap. The mesh shields the ions from charges on the camera viewport and provides a wavelength-independent alternative to a conductive coating (see, for example [176]).

6.2.4. Control of the symmetry of interaction. We additionally gain control over the individual orientations of trap axes or the relative orientations of axes of different traps, respectively (see figure 16). This allows the interaction to be shaped for a given direction of motional excitation between ions in different traps (see section 3). It also allows cooling of all spatial degrees of freedom with laser beams, which have to propagate parallel to the trap surface to minimize scattering off the surface. We can rotate the individual trap axes from pointing towards the centre of the structure (see figure 16(a)) into a parallel alignment and additionally include the required tilt of the vertical $(Z)$ axes, which will result in a different symmetry of the electrodes (see figure $16(b)$ ).

6.2.5. Control of the potential in individual traps. First, splitting dc electrodes into several separately controllable segments allows for the individual compensation of displacements of the ions from the minima of the pseudopotential due to stray fields and space charge effects (compare section 2.1 and see [176,177] for schemes of micromotion compensation). Second, for further scaling, these electrodes can be used to compensate boundary effects. Due to the larger number of inner ions, outer ones would be shifted to larger mutual distances. The further increased density of electrodes on the surface requires their connections in a multilayer structure with vertical wiring (vias) $[159,160]$. 
6.2.6. Estimation of parameters. We estimate the strength of simulated spin-spin interactions for the case of $\mathrm{Mg}^{+}$ions in such devices with currently available laser equipment. We assume a typical laser power of $400 \mathrm{~mW}$ (max. $600 \mathrm{~mW}$ are available) at $280 \mathrm{~nm}$ from an all solid-state laser source [178]. We further assume the beam to have a cylindrical profile with waists of $10 \mu \mathrm{m} \times 100 \mu \mathrm{m}$ and an electrode structure as depicted in figure $16(b)(d=h=40 \mu \mathrm{m})$. For a trap depth of $100 \mathrm{meV}$ and a minimal oscillation frequency of the ions of $2 \pi \times 2 \mathrm{MHz}$, the interaction strengths by far exceed $2 \pi \hbar \times 1 \mathrm{kHz}$.

In a different approach, we could think of using the motional degrees of freedom for QSs. This scheme would have the advantage that bare motional couplings are already in the $2 \pi \times 5 \mathrm{kHz}$ regime. In that sense, they are stronger than effective spin-spin interactions, since the latter are slowed down with respect to the original motional couplings by the requirement of adiabaticity. A recent theoretical proposal by some of us has shown that using periodic modulations of the trapping frequencies, some phenomena from solid-state physics may be simulated, such as photon assisted tunnelling [126] (see section 5.2).

\subsection{Perspectives of our approach}

As depicted in figure $16(b)$, in a first step three ions will reside on the vertices of a triangle and the interaction between the spins can be simulated as in $[47,48]$ (compare section 3.5) or [126] (compare section 5.2). The above parameter estimates should already suffice for proof-of-principle experiments and mesoscopically scaled QS. Motional modes in twodimensional trap arrays will behave similarly to radial modes in linear RF traps for all three dimensions [21,124] and the effective spin-spin interaction will prefer antiferromagnetic order for far, red detuning from all modes. Thus, the systems should give us the possibility to study spin frustrations in a spatial, triangular configuration (see also section 5.1).

Based on the results of these investigations further scaling of the surface-trap architecture to large-scale (triangular) lattices of tens or even hundreds of spins might be pursued (see figure 17). In addition to the optimization of the trapping parameters, further technical difficulties have to be considered.

Decoherence due to motional heating as a result of the vicinity to the electrode surfaces could be mitigated within a cryogenic setup [50,162]. The reduced vacuum pressure could additionally help to increase the lifetime of $\mathrm{Mg}^{+}$, which is currently limited by photochemical reactions with hydrogen (mostly $\mathrm{H}_{2}+\mathrm{Mg}^{+*} \rightarrow \mathrm{MgH}^{+}+\mathrm{H}^{*}$ ) and collisions with heavy components of the rest gas. The reaction can also be inverted by pulsed laser beams [179]. However, scaling the system to tens or hundreds of ions will still require frequent and efficient reloading. Increasing the loading efficiency and preserving the vacuum conditions could be achieved by photoionizing cold atoms from a magneto-optical trap (MOT) [180].

Currently, the available laser power should not impose any restrictions on the realization of systems of few tens of ions (see [178] currently providing up to $600 \mathrm{~mW}$ ). Higher laser powers for magnesium are in reach [181-183] and could
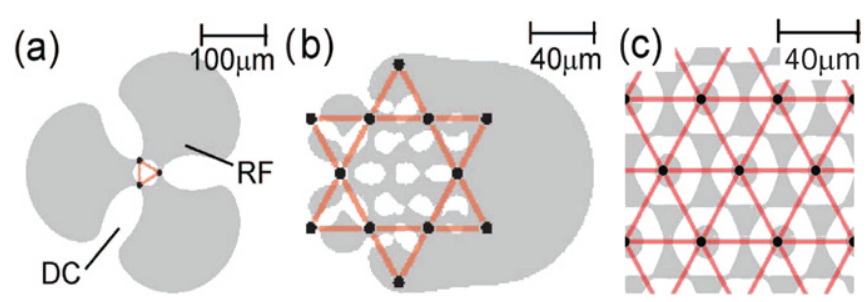

Figure 17. Electrode structures for RF surface-electrode traps scaled for analogue QSs. Black dots symbolize the RF minima, red lines serve as a guide to the eye to emphasize the lattice structures for $(a)$ three, $(b) 12$ and $(c)$ an infinite number of ions/spins. The identical orientation of the principal axes of each RF minimum and non-vanishing components parallel to the trap surfaces (see figure 16) are considered for the latter two. (Courtesy of Roman Schmied.)

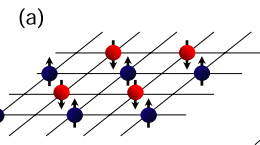

(b)
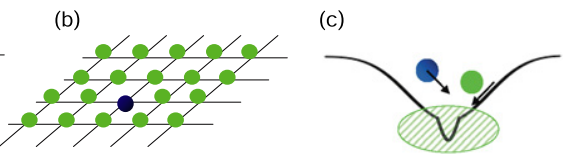

Figure 18. Illustration of new options for analogue QS based on ions (red and blue) and atoms (green) in optical potentials (black lines as a guide to the eye). (a) Ions populate an optical lattice on well separated sites. The Coulomb force still provides a large strength of dipolar (long-ranging) interaction allowing for analogue QS on many-body effects, similar to the proposed approach in arrays of RF surface-electrode traps (see section 6). (b) An ion and atoms populate a common optical lattice and, for example, share the charge via tunnelling electrons. (c) An ion could be cooled sympathetically by cold atoms (for example, a BEC indicated by the green ellipse) $[192,199]$. Since the micromotion of the ion and the related differential motion between atoms and ion becomes negligible in the common optical trap [60], deep equilibrium temperatures are predicted to be achievable, down to a regime where ultra-cold chemistry might dominate the collisions.

allow for even larger arrays of simultaneously coupled ions. In addition to that, efforts in optics, for example arrays of lenses $[184,185]$, fibres integrated into the trap [186] or integrated mirrors [187-189], could provide individual addressing and high light intensities at the position of the ions. To further mitigate the problem of scattered light from surfaces, one could think of realizing surface traps on partially transparent substrates [74]. Alternatively, laser-less coupling could be used as mentioned in section 2.3 [88, 89, 91-93, 168].

Last but not least, it still has to be identified how to measure observables that permit the verification of frustration effects without the need for full (exponentially complex) state tomography.

\section{Scaling quantum simulations based on ions in optical lattices}

Some groups aim to merge the two fields of QS based on ions in RF traps and atoms confined in optical lattices. It has already been proposed to combine Coulomb crystals in a harmonic confinement of a common RF trap of three-dimensional geometry with (commensurate) optical lattices to shape anharmonic trapping potentials providing new possibilities to simulate interactions [157]. Another proposal deals with the 
simulation of the Frenkel-Kontorova model using a standing wave aligned with the trap axis [156]. Kollath et al suggested exploiting a trapped ion to coherently couple (such as a scanning microscope) to the atoms confined in an optical lattice [190].

Optical ion trapping was realized with a single $\mathrm{Mg}^{+}$ion trapped in a dipole trap [80]. We can now dream of spanning an array of ions (even simultaneously with neutral atoms) within an optical lattice. It has to be emphasized that the smaller trap depth of optical traps (see figure 1) renders it highly unlikely that optically trapping charged atoms will allow us to outperform the achievable trapping parameters or coherence times of both, ions in RF traps and of optically trapped neutral atoms. However, in our opinion, this is not required. The advantage of equally and closely spaced traps might be combined with individual addressability and, most importantly, the long-range interaction provided by Coulomb forces between the ions.

In the following, we will first describe how trapping of an ion in a dipole trap was achieved. Still facing a huge variety of challenges, the new possibilities will be discussed afterwards.

\subsection{Trapping of an ion in a dipole trap}

The procedure used in [80] to load a magnesium ion $\left({ }^{24} \mathrm{Mg}^{+}\right)$ into a dipole trap consists of the following steps: an atom is photoionized out of a thermal beam and trapped and Doppler cooled in a conventional RF trap. Next, stray electric fields are minimized at the site of the ion using the ion as a sensor. Then a Gaussian laser beam providing the dipole trap is focused onto the ion and the RF drive of the RF trap is switched off. From that time on, the ion is confined in the dipole trap in the directions perpendicular to the beam propagation. The depth of the dipole trap potential amounts to $U_{0} \approx 2 \pi \hbar \times 800 \mathrm{MHz}$ or $U_{0} \approx k_{\mathrm{B}} \times 38 \mathrm{mK}$, respectively, and the detuning of the dipole trap beam from the relevant transition $\left(\mathrm{S}_{1 / 2} \leftrightarrow \mathrm{P}_{3 / 2}\right)$ to $\Delta \approx-6600 \Gamma$, where $1 / \Gamma$ determines the lifetime of the $\left|\mathrm{P}_{3 / 2}\right\rangle$ state. Static electric fields provide the confinement in the direction of beam propagation. After a few milliseconds the RF drive is switched on again and the presence of the ion can be verified via its fluorescence during Doppler cooling.

For the given parameters a half-life of approximately $2.5 \mathrm{~ms}$ is achieved. This value is in very good agreement with the theoretical predictions, assuming exclusively the heating process related to off-resonant scattering of the trapping light by the ion, the so-called recoil heating. It can be concluded that the heating and subsequent loss of ions from the optical potential is not dominated by heating effects related to the charge of the ion, for example, due to the vicinity of electrodes or fluctuating stray electric fields. Thus, state-ofthe-art techniques for neutral atoms should allow effective enhancement of the lifetime and coherence times [191].

\subsection{Lifetime and coherence times of optically trapped ions}

We aim to increase the lifetime by cooling the ion in the dipole trap. Due to the large ac Stark shift and its large position dependence, simple Doppler cooling within the existing setup is challenging. Possibilities of cooling the ions directly towards the ground state of motion within the dipole potential are currently being investigated theoretically and experimentally.

An alternative approach suggests to use cold atoms or even a BEC to sympathetically cool ions [192]. On longer timescales the approach of cavity assisted cooling of ions in conventional RF traps reported in [193] might also provide long lifetimes without affecting the electronic state of the ion.

Currently, the coherence time of the electronic state of the ion is limited to a few microseconds due to the high spontaneous emission rate. If longer coherence times are required (which is not necessarily the case for every scenario), they can be achieved in two ways. (1) As for two-photon stimulated-Raman transitions the spontaneous emission rate can be reduced by increasing the detuning. A larger beam intensity could sustain the potential depth. (2) Another option would be to work with blue detuned light, where the potential depths can remain identical, however, the ions seek low intensity and exhibit less spontaneous emission.

\subsection{Towards ions and atoms in a common optical lattice}

It has still to be demonstrated that one or several ions can be confined within one- or more-dimensional optical lattices. With currently available laser sources a mutual ion distance within each dimension of the order of tens of micrometres could be achieved, which corresponds to one ion at approximately every 40th to 50th lattice site (see methods in [80]). Therefore the mutual ion distance could be smaller than the currently envisioned distances between neighbouring traps in the RF surface-electrode trap approach (see section 6).

Since the photoionization scheme applied so far ionizes out of a thermal beam of magnesium atoms, the average kinetic energy of the atoms is much larger than the depth of the optical potential and, in addition, the local vacuum is severely affected. The loading efficiency for RF traps could be largely enhanced by ionizing $\mathrm{Mg}$ atoms from a MOT [194], which would also allow direct loading of atoms into an optical trap. In addition, after loading neutral atoms into the lattice, some of them could be photoionized on site.

Ions and atoms confined in a common optical lattice could offer an approach to exploit the physics of charge transfer reactions. This might allow for a complete new class of QS, for example, of solid-state systems, where atoms in a completely occupied lattice (at an initially small density of ions) share electrons by tunnelling causing highly entangled states of the compound system. The resulting quantum manybody dynamics should be governed by the interplay of the quantum state of the trapped neutral atoms and the electron tunnelling from neutrals to ions.

\section{Conclusions}

In the last few years the basic building blocks for a scalable architecture of a quantum information processor (QC) with trapped ions have been demonstrated for a few qubits. Additionally, a large variety of new techniques have already been tested that might considerably extend the available 
toolbox. For example, interactions based on magnetic field gradients and RF fields, fibre-coupled optical support on chip or economically and technologically facilitated cryogenic environments. Despite the fact that it will be a non-trivial challenge to scale the system to approximately $10^{5}$ qubits, no fundamental limitations have been identified so far.

On a shorter timescale, intriguing problems might be studied by realizing analogue quantum simulators (QS), by far exceeding the capabilities of classical computers. They can be based on similar techniques as a potential QC, but with less severe constraints on the fidelity of operations and the number of required ions.

Currently available operational fidelities are predicted to allow for studying many-body physics, for example in systems described by quantum spin Hamiltonians, the BoseHubbard and the spin-boson models. First proof-of-principle experiments simulating Ising type interactions with a few ions have already been successfully demonstrated.

The required increase of the number of ions and the accessible dimensions is proposed within two-dimensional arrays of RF surface-electrode traps. However, the approach is still at the level of proof-of-principle experiments and further challenges might arise during its development. Alternative approaches include Penning traps or optical lattices.

Even though the enthusiasm within this quickly growing field seems to be justified, it has to be emphasized that efficient analogue QS still require more than simply scaling. Examples of other important challenges are (1) to investigate carefully the influence of different sources of decoherence on the fidelity of the simulation. Thus, it must be distinguished for the dedicated application, which decoherence the simulation will be robust against, which decoherence can be considered in the simulation and which decoherence is even essential to be included. (2) To identify possibilities to cross-check the validity of the output or to benchmark it against other QS approaches, as soon as the achieved output is not accessible with a classical computer anymore.

In the future it might be beneficial to combine advantages of several systems for hybrid QS. On longer timescales, the experiences gained by developing an analogue QS based on trapped ions might culminate in approaches incorporating solid-state devices that might allow for 'easier' scaling. With the realization of a universal QC, universal QS will also become accessible.

\section{Acknowledgments}

CS and TS acknowledge support by the Max-Planck-Institut für Quantenoptik (MPQ), Max-Planck-Gesellschaft (MPG), Deutsche Forschungsgemeinschaft (DFG) (SCHA 973/1-6), the European Commission (The Physics of Ion Coulomb Crystals: FP7 2007-2013. grant no. 249958) and the DFG Cluster of Excellence 'Munich Centre for Advanced Photonics'. DP acknowledges support from C.A.M. Project QUITEMAD, RyC Contract Y200200074, and MICINN FIS2009-10061. Martin Enderlein, Thomas Huber, and Hector Schmitz have participated in the measurements of the geometric phase gate utilizing the radial modes of motion presented in section 3.4. Günther Leschhorn and Steffen Kahra have observed and taken the picture of the structural defect in a Coulomb crystal of $\mathrm{Mg}^{+}$ions (see figure 13(b)). Benni Reznik and Haggai Landa provided the corresponding simulation (see figure 13(a)). Roman Schmied kindly provided several simulation results and illustrations of optimized electrode structures of surface-electrode traps (see section 6). We want to thank Geza Giedke and Alex Retzker for discussions and Martin Enderlein, Thomas Huber, and Dietrich Leibfried for comments on the manuscript. We also thank Ignacio Cirac and Gerhard Rempe for their intellectual and financial support.

\section{Appendix A. Normal modes and frequencies}

This section describes a more general derivation of the normal modes and frequencies compared with the one-dimensional treatment for the linear Paul trap as in [195]. The equations are extended to three dimensions and an arbitrary trap potential, as long as the potential at the equilibrium position $\vec{x}_{0}^{(i)}$ of each ion can be well approximated by a harmonic potential.

The position of the $i$ th ion is expressed in the Cartesian coordinates of the laboratory frame

$$
\vec{r}^{(i)}=r_{i} \vec{e}_{X}+r_{i+N} \vec{e}_{Y}+r_{i+2 N} \vec{e}_{Z}
$$

The decomposition into the equilibrium position $\vec{x}_{0}^{(i)}$ and displacements $\vec{x}^{(i)}$ yields

$$
\begin{aligned}
\vec{r}^{(i)} & =\vec{x}_{0}^{(i)}+\vec{x}^{(i)} \\
& =\left(x_{0, i}+x_{i}\right) \vec{e}_{X}+\left(x_{0, i+N}+x_{i+N}\right) \vec{e}_{Y}+\left(x_{0, i+2 N}+x_{i+2 N}\right) \vec{e}_{Z}
\end{aligned}
$$

The Lagrangian for $N$ ions takes the form

$$
\mathcal{L}=\frac{1}{2} M[\sum_{k=1}^{3 N} \dot{x}_{k}^{2}-\sum_{k=1}^{3 N} \sum_{l=1}^{3 N} \underbrace{\frac{1}{M}\left(\frac{\partial^{2} V}{\partial r_{k} \partial r_{l}}\right)_{x_{k}=x_{l}=0}}_{=: a_{k l}} x_{k} x_{l}],
$$

where $M$ denotes the mass of an ion, the index of the partial derivatives signifies its evaluation at the equilibrium positions and $V$ denotes the potential consisting of the trap potential $V_{0}$ and the Coulomb potentials of the ions:

$$
V=V_{0}+\frac{Q^{2}}{8 \pi \epsilon_{0}} \sum_{i=1}^{N} \sum_{\substack{j=1 \\ j \neq i}}^{N} \frac{1}{\left|\vec{r}^{(i)}-\vec{r}^{(j)}\right|} .
$$

Here, $Q$ denotes the charge and $\epsilon_{0}$ the electric constant.

For practical purposes the trap potential can be expressed by the harmonic terms corresponding to each ion:

$$
V_{0}=\frac{1}{2} M \sum_{i=1}^{N} \sum_{j=1}^{3} \omega_{j}^{(i) 2}\left(\vec{r}^{(i)}-\vec{p}^{(i)}\right)^{\mathrm{T}} \vec{d}_{j}^{(i)} \otimes \vec{d}_{j}^{(i)}\left(\vec{r}^{(i)}-\vec{p}^{(i)}\right) .
$$

Here, $\omega_{j}^{(i)}$ denotes the $j$ th frequency of the harmonically approximated potential of the $i$ th ion, $\vec{d}_{j}^{(i)}$ the unity vector of the principle axis corresponding to $\omega_{j}^{(i)}$ and $\vec{p}^{(i)}$ the position of 
the local minimum of the potential for the $i$ th ion. Note that the frequencies, the vectors of the principle axes, and the minima of the potentials become equal for all ions in the special case of a linear Paul trap.

The eigenvalues of the Hessian $A:=\left(a_{k l}\right)$ (see equation (A.4)) yield the squares of the frequencies $\omega_{m}$ of the normal modes and its eigenvectors $\vec{b}_{m}$ determine the ions' motion of the $m$ th mode:

$$
q_{m}=\vec{b}_{m} \cdot \vec{x} \quad \text { with } \quad \vec{x}:=\left(x_{1}, \ldots, x_{3 N}\right) .
$$

With the abbreviations $\vec{q}:=\left(q_{1}, \ldots, q_{3 N}\right)$ and $B:=$ $\left(\vec{b}_{1}, \ldots, \vec{b}_{3 N}\right)^{\mathrm{T}}$, where the $\vec{b}_{m}$ shall be understood as rows of $B$, we can express the relation in a more compact way:

$$
\vec{q}=B \vec{x} \quad \Leftrightarrow \quad \vec{x}=B^{\mathrm{T}} \vec{q} .
$$

Typically, the eigenvalues and eigenvectors of $A$ have to be determined numerically.

\section{Appendix B. Transformations of Pauli operators}

The definitions of the Pauli operators are repeated here to avoid confusions concerning their normalization:

$\hat{\sigma}_{x}:=\left(\begin{array}{ll}0 & 1 \\ 1 & 0\end{array}\right), \quad \hat{\sigma}_{y}:=\left(\begin{array}{cc}0 & -\mathrm{i} \\ \mathrm{i} & 0\end{array}\right), \quad \hat{\sigma}_{z}:=\left(\begin{array}{cc}1 & 0 \\ 0 & -1\end{array}\right)$.

The Pauli operators obey the relations

$$
\begin{gathered}
{\left[\hat{\sigma}_{i}, \hat{\sigma}_{j}\right]=2 \mathrm{i} \epsilon_{i j k} \hat{\sigma}_{k},} \\
\left\{\hat{\sigma}_{i}, \hat{\sigma}_{j}\right\}=2 \delta_{i j}, \\
\hat{\sigma}_{i}^{2}=\hat{\mathbb{1}} .
\end{gathered}
$$

A more convenient notation in some contexts is

$$
\begin{gathered}
\hat{\sigma}_{+}:=\hat{\sigma}_{x}+\mathrm{i} \hat{\sigma}_{y}=\left(\begin{array}{ll}
0 & 2 \\
0 & 0
\end{array}\right), \\
\hat{\sigma}_{-}=\hat{\sigma}_{x}-\mathrm{i} \hat{\sigma}_{y}=\left(\begin{array}{ll}
0 & 0 \\
2 & 0
\end{array}\right)
\end{gathered}
$$

with the normalization as in [50]. They fulfil the following relations:

$$
\begin{aligned}
& {\left[\hat{\sigma}_{ \pm}, \hat{\sigma}_{\mp}\right]= \pm 4 \hat{\sigma}_{z},} \\
& {\left[\hat{\sigma}_{z}, \hat{\sigma}_{ \pm}\right]= \pm 2 \hat{\sigma}_{ \pm} .}
\end{aligned}
$$

The transformations of the Pauli operators into the interaction picture involve terms of the form

$$
\hat{\sigma}_{i}^{\prime}:=\mathrm{e}^{\mathrm{i} \kappa \hat{\sigma}_{z}} \hat{\sigma}_{i} \mathrm{e}^{-\mathrm{i} \kappa \hat{\sigma}_{z}} .
$$

The transformation leaves $\hat{\mathbb{1}}$ and $\hat{\sigma}_{z}$ unchanged. The non-trivial cases $i=x$ and $i=y$ can be calculated using the BakerCampbell-Hausdorff formula

$$
\begin{aligned}
\mathrm{e}^{-\hat{B}} \hat{A} \mathrm{e}^{\hat{B}} & =\sum_{n} \frac{1}{n !}[\hat{A}, \hat{B}]^{\{n\}} \\
& =\hat{A}+[\hat{A}, \hat{B}]+\frac{1}{2}[[\hat{A}, \hat{B}], \hat{B}]+\cdots
\end{aligned}
$$

with $\hat{B}=-\mathrm{i} \kappa \hat{\sigma}_{z}, \hat{A}=\hat{\sigma}_{x / y}=\frac{\hat{\sigma}_{+} \pm \hat{\sigma}_{-}}{m_{+}}, m_{+}:=2$ and $m_{-}:=2 \mathrm{i}$.

The commutators are given by

$\left[\hat{\sigma}_{+} \pm \hat{\sigma}_{-},-\mathrm{i} \kappa \hat{\sigma}_{z}\right]=\mathrm{i} 2 \kappa\left(\hat{\sigma}_{+} \mp \hat{\sigma}_{-}\right)$

$\left[\hat{\sigma}_{+} \pm \hat{\sigma}_{-},-\mathrm{i} \kappa \hat{\sigma}_{z}\right]^{\{2\}}=\mathrm{i} 2 \kappa\left[\left(\hat{\sigma}_{+} \mp \hat{\sigma}_{-}\right),-\mathrm{i} \kappa \hat{\sigma}_{z}\right]$

$=(\mathrm{i} 2 \kappa)^{2}\left(\hat{\sigma}_{+} \pm \hat{\sigma}_{-}\right)$,

$\left[\hat{\sigma}_{+} \pm \hat{\sigma}_{-},-\mathrm{i} \kappa \hat{\sigma}_{z}\right]^{\{2 n-1\}}=(\mathrm{i} 2 \kappa)^{2 n-1}\left(\hat{\sigma}_{+} \mp \hat{\sigma}_{-}\right)$,

$\left[\hat{\sigma}_{+} \pm \hat{\sigma}_{-},-\mathrm{i} \kappa \hat{\sigma}_{z}\right]^{\{2 n\}}=(\mathrm{i} 2 \kappa)^{2 n}\left(\hat{\sigma}_{+} \pm \hat{\sigma}_{-}\right)$.

Hence, the Pauli operators in the interaction picture read

$\mathrm{e}^{\mathrm{i} \kappa \hat{\sigma}_{z}} \hat{\sigma}_{x / y} \mathrm{e}^{-\mathrm{i} \kappa \hat{\sigma}_{z}}=\mathrm{e}^{\mathrm{i} \kappa \hat{\sigma}_{z}} \frac{\hat{\sigma}_{+} \pm \hat{\sigma}_{-}}{m_{ \pm}} \mathrm{e}^{-\mathrm{i} \kappa \hat{\sigma}_{z}}$

$$
\begin{aligned}
= & \frac{1}{m_{ \pm}} \sum_{n} \frac{(\mathrm{i} 2 \kappa)^{2 n+1}}{(2 n+1) !}\left(\hat{\sigma}_{+} \mp \hat{\sigma}_{-}\right) \\
& +\frac{1}{m_{ \pm}} \sum_{n} \frac{(\mathrm{i} 2 \kappa)^{2 n}}{(2 n) !}\left(\hat{\sigma}_{+} \pm \hat{\sigma}_{-}\right) \\
= & \frac{1}{m_{ \pm}} \sum_{n} \frac{(\mathrm{i} 2 \kappa)^{n}}{n !} \hat{\sigma}_{+} \\
& \pm \frac{1}{m_{ \pm}} \sum_{n} \frac{(-\mathrm{i} 2 \kappa)^{n}}{n !} \hat{\sigma}_{-} \\
= & \frac{1}{m_{ \pm}}\left(\mathrm{e}^{\mathrm{i} 2 \kappa} \hat{\sigma}_{+} \pm \mathrm{e}^{-\mathrm{i} 2 \kappa} \hat{\sigma}_{-}\right) .
\end{aligned}
$$

The operators $\hat{\sigma}_{+}$and $\hat{\sigma}_{-}$transform:

$$
\begin{gathered}
\mathrm{e}^{\mathrm{i} \kappa \hat{\sigma}_{z}} \hat{\sigma}_{+} \mathrm{e}^{-\mathrm{i} \kappa \hat{\sigma}_{z}}=\mathrm{e}^{\mathrm{i} 2 \kappa} \hat{\sigma}_{+}, \\
\mathrm{e}^{\mathrm{i} \kappa \hat{\sigma}_{z}} \hat{\sigma}_{-} \mathrm{e}^{-\mathrm{i} \kappa \hat{\sigma}_{z}}=\mathrm{e}^{-\mathrm{i} 2 \kappa} \hat{\sigma}_{-} .
\end{gathered}
$$

\section{Appendix C. Transformations of motional operators}

The creation operator $\hat{a}$ and the annihilation operator $\hat{a}^{\dagger}$ fulfil the relations

$$
\begin{gathered}
\hat{a}|n\rangle=\sqrt{n}|n-1\rangle, \\
\hat{a}^{\dagger}|n\rangle=\sqrt{n+1}|n+1\rangle, \\
{\left[\hat{a}, \hat{a}^{\dagger}\right]=\hat{\mathbb{1}} .}
\end{gathered}
$$

The transformation of the Hamiltonians into the interaction picture requires the knowledge of the transformation of $\mathrm{e}^{\mathrm{i} \xi\left(\hat{a}+\hat{a}^{\dagger}\right)}$

It can be performed using the special case of the BakerCampbell-Hausdorff formula from appendix B again. The commutators appearing in the formula are

$$
\begin{aligned}
{\left[\hat{a}+\hat{a}^{\dagger},-\mathrm{i} \lambda \hat{a}^{\dagger} \hat{a}\right] } & =-\mathrm{i} \lambda\left(\hat{a}-\hat{a}^{\dagger}\right), \\
{\left[\hat{a}+\hat{a}^{\dagger},-\mathrm{i} \lambda \hat{a}^{\dagger} \hat{a}\right]^{\{2\}} } & =-\mathrm{i} \lambda\left[\hat{a}-\hat{a}^{\dagger},-\mathrm{i} \lambda \hat{a}^{\dagger} \hat{a}\right] \\
& =(-\mathrm{i} \lambda)^{2}\left(\hat{a}+\hat{a}^{\dagger}\right), \\
{\left[\hat{a}+\hat{a}^{\dagger},-\mathrm{i} \lambda \hat{a}^{\dagger} \hat{a}\right]^{\{n\}} } & =(-\mathrm{i} \lambda)^{n}\left(\hat{a}+(-1)^{n} \hat{a}^{\dagger}\right) .
\end{aligned}
$$

Hence, the full transformation reads

$$
\begin{aligned}
\mathrm{e}^{\mathrm{i} \lambda \hat{a}^{\dagger} \hat{a}}\left(\hat{a}+\hat{a}^{\dagger}\right) \mathrm{e}^{-\mathrm{i} \lambda \hat{a}^{\dagger} \hat{a}} & =\sum_{n} \frac{(-\mathrm{i} \lambda)^{n}}{n !}\left(\hat{a}+(-1)^{n} \hat{a}^{\dagger}\right) \\
& =\hat{a} \mathrm{e}^{-\mathrm{i} \lambda}+\hat{a}^{\dagger} \mathrm{e}^{\mathrm{i} \lambda} .
\end{aligned}
$$


From this relation we can immediately derive

$$
\begin{aligned}
\mathrm{e}^{\mathrm{i} \lambda \hat{a}^{\dagger} \hat{a}}\left(\hat{a}+\hat{a}^{\dagger}\right)^{n} \mathrm{e}^{-\mathrm{i} \lambda \hat{a}^{\dagger} \hat{a}} & =\left[\mathrm{e}^{\mathrm{i} \lambda \hat{a}^{\dagger} \hat{a}}\left(\hat{a}+\hat{a}^{\dagger}\right) \mathrm{e}^{-\mathrm{i} \lambda \hat{a}^{\dagger} \hat{a}}\right]^{n} \\
& =\left[\hat{a} \mathrm{e}^{-\mathrm{i} \lambda}+\hat{a}^{\dagger} \mathrm{e}^{\mathrm{i} \lambda}\right]^{n}
\end{aligned}
$$

by making use of the unitarity of $\mathrm{e}^{\mathrm{i} \lambda \hat{a}^{\dagger} \hat{a}}$. We obtain for the transformation

$$
\begin{aligned}
\mathrm{e}^{\mathrm{i} \lambda \hat{a}^{\dagger} \hat{a}} \mathrm{e}^{\mathrm{i} \xi\left(\hat{a}+\hat{a}^{\dagger}\right)} \mathrm{e}^{-\mathrm{i} \lambda \hat{a}^{\dagger} \hat{a}} & =\mathrm{e}^{\mathrm{i} \lambda \hat{a}^{\dagger} \hat{a}} \sum_{n} \frac{(\mathrm{i} \xi)^{n}}{n !}\left(\hat{a}+\hat{a}^{\dagger}\right)^{n} \mathrm{e}^{-\mathrm{i} \lambda \hat{a}^{\dagger} \hat{a}} \\
& =\sum_{n} \frac{(\mathrm{i} \xi)^{n}}{n !}\left[\hat{a} \mathrm{e}^{-\mathrm{i} \lambda}+\hat{a}^{\dagger} \mathrm{e}^{\mathrm{i} \lambda}\right]^{n} \\
& =\exp \left(\mathrm{i} \xi\left[\hat{a} \mathrm{e}^{-\mathrm{i} \lambda}+\hat{a}^{\dagger} \mathrm{e}^{\mathrm{i} \lambda}\right]\right) .
\end{aligned}
$$

\section{Appendix D. Matrix elements of displacement operator}

The following derivation is based on [95]. A difference is that we do not restrict the displacement to purely imaginary $\lambda$ in the following. A similar derivation can be found in appendix B of [111].

The simple Baker-Campbell-Hausdorff formula

$$
\mathrm{e}^{A+B}=\mathrm{e}^{A} \mathrm{e}^{B} \mathrm{e}^{-[A, B] / 2}
$$

holds for $[A,[A, B]]=[B,[B, A]]=0$.

We obtain for the annihilation operator

$$
\hat{a}^{m}|n\rangle= \begin{cases}\sqrt{\frac{n !}{(n-m) !}}|n-m\rangle & \text { for } m \leqslant n, \\ 0 & \text { else. }\end{cases}
$$

Using the above form of the Baker-Campbell-Hausdorff formula, we can rewrite the displacement operator as

$$
\hat{D}(\lambda)=\mathrm{e}^{\lambda \hat{a}^{\dagger}-\lambda^{*} \hat{a}}=\mathrm{e}^{-|\lambda|^{2} / 2} \mathrm{e}^{\lambda \hat{a}^{\dagger}} \mathrm{e}^{-\lambda^{*} \hat{a}} .
$$

With

$$
\begin{aligned}
\mathrm{e}^{-\lambda^{*} \hat{a}}|n\rangle & =\sum_{m} \frac{\left(-\lambda^{*}\right)^{m}}{m !} \hat{a}^{m}|n\rangle \\
& =\sum_{m} \frac{\left(-\lambda^{*}\right)^{m}}{m !} \sqrt{\frac{n !}{(n-m) !}}|n-m\rangle,
\end{aligned}
$$

this yields for $n^{\prime} \geqslant n$

$$
\begin{aligned}
\left\langle n^{\prime}|\hat{D}(\lambda)| n\right\rangle= & \mathrm{e}^{-|\lambda|^{2} / 2}\left\langle n^{\prime}\left|\mathrm{e}^{\lambda \hat{a}^{\dagger}} \mathrm{e}^{-\lambda^{*} \hat{a}}\right| n\right\rangle \\
= & \mathrm{e}^{-|\lambda|^{2} / 2} \sum_{m^{\prime}} \sum_{m}\left\langle n^{\prime}-m^{\prime} \mid n-m\right\rangle \frac{\lambda^{m^{\prime}}}{m^{\prime} !} \\
& \times \frac{\left(-\lambda^{*}\right)^{m}}{m !} \sqrt{\frac{n^{\prime} !}{\left(n^{\prime}-m^{\prime}\right) !} \sqrt{\frac{n !}{(n-m) !}}} \\
= & \mathrm{e}^{-|\lambda|^{2} / 2} \lambda^{n^{\prime}-n} \sum_{m=0}^{n} \frac{(-1)^{m}|\lambda|^{2 m}}{m !\left(n^{\prime}-n+m\right) !} \\
& \times \frac{\sqrt{n^{\prime} ! n !}}{(n-m) !} \\
= & \mathrm{e}^{-|\lambda|^{2} / 2} \lambda^{n^{\prime}-n} \sqrt{\frac{n !}{n^{\prime} !}} L_{n}^{\left(n^{\prime}-n\right)}\left(|\lambda|^{2}\right),
\end{aligned}
$$

where $L_{n}^{(\alpha)}(x)$ denotes the generalized Laguerre polynomials [196]. Analogously, we obtain for $n^{\prime} \leqslant n$

$$
\left\langle n^{\prime}|\hat{D}(\lambda)| n\right\rangle=\mathrm{e}^{-|\lambda|^{2} / 2}\left(-\lambda^{*}\right)^{n-n^{\prime}} \sqrt{\frac{n^{\prime} !}{n !}} L_{n^{\prime}}^{\left(n-n^{\prime}\right)}\left(|\lambda|^{2}\right) .
$$

For values $\lambda=\mathrm{i} \eta \mathrm{e}^{\mathrm{i} \omega t}$ with $\eta \in \mathbb{R}$, we can write equations (D.5) and (D.6) as

$$
\begin{aligned}
\left\langle n^{\prime}\left|\hat{D}\left(\mathrm{i} \eta \mathrm{e}^{\mathrm{i} \omega t}\right)\right| n\right\rangle= & \mathrm{e}^{-\eta^{2} / 2}(\mathrm{i} \eta)^{\left|n^{\prime}-n\right|} \mathrm{e}^{\mathrm{i} \omega\left(n^{\prime}-n\right) t} \\
& \times \sqrt{\frac{n_{<} !}{n_{>} !}} L_{n_{<}}^{\left|n^{\prime}-n\right|}\left(\eta^{2}\right),
\end{aligned}
$$

where $n_{<}:=\min \left(n^{\prime}, n\right)$ and $n_{>}:=\max \left(n^{\prime}, n\right)$.

\section{Appendix E. System of differential equations of the Rabi problem}

The Rabi problem consists of the following system of differential equations:

$$
\left|\begin{array}{c}
\dot{c}_{2}=\lambda \mathrm{e}^{-\mathrm{i} \omega t} c_{1} \\
\dot{c}_{1}=-\lambda^{*} \mathrm{e}^{\mathrm{i} \omega t} c_{2}
\end{array}\right|
$$

It can be solved by differentiating with respect to $t$

$$
\left|\begin{array}{l}
\ddot{c}_{2}=\lambda \mathrm{e}^{-\mathrm{i} \omega t} \dot{c}_{1}-\mathrm{i} \omega \lambda \mathrm{e}^{-\mathrm{i} \omega t} c_{1} \\
\ddot{c}_{1}=-\lambda^{*} \mathrm{e}^{\mathrm{i} \omega t} \dot{c}_{2}-\mathrm{i} \omega \lambda^{*} \mathrm{e}^{\mathrm{i} \omega t} c_{2}
\end{array}\right|
$$

and inserting equation (E.1):

$$
\begin{gathered}
\ddot{c}_{2}=-\mathrm{i} \omega \dot{c}_{2}-|\lambda|^{2} c_{2}, \\
\ddot{c}_{1}=\mathrm{i} \omega \dot{c}_{1}-|\lambda|^{2} c_{1} .
\end{gathered}
$$

Using the ansatz $c_{i}=a_{i} \mathrm{e}^{\mathrm{i} \kappa_{i} t}$ we obtain the characteristic equations

$$
\begin{gathered}
-\kappa_{2}^{2}=\omega \kappa_{2}-|\lambda|^{2}, \\
-\kappa_{1}^{2}=-\omega \kappa_{1}-|\lambda|^{2},
\end{gathered}
$$

which have the solutions

$$
\begin{aligned}
& \kappa_{2, \pm}=-\frac{\omega}{2} \pm \sqrt{\frac{\omega^{2}}{4}+|\lambda|^{2}}:=-\frac{\omega}{2} \pm \kappa^{\prime}, \\
& \kappa_{1, \pm}=\frac{\omega}{2} \pm \sqrt{\frac{\omega^{2}}{4}+|\lambda|^{2}}:=\frac{\omega}{2} \pm \kappa^{\prime}=-\kappa_{2, \mp} .
\end{aligned}
$$

Here, we introduced the abbreviation $\kappa^{\prime}:=\sqrt{\left(\omega^{2} / 4\right)+|\lambda|^{2}}$. The solutions of equations (E.3) and (E.4) read

$$
\begin{aligned}
c_{2} & =a_{2,+} \mathrm{e}^{\mathrm{i} \kappa_{2,+} t}+a_{2,-} \mathrm{e}^{\mathrm{i} \kappa_{2,-} t} \\
& =\left(a_{2,+} \mathrm{e}^{\mathrm{i} \kappa^{\prime} t}+a_{2,-} \mathrm{e}^{-\mathrm{i} \kappa^{\prime} t}\right) \mathrm{e}^{-\mathrm{i} \omega t / 2}, \\
c_{1} & =a_{1,+} \mathrm{e}^{\mathrm{i} \kappa_{1,+} t}+a_{1,-} \mathrm{e}^{\mathrm{i} \kappa_{1,-} t} \\
& =\left(a_{1,+} \mathrm{e}^{\mathrm{i} \kappa^{\prime} t}+a_{1,-} \mathrm{e}^{-\mathrm{i} \kappa^{\prime} t}\right) \mathrm{e}^{\mathrm{i} \omega t / 2} .
\end{aligned}
$$

Inserting them into the original system of differential equations equation (E.1), we obtain the following relations for the constants $a_{i, \pm}$ :

$$
\begin{aligned}
& \mathrm{i} \kappa_{2, \pm} a_{2, \pm}=\lambda a_{1, \pm}, \\
& \mathrm{i} \kappa_{1, \pm} a_{1, \pm}=-\lambda^{*} a_{2, \pm} .
\end{aligned}
$$


We replace $a_{1, \pm}$ by $a_{2, \pm}$ using equation (E.11) and obtain

$$
\begin{aligned}
& c_{2}=\left(a_{2,+} \mathrm{e}^{\mathrm{i} \kappa^{\prime} t}+a_{2,-} \mathrm{e}^{-\mathrm{i} \kappa^{\prime} t}\right) \mathrm{e}^{-\mathrm{i} \omega t / 2}, \\
& c_{1}=\left(\mu_{+} a_{2,+} \mathrm{e}^{\mathrm{i} \kappa^{\prime} t}+\mu_{-} a_{2,-} \mathrm{e}^{-\mathrm{i} \kappa^{\prime} t}\right) \mathrm{e}^{\mathrm{i} \omega t / 2},
\end{aligned}
$$

where we introduced the (temporary) abbreviation $\mu_{ \pm}:=$ $\mathrm{i} \kappa_{2, \pm} / \lambda$.

The constants $a_{2, \pm}$ can now be expressed in terms of the initial values $c_{20}:=c_{2}(t=0)$ and $c_{10}:=c_{1}(t=0)$. Setting $t=0$ in equations (E.13) and (E.14), we obtain a system of linear equations with the solutions

$$
\begin{aligned}
& a_{2,+}=\frac{\mu_{-} c_{20}-c_{10}}{\mu_{-}-\mu_{+}}, \\
& a_{2,-}=-\frac{\mu_{+} c_{20}-c_{10}}{\mu_{-}-\mu_{+}} .
\end{aligned}
$$

Hence,

$$
\begin{aligned}
& c_{2}=\frac{\left(\mu_{-} c_{20}-c_{10}\right) \mathrm{e}^{\mathrm{i} \kappa^{\prime} t}-\left(\mu_{+} c_{20}-c_{10}\right) \mathrm{e}^{-\mathrm{i} \kappa^{\prime} t}}{\mu_{-}-\mu_{+}} \mathrm{e}^{-\mathrm{i} \omega t / 2}, \\
& c_{1}=\frac{\mu_{+}\left(\mu_{-} c_{20}-c_{10}\right) \mathrm{e}^{\mathrm{i} \kappa^{\prime} t}-\mu_{-}\left(\mu_{+} c_{20}-c_{10}\right) \mathrm{e}^{-\mathrm{i} \kappa^{\prime} t}}{\mu_{-}-\mu_{+}} \mathrm{e}^{\mathrm{i} \omega t / 2},
\end{aligned}
$$

and by expressing $\mu_{ \pm}$in terms of $\omega, \kappa^{\prime}$, and $\lambda$ we obtain

$$
\begin{aligned}
c_{2}(t)= & \left(\cos \left(\kappa^{\prime} t\right)+\frac{\omega}{2} \frac{\mathrm{i}}{\kappa^{\prime}} \sin \left(\kappa^{\prime} t\right)\right) \mathrm{e}^{-\mathrm{i} \omega t / 2} c_{2}(0) \\
& +\frac{\lambda}{\kappa^{\prime}} \sin \left(\kappa^{\prime} t\right) \mathrm{e}^{-\mathrm{i} \omega t / 2} c_{1}(0), \\
c_{1}(t)= & -\frac{\lambda^{*}}{\kappa^{\prime}} \sin \left(\kappa^{\prime} t\right) \mathrm{e}^{\mathrm{i} \omega t / 2} c_{2}(0) \\
& +\left(\cos \left(\kappa^{\prime} t\right)-\frac{\omega}{2} \frac{\mathrm{i}}{\kappa^{\prime}} \sin \left(\kappa^{\prime} t\right)\right) \mathrm{e}^{\mathrm{i} \omega t / 2} c_{1}(0) .
\end{aligned}
$$

\section{Appendix F. Time evolution operator}

The calculation of the time evolution operator involves terms of the form

$$
\hat{\mathcal{H}}_{m}^{(i)}(t)=\mathrm{i} \xi_{m}^{(i)} \mathrm{e}^{\mathrm{i}\left(-\delta_{m} t+\varphi^{(i)}\right)} \hat{a}_{m}^{\dagger}+\text { h.c. }
$$

where $\xi_{m}^{(i)} \in \mathbb{R}$ and the total Hamiltonian reads $\hat{\mathcal{H}}(t)=$ : $\sum_{i=1}^{N} \sum_{m=1}^{3 N} \hat{\mathcal{H}}_{m}^{(i)}(t)$. (More generally, the constants $\xi_{m}^{(i)}$ represent Hermitian operators $\hat{\xi}_{m}^{(i)}$ with $\left[\hat{\xi}_{m}^{(i)}, \hat{\xi}_{n}^{(j)}\right]=$ $0 \forall i, j, m, n$.)

The commutator of two of these terms will trivially vanish for all $i, j$ and all times $t^{\prime}, t^{\prime \prime}$, if both terms belong to different modes $m \neq n$ :

$$
\left[\hat{\mathcal{H}}_{m}^{(i)}\left(t^{\prime}\right), \hat{\mathcal{H}}_{n}^{(j)}\left(t^{\prime \prime}\right)\right]=0 \quad \text { for } \quad m \neq n .
$$

However, for $m=n$, the commutators do not vanish. Using the relation

$$
\begin{aligned}
& {\left[\mathrm{e}^{\mathrm{i} \lambda} \hat{a}^{\dagger}-\mathrm{e}^{-\mathrm{i} \lambda} \hat{a}, \mathrm{e}^{\mathrm{i} \lambda^{\prime}} \hat{a}^{\dagger}-\mathrm{e}^{-\mathrm{i} \lambda^{\prime}} \hat{a}\right]} \\
& \quad=\mathrm{e}^{\mathrm{i}\left(\lambda-\lambda^{\prime}\right)}\left(\hat{a} \hat{a}^{\dagger}-\hat{a}^{\dagger} \hat{a}\right)-\mathrm{e}^{-\mathrm{i}\left(\lambda-\lambda^{\prime}\right)}\left(\hat{a} \hat{a}^{\dagger}-\hat{a}^{\dagger} \hat{a}\right) \\
& \quad=2 \mathrm{i} \sin \left(\lambda-\lambda^{\prime}\right) \hat{\mathbb{1}}
\end{aligned}
$$

yields

$$
\begin{aligned}
& {\left[\hat{\mathcal{H}}_{m}^{(i)}\left(t^{\prime}\right), \hat{\mathcal{H}}_{m}^{(j)}\left(t^{\prime \prime}\right)\right]} \\
& \quad=2 \mathrm{i} \xi_{m}^{(i)} \xi_{m}^{(j)} \sin \left(\delta_{m}\left(t^{\prime}-t^{\prime \prime}\right)-\left(\varphi^{(i)}-\varphi^{(j)}\right)\right) .
\end{aligned}
$$

The time evolution operator can be calculated using a Magnus expansion [197, 198]. As commutators with higher 'nesting level' trivially vanish, the expansion simplifies to

$$
\begin{aligned}
\hat{U}\left(t, t_{0}\right)= & \exp \left(-\frac{\mathrm{i}}{\hbar} \int_{t_{0}}^{t} \mathrm{~d} t^{\prime} \hat{\mathcal{H}}\left(t^{\prime}\right)\right. \\
& \left.-\frac{1}{2 \hbar^{2}} \int_{t_{0}}^{t} \mathrm{~d} t^{\prime} \int_{t_{0}}^{t^{\prime}} \mathrm{d} t^{\prime \prime}\left[\hat{\mathcal{H}}\left(t^{\prime}\right), \hat{\mathcal{H}}\left(t^{\prime \prime}\right)\right]\right) .
\end{aligned}
$$

The single integrals yield

$\int_{t_{0}}^{t} \mathrm{~d} t^{\prime} \hat{\mathcal{H}}_{m}^{(i)}\left(t^{\prime}\right)=-\frac{\xi_{m}^{(i)}}{\delta_{m}}\left(\mathrm{e}^{-\mathrm{i} \delta_{m}\left(t-t_{0}\right)}-1\right) \mathrm{e}^{-\mathrm{i} \delta_{m} t_{0}} \mathrm{e}^{\mathrm{i} \varphi^{(i)}} \hat{a}_{m}^{\dagger}+$ h.c.

and the double integrals of the commutators yield

$$
\begin{aligned}
\int_{t_{0}}^{t} \mathrm{~d} t^{\prime} & \int_{t_{0}}^{t^{\prime}} \mathrm{d} t^{\prime \prime}\left[\hat{\mathcal{H}}_{m}^{(i)}\left(t^{\prime}\right), \hat{\mathcal{H}}_{m}^{(j)}\left(t^{\prime \prime}\right)\right]=2 \mathrm{i} \xi_{m}^{(i)} \xi_{m}^{(j)} \\
& \times \int_{t_{0}}^{t} \mathrm{~d} t^{\prime} \int_{t_{0}}^{t^{\prime}} \mathrm{d} t^{\prime \prime} \sin \left(\delta_{m}\left(t^{\prime}-t^{\prime \prime}\right)-\left(\varphi^{(i)}-\varphi^{(j)}\right)\right) \\
= & \frac{2 \mathrm{i} \xi_{m}^{(i)} \xi_{m}^{(j)}}{\delta_{m}} \int_{t_{0}}^{t} \mathrm{~d} t^{\prime}\left[\cos \left(\varphi^{(i)}-\varphi^{(j)}\right)\right. \\
& \left.-\cos \left(\delta_{m}\left(t^{\prime}-t_{0}\right)-\left(\varphi^{(i)}-\varphi^{(j)}\right)\right)\right] \\
= & \frac{2 \mathrm{i} \xi_{m}^{(i)} \xi_{m}^{(j)}}{\delta_{m}^{2}}\left[\delta_{m}\left(t-t_{0}\right) \cos \left(\varphi^{(i)}-\varphi^{(j)}\right)\right. \\
& \left.-\sin \left(\delta_{m}\left(t-t_{0}\right)-\left(\varphi^{(i)}-\varphi^{(j)}\right)\right)-\sin \left(\varphi^{(i)}-\varphi^{(j)}\right)\right] .
\end{aligned}
$$

Note that in the time evolution operator corresponding to $\hat{\mathcal{H}}(t)$ the terms $\sin \left(\varphi^{(i)}-\varphi^{(j)}\right)=-\sin \left(\varphi^{(j)}-\varphi^{(i)}\right)$ cancel each other.

\section{Appendix G. Canonical transformation}

The unitary operator of the canonical transformation has the form

$$
\hat{U}_{\mathrm{c}}:=\mathrm{e}^{-\left(\lambda \hat{a}^{\dagger}-\lambda^{*} \hat{a}\right)} \quad \text { with } \quad \lambda:=\frac{\xi}{\kappa}
$$

and is applied to

$$
\hat{\mathcal{H}}:=\underbrace{\left(\xi \hat{a}^{\dagger}+\xi^{*} \hat{a}\right)}_{:=\hat{\mathcal{H}}_{1}}+\underbrace{\left(-\kappa \hat{a}^{\dagger} \hat{a}\right)}_{:=\hat{\mathcal{H}}_{2}} \rightarrow \quad \hat{\mathcal{H}}^{\prime}:=\hat{U}_{\mathrm{c}} \hat{\mathcal{H}} \hat{U}_{\mathrm{c}}^{\dagger} .
$$

Here, the constants $\xi \in \mathbb{C}$ and $\kappa \in \mathbb{R}$.

We use the Baker-Campbell-Hausdorff formula from appendix $\mathrm{B}$ to do the transformation. The commutators for $\hat{\mathcal{H}}_{1}$ yield

$$
\begin{aligned}
{\left[\xi \hat{a}^{\dagger}+\xi^{*} \hat{a}, \lambda \hat{a}^{\dagger}-\lambda^{*} \hat{a}\right]=} & \xi \lambda^{*}\left(-\hat{a}^{\dagger} \hat{a}+\hat{a} \hat{a}^{\dagger}\right) \\
& -\xi^{*} \lambda\left(\hat{a}^{\dagger} \hat{a}-\hat{a} \hat{a}^{\dagger}\right) \\
= & \left(\xi \lambda^{*}+\xi^{*} \lambda\right) \hat{\mathbb{1}} \\
= & \frac{2 \xi \xi^{*}}{\kappa} \hat{\mathbb{1}} .
\end{aligned}
$$


Commutators with higher 'nesting levels' trivially vanish. Hence, the complete transformation of $\hat{\mathcal{H}}_{1}$ reads

$$
\hat{\mathcal{H}}_{1}^{\prime}:=\hat{U}_{\mathrm{c}} \hat{\mathcal{H}}_{1} \hat{U}_{\mathrm{c}}^{\dagger}=\left(\xi \hat{a}^{\dagger}+\xi^{*} \hat{a}\right)+\frac{2 \xi \xi^{*}}{\kappa} \hat{\mathbb{1}}
$$

The commutators for $\hat{\mathcal{H}}_{2}$ read

$$
\begin{aligned}
{\left[-\kappa \hat{a}^{\dagger} \hat{a}, \lambda \hat{a}^{\dagger}-\lambda^{*} \hat{a}\right]=} & -\kappa\left(\hat{a}^{\dagger}\left[\hat{a}, \lambda \hat{a}^{\dagger}-\lambda^{*} \hat{a}\right]\right. \\
& \left.+\left[\hat{a}^{\dagger}, \lambda \hat{a}^{\dagger}-\lambda^{*} \hat{a}\right] \hat{a}\right) \\
= & -\kappa\left(\lambda \hat{a}^{\dagger}+\lambda^{*} \hat{a}\right) \\
= & -\left(\xi \hat{a}^{\dagger}+\xi^{*} \hat{a}\right)
\end{aligned}
$$

and

$$
\begin{aligned}
{\left[-\kappa \hat{a}^{\dagger} \hat{a}, \lambda \hat{a}^{\dagger}-\lambda^{*} \hat{a}\right]^{\{2\}} } & =\left[-\left(\xi \hat{a}^{\dagger}+\xi^{*} \hat{a}\right), \lambda \hat{a}^{\dagger}-\lambda^{*} \hat{a}\right] \\
& =-\frac{2 \xi \xi^{*}}{\kappa} \hat{\mathbb{1}},
\end{aligned}
$$

where we used equation (G.3). Higher order terms in the expansion trivially vanish again. The complete transformation of $\hat{\mathcal{H}}_{2}$ reads

$$
\hat{\mathcal{H}}_{2}^{\prime}:=\hat{U}_{\mathrm{c}} \hat{\mathcal{H}}_{2} \hat{U}_{\mathrm{c}}^{\dagger}=-\kappa \hat{a}^{\dagger} \hat{a}-\left(\xi \hat{a}^{\dagger}+\xi^{*} \hat{a}\right)-\frac{\xi \xi^{*}}{\kappa} \hat{\mathbb{1}} .
$$

Hence, the transformation of the full Hamiltonian reads

$$
\hat{\mathcal{H}}^{\prime}=\hat{\mathcal{H}}_{1}^{\prime}+\hat{\mathcal{H}}_{2}^{\prime}=\frac{\xi \xi^{*}}{\kappa} \hat{\mathbb{1}}-\kappa \hat{a}^{\dagger} \hat{a} .
$$

We want to stress that the canonical transformation is exact in this case.

\section{References}

[1] Lloyd S 1996 Universal quantum simulators Science 273 1073-8

[2] Schinarakis K 2011 Weltrekord: Jülicher Supercomputer simuliert Quantencomputer Press release http://www2.fz-juelich.de/portal/index.php?cmd= show\&mid $=760 \&$ index $=163$

[3] De Raedt K, Michielsen K, De Raedt H, Trieu B, Arnold G, Richter M, Lippert Th, Watanabe H and Ito N 2007 Massively parallel quantum computer simulator Comput. Phys. Commun. 176 121-36

[4] Moore G E 1965 Cramming more components onto integrated circuits Electronics 38 114-7

[5] Feynman R 1982 Simulating physics with computers Int. J. Theor. Phys. 21 467-88

[6] diVincenzo D P 1995 Two-bit gates are universal for quantum computation Phys. Rev. A 51 1015-22

[7] diVincenzo D P 2000 The physical implementation of quantum computation Fortschr. Phys. 48 771-83

[8] Ladd T D, Jelezko F, Laflamme R, Nakamura Y, Monroe C and O'Brien J L 2010 Quantum computers Nature 464 45-53

[9] Nielsen M A and Chuang I L 2000 Quantum Computation and Quantum Information (Cambridge Series on Information and the Natural Sciences) (Cambridge: Cambridge University Press)

[10] Knill E 2005 Quantum computing with realistically noisy devices Nature 434 39-44

[11] Home J P, Hanneke D, Jost J D, Amini J M, Leibfried D and Wineland D J 2009 Complete methods set for scalable ion trap quantum information processing Science 325 1227-30
[12] Buluta I and Nori F 2009 Quantum simulators Science 326 108-111

[13] Hauke P, Cucchietti F M, Tagliacozzo L, Lewenstein M and Deutsch I 2011 On the robustness of quantum simulations arXiv: $1109.6457 \mathrm{v} 1$

[14] Engel G S, Calhoun T R, Read E L, Ahn T-K, Mančal T, Cheng Y-C, Blankenship R E and Fleming G R 2007 Evidence for wavelike energy transfer through quantum coherence in photosynthetic systems Nature 446 782-6

[15] Mohseni M, Rebentrost P, Lloyd S and Aspuru-Guzik A 2008 Environment-assisted quantum walks in photosynthetic energy transfer J. Chem. Phys. 129174106

[16] Leibfried D 2010 Could a boom in technologies trap Feynman's simulator? Nature $\mathbf{4 6 3} 608$

[17] Jaksch D and Zoller P 2005 The cold atom Hubbard toolbox Ann. Phys. 315 52-79

[18] Lewenstein M, Sanpera A, Ahufinger V, Damski B, Sen(De) A and Sen U 2007 Ultracold atomic gases in optical lattices: mimicking condensed matter physics and beyond Adv. Phys. $56243-379$

[19] Bloch I, Dalibard J and Zwerger W 2008 Many-body physics with ultracold gases Rev. Mod. Phys. 80 885-964

[20] Simon J, Bakr W S, Ma R, Tai M E, Preiss P M and Greiner M 2011 Quantum simulation of antiferromagnetic spin chains in an optical lattice Nature 472 307-12

[21] Porras D and Cirac J I 2004 Effective quantum spin systems with trapped ions Phys. Rev. Lett. 92207901

[22] Porras D and Cirac J I 2004 Bose-Einstein condensation and strong-correlation behavior of phonons in ion traps Phys. Rev. Lett. 93263602

[23] Wunderlich C 2002 Conditional spin resonance with trapped ions Laser Physics at the Limits ed Figger $\mathrm{H}$ et al (Berlin: Springer) pp 261-73

[24] Cirac J I and Zoller P 1995 Quantum computations with cold trapped ions Phys. Rev. Lett. 74 4091-4

[25] Chou C W, Hume D B, Koelemeij J C J, Wineland D J and Rosenband T 2010 Frequency comparison of two high-accuracy $\mathrm{Al}^{+}$optical clocks Phys. Rev. Lett. 104070802

[26] Schützhold R, Uhlmann M, Petersen L, Schmitz H, Friedenauer A and Schätz T 2007 Analogue of cosmological particle creation in an ion trap Phys. Rev. Lett. 99201301

[27] Horstmann B, Reznik B, Fagnocchi S and Cirac J I 2010 Hawking radiation from an acoustic black hole on an ion ring Phys. Rev. Lett. 104250403

[28] Horstmann B, Schützhold R, Reznik B, Fagnocchi S and Cirac J I 2011 Hawking radiation on an ion ring in the quantum regime New J. Phys. 13045008

[29] Lamata L, León J, Schätz T and Solano E 2007 Dirac equation and quantum relativistic effects in a single trapped ion Phys. Rev. Lett. 98253005

[30] Gerritsma R, Kirchmair G, Zahringer F, Solano E, Blatt R and Roos C F 2010 Quantum simulation of the Dirac equation Nature 463 68-71

[31] Casanova J, García-Ripoll J J, Gerritsma R, Roos C F and Solano E 2010 Klein tunneling and Dirac potentials in trapped ions Phys. Rev. A 82020101

[32] Gerritsma R, Lanyon B P, Kirchmair G, Zähringer F, Hempel C, Casanova J, García-Ripoll J J, Solano E, Blatt R and Roos C F 2011 Quantum simulation of the Klein paradox with trapped ions Phys. Rev. Lett. 106060503

[33] Casanova J, Sabín C, León J, Egusquiza I L, Gerritsma R, Roos C F, García-Ripoll J J and Solano E 2011 Quantum simulation of the Majorana equation and unphysical operations arXiv: $1102.1651 \mathrm{v} 2$

[34] Lamata L, Casanova J, Gerritsma R, Roos C F, García-Ripoll J-J and Solano E 2011 Relativistic quantum mechanics with trapped ions New J. Phys. 13095003 
[35] Casanova J, Lamata L, Egusquiza I L, Gerritsma R, Roos C F, García-Ripoll J J and Solano E 2011 Quantum simulation of quantum field theories in trapped ions arXiv: $1107.5233 \mathrm{v} 2$

[36] Leibfried D et al 2002 Trapped-ion quantum simulator: experimental application to nonlinear interferometers Phys. Rev. Lett. 89247901

[37] Travaglione B C and Milburn G J 2002 Implementing the quantum random walk Phys. Rev. A 65032310

[38] Schmitz H, Matjeschk R, Schneider Ch, Glueckert J, Enderlein M, Huber T and Schaetz T 2009 Quantum walk of a trapped ion in phase space Phys. Rev. Lett. 103090504

[39] Zähringer F, Kirchmair G, Gerritsma R, Solano E, Blatt R and Roos C F 2010 Realization of a quantum walk with one and two trapped ions Phys. Rev. Lett. 104100503

[40] Matjeschk R, Schneider C, Enderlein M, Huber T, Schmitz H, Glueckert J and Schaetz T 2011 Experimental simulation and limitations of quantum walks with trapped ions arXiv: $1108.0913 \mathrm{v} 1$

[41] Cai J, Guerreschi G G and Briegel H J 2010 Quantum control and entanglement in a chemical compass Phys. Rev. Lett. 104220502

[42] Cai J, Popescu S and Briegel H J 2010 Dynamic entanglement in oscillating molecules and potential biological implications Phys. Rev. E 82021921

[43] Plenio M B and Huelga S F 2008 Dephasing-assisted transport: quantum networks and biomolecules New J. Phys. 10113019

[44] Porras D, Marquardt F, von Delft J and Cirac J I 2008 Mesoscopic spin-boson models of trapped ions Phys. Rev. A 78010101

[45] Sachdev S 1999 Quantum Phase Transitions (Cambridge: Cambridge University Press)

[46] Johanning M, Varón A and Wunderlich C 2009 Quantum simulations with cold trapped ions J. Phys. B: At. Mol. Opt. Phys. 42154009

[47] Friedenauer A, Schmitz H, Glueckert J T, Porras D and Schaetz T 2008 Simulating a quantum magnet with trapped ions Nature Phys. 4 757-61

[48] Kim K, Chang M-S, Korenblit S, Islam R, Edwards E E Freericks J K, Lin G-D, Duan L-M and Monroe C 2010 Quantum simulation of frustrated Ising spins with trapped ions Nature 465 590-3

[49] Islam R, Edwards E E, Kim K, Korenblit S, Noh C, Carmichael H, Lin G-D, Duan L-M, Wang C-C J, Freericks J K and Monroe C 2011 Onset of a quantum phase transition with a trapped ion quantum simulator Nature Commun. 2377

[50] Wineland D J, Monroe C, Itano W M, Leibfried D, King B E and Meekhof D M 1998 Experimental issues in coherent quantum-state manipulation of trapped atomic ions J. Res. Natl Inst. Stand. Technol. 103 259-328

[51] Leibfried D, Blatt R, Monroe C and Wineland D 2003 Quantum dynamics of single trapped ions Rev. Mod. Phys. $75281-324$

[52] Biercuk M J, Uys H, VanDevender A P, Shiga N, Itano W M and Bollinger J J 2009 High-fidelity quantum control using ion crystals in a Penning trap Quantum Inform. Comput. 9 0920-0949

[53] Bollinger $\mathrm{J}$ and Britton $\mathrm{J} 2011$ private communication

[54] Paul W 1990 Electromagnetic traps for charged and neutral particles Rev. Mod. Phys. 62 531-40

[55] Dehmelt H 1990 Experiments with an isolated subatomic particle at rest Rev. Mod. Phys. 62 525-30

[56] Phillips W D 1998 Nobel lecture: laser cooling and trapping of neutral atoms Rev. Mod. Phys. 70 721-41
[57] Paul W and Steinwedel H 1953 Ein neues massenspektrometer ohne magnetfeld $Z$. Naturf. a $8448-50$

[58] Paul W, Osberghaus O and Fischer E 1958 Ein Ionenkäfig Forschungsber. Wirtsch. Verkehrsministeriums Nordrhein-Westfalen 415 1-42

[59] Ashkin A 1970 Acceleration and trapping of particles by radiation pressure Phys. Rev. Lett. 24 156-9

[60] Cormick C, Schaetz T and Morigi G 2011 Trapping ions with lasers New J. Phys. 13043019

[61] Wineland D J and Dehmelt H 1975 Proposed $10^{14} \delta v<v$ laser fluorescence spectroscopy on $\mathrm{T}^{+}$mono-ion oscillator III Bull. Am. Phys. Soc. 20637

[62] Hänsch T W and Schawlow A L 1975 Cooling of gases by laser radiation Opt. Commun. 13 68-9

[63] Wineland D J, Drullinger R E and Walls F L 1978 Radiation-pressure cooling of bound resonant absorbers Phys. Rev. Lett. 40 1639-42

[64] Neuhauser W, Hohenstatt M, Toschek P and Dehmelt H 1978 Optical-sideband cooling of visible atom cloud confined in parabolic well Phys. Rev. Lett. 41 233-6

[65] Waki I, Kassner S, Birkl G and Walther H 1992 Observation of ordered structures of laser-cooled ions in a quadrupole storage ring Phys. Rev. Lett. 68 2007-10

[66] Birkl G, Kassner S and Walther H 1992 Multiple-shell structures of laser-cooled ${ }^{24} \mathrm{Mg}^{+}$ions in a quadrupole storage ring Nature 357 310-13

[67] Monz T, Schindler P, Barreiro J T, Chwalla M, Nigg D, Coish W A, Harlander M, Hänsel W, Hennrich M and Blatt R 2011 14-qubit entanglement: creation and coherence Phys. Rev. Lett. 106130506

[68] Lin G-D, Zhu S-L, Islam R, Kim K, Chang M-S, Korenblit S, Monroe C and Duan L-M 2009 Large-scale quantum computation in an anharmonic linear ion trap Europhys. Lett. 8660004

[69] Schätz T, Schramm U and Habs D 2001 Crystalline ion beams Nature 412 717-20

[70] Schramm U, Schätz T and Habs D 2001 Bunched crystalline ion beams Phys. Rev. Lett. 87184801

[71] Schramm U, Schätz T and Habs D 2002 Three-dimensional crystalline ion beams Phys. Rev. E 66036501

[72] Moehring D L 2011 private communication

[73] Chiaverini J, Blakestad R B, Britton J, Jost J D, Langer C, Leibfried D, Ozeri R and Wineland D J 2005 Surface-electrode architecture for ion-trap quantum information processing Quantum Inform. Comput. 5 419-39

[74] Seidelin S et al 2006 Microfabricated surface-electrode ion trap for scalable quantum information processing Phys. Rev. Lett. 96253003

[75] Kielpinski D, Monroe C and Wineland D J 2002 Architecture for a large-scale ion-trap quantum computer Nature 417 709-11

[76] Tan J N, Bollinger J J, Jelenkovic B and Wineland D J 1995 Long-range order in laser-cooled, atomic-ion Wigner crystals observed by Bragg scattering Phys. Rev. Lett. 75 4198-201

[77] Itano W M, Bollinger J J, Tan J N, Jelenković B, Huang X-P and Wineland D J 1998 Bragg diffraction from crystallized ion plasmas Science 279 686-9

[78] Mitchell T B, Bollinger J J, Dubin D H E, Huang X-P, Itano W M and Baughman R H 1998 Direct observations of structural phase transitions in planar crystallized ion plasmas Science 282 1290-3

[79] Porras D and Cirac J I 2006 Quantum manipulation of trapped ions in two dimensional Coulomb crystals Phys. Rev. Lett. 96250501

[80] Schneider Ch, Enderlein M, Huber T and Schaetz T 2010 Optical trapping of an ion Nature Photon. 4 772-5 
[81] Trapped Ion Quantum Information Group; University of Maryland. Ion periodic table (http://www.iontrap. umd.edu/research_info/ioncatalog/index.html)

[82] Saffman M and Mølmer K 2008 Scaling the neutral-atom Rydberg gate quantum computer by collective encoding in holmium atoms Phys. Rev. A 78012336

[83] Bollinger J J, Heizen D J, Itano W M, Gilbert S L and Wineland D J 1991 A 303-Mhz frequency standard based on trapped $\mathrm{Be}^{+}$ions IEEE Trans. Instrum. Meas. 40 126-8

[84] Fisk P T H, Sellars M J, Lawn M A, Coles C, Mann A G and Blair D G 1995 Very high Q microwave spectroscopy on trapped ${ }^{171} \mathrm{Yb}^{+}$ions: application as a frequency standard IEEE Trans. Instrum. Meas. 44 113-6

[85] Sørensen A and Mølmer K 1999 Quantum computation with ions in thermal motion Phys. Rev. Lett. 82 1971-4

[86] Roos C F 2008 Ion trap quantum gates with amplitude-modulated laser beams New J. Phys. 10013002

[87] Lee P J, Brickman K-A, Deslauriers L, Haljan P C, Duan L-M and Monroe C 2005 Phase control of trapped ion quantum gates J. Opt. B 7 S371-83

[88] Mintert F and Wunderlich C 2001 Ion-trap quantum logic using long-wavelength radiation Phys. Rev. Lett. 87257904

[89] Johanning M, Braun A, Timoney N, Elman V, Neuhauser W and Wunderlich C 2009 Individual addressing of trapped ions and coupling of motional and spin states using $\mathrm{rf}$ radiation Phys. Rev. Lett. 102073004

[90] Timoney N, Elman V, Glaser S, Weiss C, Johanning M, Neuhauser W and Wunderlich C 2008 Error-resistant single-qubit gates with trapped ions Phys. Rev. A 77052334

[91] Ospelkaus C, Langer C E, Amini J M, Brown K R, Leibfried D and Wineland D J 2008 Trapped-ion quantum logic gates based on oscillating magnetic fields Phys. Rev Lett. 101090502

[92] Brown K R, Wilson A C, Colombe Y, Ospelkaus C, Meier A M, Knill E, Leibfried D and Wineland D J 2011 Single-qubit-gate error below $10^{-4}$ in a trapped ion Phys. Rev. A 84030303

[93] Ospelkaus C, Warring U, Colombe Y, Brown K R, Amini J M, Leibfried D and Wineland D J 2011 Microwave quantum logic gates for trapped ions Nature 476 181-4

[94] Happer W 1972 Optical pumping Rev. Mod. Phys. 44 169-250

[95] Wineland D J and Itano W M 1979 Laser cooling of atoms Phys. Rev. A 20 1521-40

[96] Diedrich F, Bergquist J C, Itano W M and Wineland D J 1989 Laser cooling to the zero-point energy of motion Phys. Rev. Lett. 62 403-6

[97] Monroe C, Meekhof D M, King B E, Jefferts S R, Itano W M, Wineland D J and Gould P 1995 Resolved-sideband Raman cooling of a bound atom to the 3D zero-point energy Phys. Rev. Lett. 75 4011-4

[98] Morigi G, Eschner J and Keitel C H 2000 Ground state laser cooling using electromagnetically induced transparency Phys. Rev. Lett. 85 4458-61

[99] Roos C F, Leibfried D, Mundt A, Schmidt-Kaler F, Eschner J and Blatt R 2000 Experimental demonstration of ground state laser cooling with electromagnetically induced transparency Phys. Rev. Lett. 85 5547-50

[100] Wineland D J, Bergquist J C, Itano W M and Drullinger R E 1980 Double-resonance and optical-pumping experiments on electromagnetically confined, laser-cooled ions $O p t$. Lett. $5245-7$

[101] Nagourney W, Sandberg J and Dehmelt H 1986 Shelved optical electron amplifier: observation of quantum jumps Phys. Rev. Lett. 56 2797-9
[102] Sauter Th, Neuhauser W, Blatt R and Toschek P E 1986 Observation of quantum jumps Phys. Rev. Lett. 57 1696-8

[103] Bergquist J C, Hulet R G, Itano W M and Wineland D J 1986 Observation of quantum jumps in a single atom Phys. Rev. Lett. 57 1699-702

[104] Myerson A H, Szwer D J, Webster S C, Allcock D T C, Curtis M J, Imreh G, Sherman J A, Stacey D N, Steane A M and Lucas D M 2008 High-fidelity readout of trapped-ion qubits Phys. Rev. Lett. 100200502

[105] Burrell A H, Szwer D J, Webster S C and Lucas D M 2010 Scalable simultaneous multiqubit readout with $99.99 \%$ single-shot fidelity Phys. Rev. A 81040302

[106] Schaetz T, Barrett M D, Leibfried D, Britton J, Chiaverini J, Itano W M, Jost J D, Knill E, Langer C and Wineland D J 2005 Enhanced quantum state detection efficiency through quantum information processing Phys. Rev. Lett. 94010501

[107] Hume D B, Rosenband T and Wineland D J 2007 High-fidelity adaptive qubit detection through repetitive quantum nondemolition measurements Phys. Rev. Lett. 99120502

[108] Schmidt P O, Rosenband T, Langer C, Itano W M, Bergquist J C and Wineland D J 2005 Spectroscopy using quantum logic Science 309 749-52

[109] Meekhof D M, Monroe C, King B E, Itano W M and Wineland D J 1996 Generation of nonclassical motional states of a trapped atom Phys. Rev. Lett. 76 1796-9

[110] Leibfried D et al 2003 Experimental demonstration of a robust, high-fidelity geometric two ion-qubit phase gate Nature 422 412-5

[111] Cahill K E and Glauber R J 1969 Ordered expansions in boson amplitude operators Phys. Rev. 177 1857-81

[112] Berry M V 1984 Quantal phase factors accompanying adiabatic changes Proc. R. Soc. Lond. A 392 45-57

[113] Aharonov Y and Anandan J 1987 Phase change during a cyclic quantum evolution Phys. Rev. Lett. 58 1593-6

[114] Solano E, de Matos Filho R L and Zagury N 1999 Deterministic Bell states and measurement of the motional state of two trapped ions Phys. Rev. A 59 R2539-R2543

[115] Milburn G J, Schneider S and James D F V 2000 Ion trap quantum computing with warm ions Fortschr. Phys. 48 801-10

[116] Sørensen A and Mølmer K 2000 Entanglement and quantum computation with ions in thermal motion Phys. Rev. A 62022311

[117] Wang X, Sørensen A and Mølmer K 2001 Multibit gates for quantum computing Phys. Rev. Lett. 86 3907-10

[118] Home J P, McDonnell M J, Lucas D M, Imreh G, Keitch B C, Szwer D J, Thomas N R, Webster S C, Stacey D N and Steane A M 2006 Deterministic entanglement and tomography of ion-spin qubits New J. Phys. 8188

[119] Sackett C A et al 2000 Experimental entanglement of four particles Nature 404 256-9

[120] Haljan P C, Lee P J, Brickman K-A, Acton M, Deslauriers L and Monroe C 2005 Entanglement of trapped-ion clock states Phys. Rev. A 72062316

[121] Benhelm J, Kirchmair G, Roos C F and Blatt R 2008 Towards fault-tolerant quantum computing with trapped ions Nature Phys. 4 463-6

[122] Kim K, Chang M-S, Islam R, Korenblit S, Duan L-M and Monroe C 2009 Entanglement and tunable spin-spin couplings between trapped ions using multiple transverse modes Phys. Rev. Lett. 103120502

[123] Schmitz H, Friedenauer A, Schneider C, Matjeschk R, Enderlein M, Huber T, Glueckert J, Porras D and Schaetz T 2009 The 'arch' of simulating quantum spin systems with trapped ions Appl. Phys. B 95 195-203 
[124] Porras D and Cirac J I 2008 Quantum manipulation of trapped ions in two dimensional Coulomb crystals arXiv:quant-ph/0601148v3

[125] Deng X-L, Porras D and Cirac J I 2005 Effective spin quantum phases in systems of trapped ions Phys. Rev. A 72063407

[126] Bermudez A, Schaetz T and Porras D 2011 Synthetic gauge fields for vibrational excitations of trapped ions Phys. Rev. Lett. 107150501

[127] Landa H, Marcovitch S, Retzker A, Plenio M B and Reznik B 2010 Quantum coherence of discrete kink solitons in ion traps Phys. Rev. Lett. 104043004

[128] Sachdev S 2000 Quantum criticality: competing ground states in low dimensions Science 288 475-80

[129] Blatt R 2011 private communication

[130] Hauke P, Cucchietti F M, Müller-Hermes A, Bañuls M-C, Cirac J I and Lewenstein M 2010 Complete devil's staircase and crystal-superfluid transitions in a dipolar $X X Z$ spin chain: a trapped ion quantum simulation New $J$. Phys. 12113037

[131] Schmied R, Wesenberg J H and Leibfried D 2011 Quantum simulation of the hexagonal Kitaev model with trapped ions New J. Phys. 13115011

[132] Shimshoni E, Morigi G and Fishman S 2011 Quantum zigzag transition in ion chains Phys. Rev. Lett. 106010401

[133] Shimshoni E, Morigi G and Fishman S 2011 Quantum structural phase transition in chains of interacting atoms Phys. Rev. A 83032308

[134] Milman P, Maineult W, Guibal S, Guidoni L, Douçot B, Ioffe L and Coudreau T 2007 Topologically decoherence-protected qubits with trapped ions Phys. Rev Lett. 99020503

[135] Bermudez A, Porras D and Martin-Delgado M A 2009 Competing many-body interactions in systems of trapped ions Phys. Rev. A 79060303

[136] Barreiro J T, Müller M, Schindler P, Nigg D, Monz T, Chwalla M, Hennrich M, Roos C F, Zoller P and Blatt R 2011 An open-system quantum simulator with trapped ions Nature 470 486-91

[137] Deng X-L, Porras D and Cirac J I 2008 Quantum phases of interacting phonons in ion traps Phys. Rev. A 77033403

[138] Brown K R, Ospelkaus C, Colombe Y, Wilson A C, Leibfried D and Wineland D J 2011 Coupled quantized mechanical oscillators Nature 471 196-9

[139] Harlander M, Lechner R, Brownnutt M, Blatt R and Hänsel W 2011 Trapped-ion antennae for the transmission of quantum information Nature 471 200-3

[140] Bermudez A, Martin-Delgado M A and Porras D 2010 The localization of phonons in ion traps with controlled quantum disorder New J. Phys. 12123016

[141] Ivanov P A, Ivanov S S, Vitanov N V, Mering A, Fleischhauer M and Singer K 2009 Simulation of a quantum phase transition of polaritons with trapped ions Phys. Rev. A 80060301

[142] Ivanov P A, Vitanov N V, Singer K and Schmidt-Kaler F 2010 Dynamical control and novel quantum phases in impurity doped linear ion crystals arXiv: $1002.3033 \mathrm{v} 2$

[143] Ivanov P A and Schmidt-Kaler F 2011 Simulation of quantum magnetism in mixed spin systems with impurity doped ion crystal arXiv: $1105.0598 \mathrm{v} 1$

[144] Kibble T W B 1976 Topology of cosmic domains and strings J. Phys. A: Math. Gen. $91387-98$

[145] Zurek W H 1985 Cosmological experiments in superfluid helium? Nature 317 505-8

[146] del Campo A, De Chiara G, Morigi G, Plenio M B and Retzker A 2010 Structural defects in ion chains by quenching the external potential: the inhomogeneous Kibble-Zurek mechanism Phys. Rev. Lett. 105075701
[147] Marcovitch S and Reznik B 2008 Entanglement of solitons in the Frenkel-Kontorova model Phys. Rev. A 78052303

[148] Kartashov Y V, Malomed B A and Torner L 2011 Solitons in nonlinear lattices Rev. Mod. Phys. 83 247-305

[149] Eisenberg H S, Silberberg Y, Morandotti R, Boyd A R and Aitchison J S 1998 Discrete spatial optical solitons in waveguide arrays Phys. Rev. Lett. 81 3383-6

[150] Fleischer J W, Segev M, Efremidis N K and Christodoulides D N 2003 Observation of two-dimensional discrete solitons in optically induced nonlinear photonic lattices Nature 422 147-150

[151] Trombettoni A and Smerzi A 2001 Discrete solitons and breathers with dilute Bose-Einstein condensates Phys. Rev. Lett. 86 2353-6

[152] Frenkel J and Kontorowa T 1938 On the theory of plastic deformation and twinning Phys. Z. Sowjetunion $131-10$

[153] Kivshar Y S and Braun O M 2004 The Frenkel-Kontorova Model (Berlin: Springer)

[154] García-Mata I, Zhirov O V and Shepelyansky D L 2007 Frenkel-Kontorova model with cold trapped ions Eur. Phys. J. D 41 325-30

[155] Benassi A, Vanossi A and Tosatti E 2011 Nanofriction in cold ion traps Nature Commun. 2236

[156] Pruttivarasin T, Ramm M, Talukdar I, Kreuter A and Häffner H 2011 Trapped ions in optical lattices for probing oscillator chain models New J. Phys. 13075012

[157] Schmied R, Roscilde T, Murg V, Porras D and Cirac J I 2008 Quantum phases of trapped ions in an optical lattice $\mathrm{New}$ J. Phys. 10045017

[158] Folman R, Krüger P, Cassettari D, Hessmo B, Maier T and Schmiedmayer J 2000 Controlling cold atoms using nanofabricated surfaces: atom chips Phys. Rev. Lett. 84 4749-52

[159] Stick D, Fortier K M, Haltli R, Highstrete C, Moehring D L, Tigges C and Blain M G 2010 Demonstration of a microfabricated surface electrode ion trap arXiv: 1008.0990v2

[160] Moehring D L, Highstrete C, Stick D, Fortier K M, Haltli R, Tigges C and Blain M G 2011 Design, fabrication and experimental demonstration of junction surface ion traps New J. Phys. 13075018

[161] Turchette Q A et al 2000 Heating of trapped ions from the quantum ground state Phys. Rev. A 61063418

[162] Labaziewicz J, Ge Y, Antohi P, Leibrandt D, Brown K R and Chuang I L 2008 Suppression of heating rates in cryogenic surface-electrode ion traps Phys. Rev. Lett. 100013001

[163] Wineland D J and Leibfried D 2011 Quantum information processing and metrology with trapped ions Laser Phys. Lett. 8 175-88

[164] Schaetz T, Friedenauer A, Schmitz H, Petersen L and Kahra S 2007 Towards (scalable) quantum simulations in ion traps J. Mod. Opt. 54 2317-25

[165] Schmied R, Wesenberg J H and Leibfried D 2009 Optimal surface-electrode trap lattices for quantum simulation with trapped ions Phys. Rev. Lett. 102233002

[166] Kumph M, Brownnutt M and Blatt R 2011 Two-dimensional arrays of radio-frequency ion traps with addressable interactions New J. Phys. 13073043

[167] Karin T, Le Bras I, Kehlberger A, Singer K, Daniilidis N and Häffner H 2012 Transport of charged particles by adjusting rf voltage amplitudes Appl. Phys. B at press (arXiv:1011.6116v5)

[168] Chiaverini J and Lybarger W E 2008 Laserless trapped-ion quantum simulations without spontaneous scattering using microtrap arrays Phys. Rev. A 77022324

[169] Hennsinger W 2011 private communication 
[170] Castrejón-Pita J R, Ohadi H, Crick D R, Winters D F A, Segal D M and Thompson R C 2007 Novel designs for Penning ion traps J. Mod. Opt. 54 1581-94

[171] Hellwig M, Bautista-Salvador A, Singer K, Werth G and Schmidt-Kaler F 2010 Fabrication of a planar micro Penning trap and numerical investigations of versatile ion positioning protocols New J. Phys. 12065019

[172] Schmied R 2010 Electrostatics of gapped and finite surface electrodes New J. Phys. 12023038

[173] Hurst G S, Payne M G, Kramer S D and Young J P 1979 Resonance ionization spectroscopy and one-atom detection Rev. Mod. Phys. 51 767-819

[174] Kjærgaard N, Hornekær L, Thommesen A M, Videsen Z and Drewsen M 2000 Isotope selective loading of an ion trap using resonance-enhanced two-photon ionization Appl. Phys. B 71 207-10

[175] Pearson C E, Leibrandt D R, Bakr W S, Mallard W J, Brown K R and Chuang I L 2006 Experimental investigation of planar ion traps Phys. Rev. A 73032307

[176] Allcock D T C et al 2010 Implementation of a symmetric surface-electrode ion trap with field compensation using a modulated Raman effect New J. Phys. 12053026

[177] Berkeland D J, Miller J D, Bergquist J C, Itano W M and Wineland D J 1998 Minimization of ion micromotion in a Paul trap J. Appl. Phys. 83 5025-33

[178] Friedenauer A, Markert F, Schmitz H, Petersen L, Kahra S, Herrmann M, Udem T, Hänsch T W and Schätz T 2006 High power all solid state laser system near $280 \mathrm{~nm} \mathrm{Appl}$. Phys. B 84 371-3

[179] Bertelsen A, Vogelius I S, Jørgensen S, Kosloff R and Drewsen M 2004 Photo-dissociation of cold $\mathrm{MgH}^{+}$Eur. Phys. J. D 31 403-8

[180] Cetina M, Grier A, Campbell J, Chuang I and Vuletić V 2007 Bright source of cold ions for surface-electrode traps Phys. Rev. A 76041401

[181] Feng Y, Taylor L R and Calia D B $2009150 \mathrm{~W}$ highly-efficient Raman fiber laser Opt. Express 17 23678-83

[182] Feng Y, Taylor L R and Calia D B 200925 W Raman-fiber-amplifier-based $589 \mathrm{~nm}$ laser for laser guide star Opt. Express 17 19021-6

[183] Toptica Photonics AG 2010 private communication

[184] Dumke R, Volk M, Müther T, Buchkremer F B J, Birkl G and Ertmer W 2002 Micro-optical realization of arrays of selectively addressable dipole traps: a scalable configuration for quantum computation with atomic qubits Phys. Rev. Lett. 89097903

[185] Streed E W, Norton B G, Jechow A, Weinhold T J and Kielpinski D 2011 Imaging of trapped ions with a microfabricated optic for quantum information processing Phys. Rev. Lett. 106010502

[186] VanDevender A P, Colombe Y, Amini J, Leibfried D and Wineland D J 2010 Efficient fiber optic detection of trapped ion fluorescence Phys. Rev. Lett. 10502300

[187] Shu G, Kurz N, Dietrich M R and Blinov B B 2010 Efficient fluorescence collection from trapped ions with an integrated spherical mirror Phys. Rev. A 81042321

[188] Noek R, Knoernschild C, Migacz J, Kim T, Maunz P, Merrill T, Hayden H, Pai C S and Kim J 2010 Multiscale optics for enhanced light collection from a point source Opt. Lett. 35 2460-2

[189] Herskind P F, Wang S X, Shi M, Ge Y, Cetina M and Chuang I L 2011 Microfabricated surface ion trap on a high-finesse optical mirror Opt. Lett. 36 3045-7

[190] Kollath C, Köhl M and Giamarchi T 2007 Scanning tunneling microscopy for ultracold atoms Phys. Rev. A 76063602

[191] Grimm R, Weidemüller M and Ovchinnikov Y B 2000 Optical dipole traps for neutral atoms Adv. At. Mol. Opt. Phys. 42 95-170

[192] Zipkes C, Palzer S, Sias C and Köhl M 2010 A trapped single ion inside a Bose-Einstein condensate Nature 464 388-91

[193] Leibrandt D R, Labaziewicz J, Vuletić V and Chuang I L 2009 Cavity sideband cooling of a single trapped ion Phys. Rev. Lett. 103103001

[194] Rehbein N et al 2007 Optical quenching of metastable magnesium Phys. Rev. A 76043406

[195] James D F V 1998 Quantum dynamics of cold trapped ions with application to quantum computation Appl. Phys. B 66 181-90

[196] Abramowitz M and Stegun I A (ed) 1972 Handbook of Mathematical Functions with Formulas, Graphs, and Mathematical Tables (Washington, DC: GPO)

[197] Magnus W 1954 On the exponential solution of differential equations for a linear operator Commun. Pure Appl. Math. 7 649-73

[198] Blanes S, Casas F, Oteo J A and Ros J 2009 The Magnus expansion and some of its applications Phys. Rep. 470 151-238

[199] Schmid S, Härter A and Hecker Denschlag J 2010 Dynamics of a cold trapped ion in a Bose-Einstein condensate Phys. Rev. Lett. 105133202 